\title{
SPECTRAL INVARIANTS, ANALYSIS OF THE FLOER MODULI SPACE AND GEOMETRY OF THE HAMILTONIAN DIFFEOMORPHISM GROUP
}

\author{
YONG-GEUn OH ${ }^{1}$
}

\begin{abstract}
A BSTRACT. In this paper, we apply spectral invariants, constructed in $[\mathrm{Oh} 5,8]$, to the study of Hamiltonian diffeomorphisms of closed symplectic manifolds $(M, \omega)$. Using spectral invariants, we first construct an invariant norm called the spectral norm on the Hamiltonian diffeomorphism group and obtain several lower bounds for the spectral norm in terms of the $\epsilon$-regularity theorem and the symplectic area of certain pseudo-holomorphic curves. We then apply spectral invariants to the study of length minimizing properties of certain Hamiltonian paths among all paths. In addition to the construction of spectral invariants, these applications rely heavily on the chain level Floer theory and on some existence theorems with energy bounds of pseudoholomorphic sections of certain Hamiltonian fibrations with prescribed monodromy. The existence scheme that we develop in this paper in turn relies on some careful geometric analysis involving adiabatic degeneration and thick-thin decomposition of the Floer moduli spaces which has an independent interest of its own.

We assume that $(M, \omega)$ is strongly semi-positive throughout, which will be removed in a sequel.
\end{abstract}

MSC-class: 53D35, 53D40

\section{Contents}

$\S 1$. Introduction and statement of the main results

$\S 2$. Preliminaries

2.1. The action functional and the action spectrum

2.2. Filtered Floer homology

2.3. Spectral invariants and Floer fundamental cycles

2.4. Comparison of two Cauchy-Riemann equations

PART I: APPLICATIONS OF SPECTRAL INVARIANTS

$\S 3$. Definition of the spectral norm

$\S 4$. The $\epsilon$-regularity theorem and its consequences

Key words and phrases. Hamiltonian diffeomorphism group, invariant norm, spectral invariants, $\epsilon$-regularity theorem, pants product, Hamiltonian fibrations, pseudo-holomorphic sections, Floer fundamental cycles, adiabatic degeneration, thick-thin decomposition.

${ }^{1}$ Partially supported by the NSF Grant \# DMS-9971446 \& DMS-0203593, by \# DMS-9729992 in the Institute for Advanced Study, by the Vilas Associate Award of the University of Wisconsin and by a grant of the Korean Young Scientist Prize 
$\S 5$. Proof of nondegeneracy

$\S 6$. The spectral norm, the ball area and the homological area of $\phi$

$\S 7$. The homological area and the generating function of $\phi$

\section{PART II: ADIABATIC DEGENERATION AND THICK-THIN DECOMPOSITION}

$\S 8$. Proof of the fundamental existence theorem

8.1. Pants products and Hamiltonian fibrations

8.2. Heuristic discussion of the proof of Theorem 5.4

8.3. Construction of solutions I: analysis of thick parts

8.4. Construction of solutions II: analysis of thin parts

8.5. Construction of solutions III: wrap-up

$\S 9$. Local Floer complex and its homology

9.1. The thin part of the Floer boundary operator

9.2. The thin part of the pants product

9.3. The canonical Floer fundamental cycle

9.4. Proof of Theorem 7.1

$\S 10$. Thick-thin decomposition of the Floer moduli space Appendix : Construction of flat connections

\section{$\S 1$. Introduction and the statement of the main results}

This is a sequel to the papers $[\mathrm{Oh} 5,8]$ in which we constructed some invariants called spectral invariants of (time dependent) Hamiltonian functions or of associated Hamiltonian paths. In the present paper we provide several applications of these spectral invariants to the study of the geometry of Hamiltonian diffeomorphisms.

We start by reviewing the definition of the Hofer norm $\|\phi\|$ of a Hamiltonian diffeomorphism $\phi$. For a given Hamiltonian diffeomorphism $\phi \in \mathcal{H} a m(M, \omega)$, we write $F \mapsto \phi$ if the (time-dependent) Hamiltonian function $F:[0,1] \times M \rightarrow \mathbb{R}$ satisfies $\phi_{F}^{1}=\phi$. We will always consider a normalized Hamiltonian $F$, that is satisfying $\int_{M} F_{t} d \mu=0$. The composed flow $t \mapsto \phi_{F}^{t} \circ \phi_{H}^{t}$ is generated by the product Hamiltonian $F \# H$

$$
F \# H(t, x)=F(t, x)+H\left(t,\left(\phi_{F}^{t}\right)^{-1}(x)\right)
$$

and the inverse flow $t \mapsto\left(\phi_{H}^{t}\right)^{-1}$ is generated by the inverse Hamiltonian $\bar{H}$

$$
\bar{H}(t, x)=-H\left(t, \phi_{H}^{t}(x)\right) .
$$

We recall the following standard definitions :

$$
\begin{aligned}
E^{-}(H) & =\int_{0}^{1}-\min H_{t} d t, \quad E^{+}(H)=\int_{0}^{1} \max H_{t} d t \\
\|H\| & =E^{-}(H)+E^{+}(H) .
\end{aligned}
$$


The Hofer norm $\|\phi\|$ is defined by

$$
\|\phi\|=\inf _{H \mapsto \phi}\|H\| .
$$

Noting that $E^{+}(H)=E^{-}(\bar{H}),\|H\|$ and $\|\phi\|$ can be rewritten as

$$
\begin{aligned}
\|H\| & =E^{-}(H)+E^{-}(\bar{H}) \\
\|\phi\| & =\inf _{H \mapsto \phi}\left(E^{-}(H)+E^{-}(\bar{H})\right) .
\end{aligned}
$$

Next we recall the basic properties of the particular spectral invariant $\rho(H ; 1)$ corresponding to the quantum cohomology class $1 \in Q H^{*}(M)$. First, $\rho(H ; 1)$ satisfies the general inequality

$$
-E^{+}(H) \leq \rho(H ; 1) \leq E^{-}(H)
$$

(see Theorem 3.1), and a fortiori the weaker inequality

$$
-\|H\| \leq \gamma(H) \leq\|H\|
$$

for the function $\gamma: C_{m}^{\infty}([0,1] \times M) \rightarrow \mathbb{R}$ defined by

$$
\gamma(H)=\rho(H ; 1)+\rho(\bar{H} ; 1) .
$$

A rather interesting stronger lower bound of $\gamma$ comes from the general triangle inequality

$$
\rho(H ; a)+\rho(F ; b) \geq \rho(H \# F ; a \cdot b)
$$

for spectral invariants where $a \cdot b$ is the quantum product of $a, b \in Q H^{*}(M)$ : Restricting to $a=b=1$, and using the identity $H \# \bar{H}=\underline{0}$ and the normalization axiom $\rho(\underline{0} ; 1)=0$, we derive

$$
\gamma(H)=\rho(H ; 1)+\rho(\bar{H} ; 1) \geq \rho(\underline{0} ; 1)=0 .
$$

Here $\underline{0}$ denotes the zero function.

This leads us to the definition of a non-negative function $\gamma: \operatorname{Ham}(M, \omega) \rightarrow \mathbb{R}_{+}$ defined by

$$
\gamma(\phi):=\inf _{H \mapsto \phi}(\rho(H ; 1)+\rho(\bar{H} ; 1)) .
$$

We would like to point out the similarity between the expressions (1.5) and (1.10). However it should be noted that the nonnegativity of (1.5) is automatic while the nonnegativity of (1.10) is a nontrivial consequence of the triangle inequality (1.9), which is closely tied to the positivity phenomenon in symplectic topology manifested through pseudoholomorphic curves.

Throughout the paper, we will assume the spectrality axiom

$$
\rho(H ; 1) \in \operatorname{Spec}(H)
$$

for nondegenerate Hamiltonians $H$. This then implies the homotopy invariance of $H \mapsto \rho(H ; 1)$ which is in turn needed to prove Lemma $5.2(5.5)$. The spectrality axiom of $\rho(H ; a)$ for arbitrary $H$ and $a$ was proved in the rational $(M, \omega)$ in [Oh8], and in the irrational $(M, \omega)$ in the preprint (math.SG/0406449) (for nondegenerate Hamiltonians). Therefore this assumption is likely to be superfluous. However, until the proof of the spectrality axiom for the irrational case in the preprint math.SG/0406449) is confirmed, we will assume that $(M, \omega)$ is rational in the present paper.

The following theorem provides the general properties of $\gamma: \operatorname{Ham}(M, \omega) \rightarrow \mathbb{R}_{+}$. 
Theorem I. $\gamma: \operatorname{Ham}(M, \omega) \rightarrow \mathbb{R}_{+}$satisfies the following properties :

(1) $\phi=i d$ if and only if $\gamma(\phi)=0$

(2) $\gamma\left(\eta \phi \eta^{-1}\right)=\gamma(\phi)$ for any symplectic diffeomorphism $\eta$

(3) $\gamma(\psi \phi) \leq \gamma(\psi)+\gamma(\phi)$

(4) $\gamma\left(\phi^{-1}\right)=\gamma(\phi)$

(5) $\gamma(\phi) \leq\|\phi\|$.

In particular, $\gamma$ defines a symmetric (i.e., $\gamma(\phi)=\gamma\left(\phi^{-1}\right)$ ) invariant norm on $\operatorname{Ham}(M, \omega)$.

All but (1), nondegeneracy of $\gamma$, are immediate consequences of the general properties of $\rho(H ; 1)$.

A similar construction was previously carried out by Viterbo $[\mathrm{V}]$ and by the author $[\mathrm{Oh} 4]$ for the cotangent bundle considering Hamiltonian deformations of the zero section, and by Schwarz [Sc] for Hamiltonian diffeomorphisms on the (symplectically) aspherical symplectic manifold $(M, \omega)$. In all these cases, $\gamma$ was defined by

$$
\gamma(H)=\rho(H ; 1)-\rho(H ; \mu)
$$

$\mu$ being the volume class in $H^{*}(M)$. The quantity (1.12) coincides with the expression $(1.8)$ in the aspherical $(M, \omega)$ because in that case we have the additional identity

$$
\rho(\bar{H} ; 1)=-\rho(H ; \mu)
$$

[V], [Sc]. But Polterovich observed [Po2] that this latter identity fails in the nonexact case due to the quantum contributions. It turns out that for the general case the right way of defining an invariant norm satisfying the triangle inequality is (1.8) and (1.10), not (1.12). As illustrated by Ostrover [Os] in the aspherical case, $\gamma$ is different from Hofer's norm.

In a way, our nondegeneracy proof for $\gamma$ is a combination of the techniques Hofer [Ho] and Lalonde-McDuff [LM] used to prove nondegeneracy of Hofer's norm. Recall Hofer used the classical critical point theory argument in his proof for $\mathbb{C}^{n}$ while Lalonde-McDuff used the method of pseudoholomorphic curves via general Nonsqueezing Theorem. What we present is a more sophisticated critical point theory for general $(M, \omega)$ via Floer theory, which is also naturally tied to pseudoholomorphic curves and quantum cohomology through deformations of Hamiltonian functions. However for the lower estimate of $\gamma(\phi)$, instead of the ball area of $\phi$ (see Definition 4.1), we use other geometric invariants of $\phi$ which naturally arise in our chain level arguments and better suits Floer theory. These invariants, denoted by $A(\phi)$ and $A(\phi ; 1)$ respectively, depend only on $\phi$ itself, independently of its generating Hamiltonian. Our definition of $A(\phi)$ resembles that of a similar invariant used by the author in $[\mathrm{Oh} 2,3]$ and Chekanov $[\mathrm{Ch}]$ in the context of Lagrangian submanifolds.

Now we will provide a precise description of the analytic invariant $A(\phi)$ here in the introduction partly because it is needed to explain other main results of the present paper. It also has a second purpose : we would like to emphasize the precise details needed in various constructions of the Floer theory and spectral invariants which are essential for the applications studied in the present paper. These details have not been emphasized enough in the literature but we believe that they deserve more attention and scrutiny in the future. 
Let $\phi$ be a Hamiltonian diffeomorphism that has only a finite number of fixed points (e.g., non-degenerate ones). We denote by $J_{0}$ a compatible almost complex structure on $(M, \omega)$ and by $\mathcal{J}_{\omega}$ the set of all such structures. For a given pair $\left(\phi, J_{0}\right)$, we consider paths $J^{\prime}:[0,1] \rightarrow \mathcal{J}_{\omega}$ with

$$
J^{\prime}(0)=J_{0}, \quad J^{\prime}(1)=\phi^{*} J(0)
$$

and denote the set of such paths by

$$
j_{\left(\phi, J_{0}\right)} .
$$

One can extend this family to $\mathbb{R}$ by the equation

$$
J^{\prime}(t+1)=\phi^{*} J^{\prime}(t) .
$$

For each given $J^{\prime} \in j_{\left(\phi, J_{0}\right)}$, we define the constants

$$
\begin{aligned}
A_{S}\left(\phi, J_{0} ; J^{\prime}\right)=\inf \left\{\omega([u]) \mid u: S^{2} \rightarrow M\right. \text { non-constant and } \\
\text { satisfying } \left.\bar{\partial}_{J_{t}^{\prime}} u=0 \text { for some } t \in[0,1]\right\}
\end{aligned}
$$

and

$$
A_{S}\left(\phi, J_{0}\right)=\sup _{J^{\prime} \in j_{\left(\phi, J_{0}\right)}} A_{S}\left(\phi, J_{0} ; J^{\prime}\right) .
$$

As usual, we set $A_{S}\left(\phi, J_{0} ; J^{\prime}\right)=\infty$ if there is no $J_{t}^{\prime}$-holomorphic sphere for any $t \in[0,1]$ e.g., as in the weakly exact case. The positivity of $A_{S}\left(\phi, J_{0} ; J^{\prime}\right)$ and so $A_{S}\left(\phi, J_{0}\right)$ is a consequence of the one parameter version of the uniform $\epsilon$-regularity theorem (see [SU], [Oh1]). We give the proof of positivity in section 4.

Next for each given $J^{\prime} \in j_{\left(\phi, J_{0}\right)}$, we consider the equation

$$
\left\{\begin{array}{l}
\frac{\partial v}{\partial \tau}+J_{t}^{\prime} \frac{\partial v}{\partial t}=0 \\
\phi(v(\tau, 1))=v(\tau, 0), \quad \int_{\mathbb{R} \times[0,1]}\left|\frac{\partial v}{\partial \tau}\right|_{J_{t}^{\prime}}^{2}<\infty
\end{array}\right.
$$

in $v: \mathbb{R} \times[0,1] \rightarrow M$. Here the norm $|\cdot|_{J_{t}^{\prime}}$ is the norm induced by the Riemannian metric

$$
g_{J_{t}^{\prime}}:=\omega\left(\cdot, J_{t}^{\prime} \cdot\right)
$$

The choice (1.13) of $J^{\prime}$ enables us to interpret solutions of (1.14) as pseudo-holomorphic sections of the mapping cylinder of $\phi$ with respect to a suitably chosen almost complex structure on the mapping cylinder. See subsection 2.3 for a more detailed discussion of this.

We now define the constant

$$
A_{D}\left(\phi, J_{0} ; J^{\prime}\right):=\inf _{v}\left\{\int v^{*} \omega, \mid v \text { non-constant solution of }(1.14)\right\}
$$

for each $J \in j_{\left(\phi, J_{0}\right)}$. In section 4, we will prove that if $\phi$ is nondegenerate, $A_{D}\left(\phi, J_{0} ; J^{\prime}\right)>0$. Combining $A_{S}\left(\phi, J_{0} ; J^{\prime}\right)$ and $A_{D}\left(\phi, J_{0} ; J^{\prime}\right)$, we define

$$
A\left(\phi, J_{0} ; J^{\prime}\right)=\min \left\{A_{S}\left(\phi, J_{0} ; J^{\prime}\right), A_{D}\left(\phi, J_{0} ; J^{\prime}\right)\right\} .
$$


Finally we set

$$
A\left(\phi, J_{0}\right):=\sup _{J^{\prime} \in j_{\left(\phi, J_{0}\right)}} A\left(\phi, J_{0} ; J^{\prime}\right)
$$

and

$$
A(\phi)=\sup _{J_{0} \in \mathcal{J}_{\omega}} A\left(\phi, J_{0}\right) .
$$

Note that when $(M, \omega)$ is weakly exact, in which case $A_{S}\left(\phi, J_{0} ; J^{\prime}\right)=\infty, A\left(\phi, J_{0}\right)$ is reduced to

$$
A\left(\phi, J_{0}\right)=\sup _{J^{\prime} \in j_{\left(\phi, J_{0}\right)}}\left\{A_{D}\left(\phi, J_{0} ; J^{\prime}\right)\right\} .
$$

It is now clear from the definition of $A\left(\phi, J_{0}\right)$ that $A\left(\phi, J_{0}\right)>0$ and so we have $A(\phi)>0$. At this moment, however, we note that a priori $A\left(\phi, J_{0}\right)$ (and so $A(\phi)$ ) could be $+\infty$.

In section 5 , we prove the following inequality, which on the one hand gives rise to nondegeneracy of $\gamma$ and on the other hand proves $A(\phi)<\infty$.

Theorem II. Let $\phi$ be nondegenerate and $A(\phi)$ be the constant defined in (1.15). Then we have

$$
\gamma(\phi) \geq A(\phi)
$$

In particular $A(\phi)$ is finite and $\gamma(\phi)>0$.

A priori, Theorem II does not guarantee positivity of $\gamma(\phi)$ for degenerate $\phi$ : Recall that for the definition of $A(\phi)$ we assumed that $\phi$ is nondegenerate. However Theorem I (2) implies that the null-set

$$
\operatorname{null}(\gamma)=\{\phi \in \operatorname{Ham}(M, \omega) \mid \gamma(\phi)=0\}
$$

is a normal subgroup of $\operatorname{Ham}(M, \omega)$. Theorem II shows that null $(\gamma)$ is a proper subgroup of $\operatorname{Ham}(M, \omega)$ and so it must be a trivial subgroup thereof by Banyaga's theorem on the simpleness of $\operatorname{Ham}(M, \omega)$ [Ba]. This proves the nondegeneracy of $\gamma$.

Exploiting the fact that the definition of $\gamma$ involves only the identity class $1 \in$ $Q H^{*}(M)$, we refine the definition of $A(\phi)$ to get a stronger invariant denoted by $A(\phi ; 1)$, which we call the homological area of $\phi . A(\phi ; 1)$ always satisfies $A(\phi ; 1) \geq$ $A(\phi)$ and is more computable than $A(\phi)$. Referring to section 6 for the precise definition of $A(\phi ; 1)$, we state

Theorem III. For any nondegenerate Hamiltonian diffeomorphism $\phi$, we have

$$
\gamma(\phi) \geq A(\phi ; 1) .
$$

To provide some intuitive meaning to $A(\phi ; 1)$, we provide one corollary of Theorem III which is an immediate translation of the inequality $A(\phi ; 1) \leq \gamma(\phi)$ from the definition of $A(\phi ; 1)$.

Corollary. Let $\phi$ be nondegenerate and let $q \in M \backslash$ Fix $\phi$. Then for any choice of $J_{0} \in \mathcal{J}_{\omega}$ and $J^{\prime} \in j_{\left(\phi, J_{0}\right)}$, one of the following alternative holds:

(1) there exists a non-constant $J_{t}^{\prime}$-holomorphic sphere passing through $q$ for some $t \in[0,1]$ and of area less than or equal to $\gamma(\phi)$

(2) (1.14) has a non-constant solution passing through $q$ and of area less than or equal to $\gamma(\phi)$. 
The inequality $A(\phi) \leq \gamma(\phi)$ in Theorem II also implies a similar existence result but without the phrase "passing through $q$ " which illustrates the fact that the definition of $A(\phi ; 1)$ is tied to the fundamental class $[M]=1^{b}$. Therefore $A(\phi ; 1)$ is a much better invariant than $A(\phi)$ for a nondegenerate $\phi$. In fact when a sequence $\left\{\phi_{i}\right\}$ of nondegenerate Hamiltonian diffeomorphisms converges to, say, a degenerate $\phi \neq i d$ in the $C^{0}$-topology, $A\left(\phi_{i} ; 1\right)$ may fail to converge to zero together with $A\left(\phi_{i}\right)$. In section 6 , we will provide an example of a Hamiltonian diffeomorphism $\phi$ on $S^{2}$ for which $0<A(\phi)<A(\phi ; 1)$ and also construct a sequence of nondegenerate $\phi_{i}$ for which $A\left(\phi_{i}\right)$ converges to 0 but $A\left(\phi_{i} ; 1\right)$ remains constant. We conjecture in general that $A(\phi ; 1)$ is continuous in $C^{\infty}$-topology (maybe even in $C^{0}$-topology) and so can be extended to arbitrary smooth Hamiltonian diffeomorphisms.

Noting $\|\phi\| \geq \gamma(\phi)$, we also derive the following corollary.

Corollary. The Hofer norm $\|\phi\|$ satisfies

$$
\|\phi\| \geq A(\phi ; 1) .
$$

Using the spectral norm $\gamma(\phi)$ and the homological area $A(\phi ; 1)$ of $\phi$, both of which depend only on $\phi$ and not on its generating Hamiltonian function, we study the length minimizing property of certain Hamiltonian paths among all paths with their end points at the identity and $\phi$. For simplicity of exposition, we will restrict to the case where $\phi$ is $C^{1}$-close to the identity in this paper, leaving the proof for the more general engulfable Hamiltonian diffeomorphisms to a sequel [Oh10].

For the $C^{1}$-small case (or more generally for the engulfable case), we can represent the subset $\operatorname{graph}(\phi) \subset(M,-\omega) \times(M, \omega)$ in the following form

$$
\Phi(\operatorname{graph} \phi)=\operatorname{graph} d S_{\phi} .
$$

where $\Phi: \mathcal{U} \subset M \times M \rightarrow \mathcal{V} \subset T^{*} \Delta$ is a Darboux-Weinstein chart of the diagonal Lagrangian submanifold $\Delta \subset(M-\omega) \times(M, \omega)$. The function $S_{\phi}$ is unique up to the addition of a constant and is called the generating function of $\phi$. For a function $f: M \rightarrow \mathbb{R}$, we define

$$
\operatorname{osc}(f)=\max f-\min f .
$$

Theorem IV. Suppose that $\phi$ is sufficiently $C^{1}$-small. Then we have

$$
\gamma(\phi)=A(\phi ; 1)=\operatorname{osc}\left(S_{\phi}\right)=\|\phi\| .
$$

An immediate corollary of this theorem is the following result by McDuff [Mc].

Corollary [Proposition 1.8, Mc]. Let $\phi$ be sufficiently $C^{1}$-small and let $\phi^{t}$ be the Hamiltonian path determined by the equation

$$
\Phi\left(\operatorname{graph} \phi^{t}\right)=\operatorname{graph} t d S_{\phi} .
$$

Then the path $t \in[0,1] \mapsto \phi^{t} \in \operatorname{Ham}(M, \omega)$ is a Hamiltonian path which is length minimizing among all paths from the identity to $\phi$.

The proof of Theorem IV, more specifically the inequality $A(\phi ; 1) \geq \operatorname{osc}\left(S_{\phi}\right)$, is also a consequence of an existence theorem for the equation (1.14) with a suitably chosen asymptotic condition. Besides Theorem 5.4 this existence proof heavily 
relies on the thick-thin decomposition of the Floer moduli space. Such a decomposition exists when $H$ is sufficiently $C^{2}$-small. Study of the persistence of such a decomposition will enable us to extend Theorem IV to the cases where $\phi$ is not necessarily $C^{1}$-small. We will study further properties of the homological area $A(\phi ; 1)$ and the relevance of $A(\phi ; 1)$ to the energy-capacity inequality elsewhere.

Now the organization of the paper is in order. In section 2 , we briefly review various points needed throughout the paper about chain level Floer theory and spectral invariants of Hamiltonian diffeomorphisms. We also set up our conventions concerning the definition of the action functional and the gradings of quantum cohomology and Floer homology. We then carefully review two different set-ups of Floer homology from the literature as well as the correspondences between them : one is the dynamical version of perturbed Cauchy-Riemann equations [Fl3] and the other is the mapping cylinder version [Fl1, DS]. A careful study of this correspondence is one of the crucial elements in our construction and for the proof of nondegeneracy of the norm $\gamma$. A similar correspondence was used by the author in $[\mathrm{Oh} 3,4]$ in the context of the 'open string' version of Floer homology i.e., in the context of Lagrangian submanifolds.

After this review the paper is divided into two parts. Part I, consisting of sections $3-7$, contains the main applications of spectral invariants mentioned above. Part II, consisting of sections 8 - 10, contains key analysis needed in the proofs of the main theorems stated in part I. Section 8 contains the proof of Theorem 5.4, which relies on the analysis of adiabatic degeneration in subsection 8.4. In relation to the Fukaya category of the cotangent bundle, similar analysis, but without quantum contributions, was carried out in [FOh] in the context of degeneration of the moduli space of pseudoholomorphic discs to the moduli space of graph flows. The proof of the fundamental existence theorem involves a rather delicate geometric analysis that is not common in the literature, especially in the literature of symplectic geometry. As far as we know, [FOh] is the only other paper in the literature of symplectic geometry which deals with analysis of adiabatic degeneration in details. The paper [DS] by Dostoglou and Salamon studies a different kind of adiabatic degeneration in relation to the Atiyah-Floer conjecture, whose nature is somewhat different from ours. In addition to this degeneration analysis, proof of the lower estimate of $\gamma(\phi)$ in Theorem III relies also on the chain level argument of the Floer theory that is developed in [Oh5].

Sections 9 and 10 give the proof of thick-thin decomposition of the Floer moduli spaces needed for the proof of Theorem IV. This is the analog to the similar decomposition result used by the author [Oh2] in the study of the Maslov class obstruction to the Lagrangian embeddings via the Floer homology of Lagrangian submanifolds. The analysis developed in part II have other applications which will be presented elsewhere. The appendix provides an explicit construction of a flat connection for the triple $(H, \widetilde{H} \# \epsilon f ; \epsilon f)$ of Hamiltonians that is used in section 8 .

To make the main stream of ideas transparent in this paper without getting bogged down with technicalities related to transversality issues of various moduli spaces, we will assume in the present paper that $(M, \omega)$ is strongly semi-positive in the sense of [Se] and [En1]. A closed symplectic manifold is called strongly semi-positive if there is no spherical homology class $A \in \pi_{2}(M)$ such that

$$
\omega(A)>0, \quad 2-n \leq c_{1}(A)<0 .
$$

Under this condition the transversality problem concerning various moduli spaces of 
pseudo-holomorphic curves is standard. We will not mention this generic transversality question at all in the main body of the paper unless it is absolutely necessary. The scheme of the proof should go through on arbitrary closed symplectic manifolds in the general framework of virtual moduli cycles. In a sequel to the present paper we will incorporate this enhanced machinery to generalize all the results to arbitrary closed symplectic manifolds.

For those who are familiar with the construction of spectral invariants detailed in $[\mathrm{Oh} 8]$ and want to get only the idea behind the nondegeneracy proof for $\gamma$, we recommend them to read section 5 and the heuristic discussion in subsection 8.2. The nondegeneracy proof is based on the existence result of certain pseudoholomorphic curves as used in [Oh4] : In [Oh4] the author uses the existence result to prove positivity of the quantity (1.12) when $\phi_{H}^{1} \neq i d$ on the cotangent bundle $T^{*} N$. To prove this existence theorem in [Oh4], the author uses the cap action realized by the degenerate pants in the context of Morse homology and then proves that the cap action isomorphism $\cap \mu: H_{n}(N) \rightarrow H_{0}(N)$ coincides with the standard pants product $1 * \mu=\mu$ in the Floer homology after applying the Poincaré duality. The author's derivation of the latter fact in turn is based on the analytical result from $[\mathrm{FOh}]$ mentioned above. In the present paper, we use the nontrivial quantum product relation ' $1 * 1=1$ ' instead which suits better our definition (1.10) of $\gamma$ and also produces such geometric lower bounds as those obtained in Theorem III and IV, in addition to the nondegeneracy result for $\gamma$.

The main results of the present work were presented in our paper entitled "Minimax theory, spectral invariants and geometry of the Hamiltonian diffeomorphism group" (ArXiv:math.SG/0206092), containing fewer details and some minor errors, which has circulated since June, 2002. We isolate and expand the applications of the spectral invariants in this paper with more clarification and corrections of some details, leaving the construction of them to a separate paper [Oh8]. Other applications of the spectral invariants to the study of length minimizing properties of Hamiltonian paths are given by the author [Oh7].

During the submission of the present paper, there appeared a book by McDuff and Salamon ("J-holomorphic Curves and Symplectic Topology", AMS, Providence, 2004) in which the authors present a description of the spectral invariants and a nondegeneracy proof in the rational case based on the approach relying on the so called 'PSS-isomorphism' [PSS]. As we indicated in the above mentioned preprint and in [Oh8], such an approach should be possible eventually. However, to make the approach well-founded, it remains to write out a proof of the isomorphism property of the PSS-map which is used in various constructions carried out in [PSS] and in the book mentioned above.

We thank the Institute for Advanced Study for the excellent environment and hospitality during our participation in the 2001-2002 program "Symplectic Geometry and Holomorphic Curves". Much of the present work was finished during our stay in IAS. We thank D. McDuff for useful communications at IAS. The final writing of the previous version of the paper was carried out while visiting the Korea Institute for Advanced Study in Seoul. We thank KIAS for providing an uninterrupted quiet environment and time for writing the paper and an excellent atmosphere for research.

Thanks to M. Entov and L. Polterovich for enlightening discussions on spectral invariants and for explaining their applications [En2], [EnP] of spectral invariants to study of the Hamiltonian diffeomorphism group, and Y. Ostrover for explaining 
his work from [Os] to us during our visit to Tel-Aviv University. We also thank P. Biran and L. Polterovich for their invitation to Tel-Aviv University and hospitality during our visit. We are very much indebted to the referees for their careful reading of the previous versions of the paper and for many helpful questions, corrections and suggestions, which have both reinforced the content of the paper and led to much improvement in the presentation and readability of the paper. We also thank P. Spaeth for his careful proofreading of the previous version of the paper and many helpful comments in the presentation and in the English of the paper, and N. Kieserman for the final touch in English of the introduction of the paper.

\section{$\S 2$. Preliminaries}

\subsection{The action functional and the action spectrum}

Let $(M, \omega)$ be any closed symplectic manifold and $\Omega_{0}(M)$ be the set of contractible loops. We first recall the construction from $[\mathrm{HoS}]$ of a covering space of $\Omega_{0}(M)$, which we denote by $\widetilde{\Omega}_{0}(M)$.

Let $(\gamma, w)$ be a pair consisting of $\gamma \in \Omega_{0}(M)$ and a disc $w$ bounding $\gamma$. We say that $(\gamma, w)$ is $\Gamma$-equivalent to $\left(\gamma, w^{\prime}\right)$ if

$$
\omega\left(\left[w^{\prime} \# \bar{w}\right]\right)=0 \quad \text { and } \quad c_{1}\left(\left[w^{\prime} \# \bar{w}\right]\right)=0
$$

where $\bar{w}=w \circ c, c: D^{2} \rightarrow D^{2}$ is the complex conjugation and $w^{\prime} \# \bar{w}$ is the obvious glued sphere. Here $\Gamma$ denotes the group

$$
\Gamma=\frac{\pi_{2}(M)}{\operatorname{ker}\left(\left.\omega\right|_{\pi_{2}(M)}\right) \cap \operatorname{ker}\left(\left.c_{1}\right|_{\pi_{2}(M)}\right)} .
$$

We denote by $[\gamma, w]$ the $\Gamma$-equivalence class of $(\gamma, w)$ and by $\pi: \widetilde{\Omega}_{0}(M) \rightarrow \Omega_{0}(M)$ the canonical projection. We also call $\widetilde{\Omega}_{0}(M)$ the $\Gamma$-covering space of $\Omega_{0}(M)$. The period group of $(M, \omega)$ is defined by

$$
\Gamma_{\omega}:=\left\{\omega(A) \mid A \in \pi_{2}(M)\right\}=\omega(\Gamma) \subset \mathbb{R} .
$$

It is either a discrete or a countable dense subset of $\mathbb{R}$.

Unless otherwise stated, we will always consider one-periodic Hamiltonian functions $H:[0,1] \times M \rightarrow \mathbb{R}$ normalized by

$$
\int_{M} H_{t} d \mu=0
$$

where $d \mu$ is the Liouville measure of $(M, \omega)$. We denote by

$$
C_{m}^{\infty}([0,1] \times M)
$$

the set of such Hamiltonian functions, where " $m$ " stands for the word "mean zero".

When a periodic normalized Hamiltonian $H:(\mathbb{R} / \mathbb{Z}) \times M \rightarrow \mathbb{R}$ is given, we define the action functional $\mathcal{A}_{H}: \widetilde{\Omega}_{0}(M) \rightarrow \mathbb{R}$ by

$$
\mathcal{A}_{H}([\gamma, w])=-\int w^{*} \omega-\int H(t, \gamma(t)) d t .
$$

We denote by $\operatorname{Per}(H)$ the set of periodic orbits of $X_{H}$. 
Definition 2.1. We define the action spectrum of $H$, denoted as $\operatorname{Spec}(H) \subset \mathbb{R}$, by

$$
\operatorname{Spec}(H):=\left\{\mathcal{A}_{H}(z, w) \in \mathbb{R} \mid[z, w] \in \widetilde{\Omega}_{0}(M), z \in \operatorname{Per}(H)\right\}
$$

i.e., the set of critical values of $\mathcal{A}_{H}: \widetilde{\Omega}(M) \rightarrow \mathbb{R}$.

The following was proven in [Oh5].

Proposition 2.2. $\operatorname{Spec}(H)$ is a measure zero subset of $\mathbb{R}$ for any $H$.

We say that two Hamiltonians $F$ and $G$ are equivalent and denote $F \sim G$ if they are connected by a one-parameter family of Hamiltonians $\left\{F^{s}\right\}_{0 \leq s \leq 1}$ such that $F^{s} \mapsto \phi$ for all $s \in[0,1]$. We write $[F]$ or $[\phi, F]$ for the equivalence class of $F$. Under the canonical one-one correspondence between $H$ and its Hamiltonian path $\phi_{F}: t \mapsto \phi_{F}^{t},[F]$ corresponds to the path-homotopy class of the Hamiltonian path $\phi_{H}:[0,1] \rightarrow \operatorname{Ham}(M, \omega)$ with fixed end points. If we denote by $\widetilde{\operatorname{Ham}}(M, \omega)$ the set of path-homotopy classes of the based path at the identity of $\operatorname{Ham}(M, \omega)$, then the set of $[F]$ coincides with $\widetilde{\operatorname{Ham}}(M, \omega)$.

The following lemma was proven in the aspherical case in [Sc, Po1]. We refer the reader to [Oh6] for complete details of its proof in the general case.

Proposition 2.3. If $F \sim G$, we have

$$
\operatorname{Spec}(F)=\operatorname{Spec}(G)
$$

as a subset of $\mathbb{R}$.

Definition 2.4. For given $h \in \widetilde{\operatorname{Ham}}(M, \omega)$, we define

$$
\operatorname{Spec}(h)=\operatorname{Spec}(H)
$$

for a (and so any) $H$ with $[H]=h$.

\subsection{Filtered Floer homology}

Suppose that $\phi \in \operatorname{Ham}(M, \omega)$ is nondegenerate in the sense of Lefshetz fixed point theory: the derivative $T_{p} \phi: T_{p} M \rightarrow T_{p} M$ has no eigenvalue one at each fixed point $p \in M$. We call a Hamiltonian $H: S^{1} \times M \rightarrow \mathbb{R}$ nondegenerate if $\phi_{H}^{1}=\phi$ is nondegenerate Hamiltonian diffeomorphism.

For each nondegenerate $H: S^{1} \times M \rightarrow \mathbb{R}$, we consider the free $\mathbb{Q}$ vector space over

$$
\operatorname{Crit} \mathcal{A}_{H}=\left\{[z, w] \in \widetilde{\Omega}_{0}(M) \mid z \in \operatorname{Per}(H)\right\} .
$$

To be able to define the Floer boundary operator correctly, we need to complete this vector space downward with respect to the real filtration provided by the action $\mathcal{A}_{H}([z, w])$ of the element $[z, w]$ of $(2.6)$. More precisely, as in $[\mathrm{Fl} 2, \mathrm{HoS}]$, we give the following definition.

Definition 2.5. Consider the formal sum

$$
\beta=\sum_{[z, w] \in \operatorname{Crit} \mathcal{A}_{H}} a_{[z, w]}[z, w], a_{[z, w]} \in \mathbb{Q}
$$


(1) We call those $[z, w]$ with $a_{[z, w]} \neq 0$ generators of the sum $\beta$ and write

$$
[z, w] \in \beta .
$$

We also say that $[z, w]$ contributes to $\beta$ in that case.

(2) We define the support of $\beta$ by

$$
\operatorname{supp}(\beta):=\left\{[z, w] \in \operatorname{Crit} \mathcal{A}_{H} \mid a_{[z, w]} \neq 0 \text { in the sum }(2.7)\right\} .
$$

(3) We call the formal sum $\beta$ a Novikov Floer chain (or simply a Floer chain) if it satisfies

$$
\#\left(\operatorname{supp}(\beta) \cap\left\{[z, w] \mid \mathcal{A}_{H}([z, w]) \geq \lambda\right\}\right)<\infty
$$

for any $\lambda \in \mathbb{R}$. We denote by $C F_{*}(H)$ the set of Floer chains.

Note that $C F_{*}(H)$ is a graded $\mathbb{Q}$-vector space which is infinite dimensional in general, unless $\pi_{2}(M)=0$. As in [Oh5], we introduce the following notion which is a crucial concept for the mini-max argument that we carry out in this paper.

Definition 2.6. Let $\beta$ be a Floer chain in $C F_{*}(H)$. We define the level of a chain $\beta$ and denote it by

$$
\lambda_{H}(\beta)=\max _{[z, w]}\left\{\mathcal{A}_{H}([z, w]) \mid[z, w] \in \operatorname{supp}(\beta)\right\}
$$

if $\beta \neq 0$, and set $\lambda_{H}(0)=-\infty$ as usual.

We briefly review construction of basic operators in the Floer homology theory [Fl3] and their effects on the level of a given Floer chain.

Let $J=\left\{J_{t}\right\}_{0 \leq t \leq 1}$ be a periodic family of compatible almost complex structures on $(M, \omega)$. For each given pair $(H, J)$, we consider the perturbed Cauchy-Riemann equation

$$
\left\{\begin{array}{l}
\frac{\partial u}{\partial \tau}+J\left(\frac{\partial u}{\partial t}-X_{H}(u)\right)=0 \\
\lim _{\tau \rightarrow-\infty} u(\tau)=z^{-}, \lim _{\tau \rightarrow \infty} u(\tau)=z^{+} .
\end{array}\right.
$$

This equation, when lifted to $\widetilde{\Omega}_{0}(M)$, is the negative gradient flow of $\mathcal{A}_{H}$ with respect to the $L^{2}$-metric on $\widetilde{\Omega}_{0}(M)$ induced by the family of metrics on $M$

$$
g_{J_{t}}=(\cdot, \cdot)_{J_{t}}:=\omega\left(\cdot, J_{t} \cdot\right)
$$

this $L^{2}$-metric is defined by

$$
\langle\xi, \eta\rangle_{J}:=\int_{0}^{1}(\xi, \eta)_{J_{t}} d t
$$

A simple computation then shows that the $L^{2}$-gradient vector field of $\mathcal{A}_{H}$ on $\widetilde{\Omega}_{0}(M)$, denoted by $\operatorname{grad}_{J} \mathcal{A}_{H}$, is given by the formula

$$
\left(\operatorname{grad}_{J} \mathcal{A}_{H}\right)([\gamma, w])=J\left(\dot{\gamma}-X_{H}(\gamma)\right)
$$


from which we also derive that the gradient is projectable to $\Omega_{0}(M)$.

We also denote by $\|v\|_{J_{0}}$ the norm of the vector $v$ with respect to the metric $g_{J_{0}}$, i.e.,

$$
\|v\|_{J_{0}}^{2}=(v, v)_{J_{0}}=\omega\left(v, J_{0} v\right)
$$

for $v \in T M$.

Now suppose that $(H, J)$ is regular in the Floer theoretic sense. For each given $\left[z^{-}, w^{-}\right]$and $\left[z^{+}, w^{+}\right]$, we define the moduli space

$$
\mathcal{M}\left(H, J ;\left[z^{-}, w^{-}\right],\left[z^{+}, w^{+}\right]\right)
$$

to be the set of solutions $u$ of (2.9) having finite energy and satisfying the homotopy condition

$$
w^{-} \# u \sim w^{+} .
$$

As a path in $\widetilde{\Omega}_{0}(M)$, we write the asymptotic condition of $u$ as

$$
u(-\infty)=\left[z^{-}, w^{-}\right], \quad u(\infty)=\left[z^{+}, w^{+}\right] .
$$

The Floer boundary map $\partial=\partial_{(H, J)}: C F_{*}(H) \rightarrow C F_{*}(H)$, when the ConleyZehnder indices of $\left[z^{-}, w^{-}\right]$and $\left[z^{+}, w^{+}\right]$satisfy

$$
\mu_{H}\left(\left[z^{-}, w^{-}\right]\right)-\mu_{H}\left(\left[z^{+}, w^{+}\right]\right)=1,
$$

is defined by its matrix coefficient

$$
\left\langle\partial\left(\left[z^{-}, w^{-}\right]\right),\left[z^{+}, w^{+}\right]\right\rangle:=\#\left(\mathcal{M}\left(H, J ;\left[z^{-}, w^{-}\right],\left[z^{+}, w^{+}\right]\right)\right)
$$

where $\#\left(\mathcal{M}\left(H, J ;\left[z^{-}, w^{-}\right],\left[z^{+}, w^{+}\right]\right)\right)$is the algebraic number of the elements in $\mathcal{M}\left(H, J ;\left[z^{-}, w^{-}\right],\left[z^{+}, w^{+}\right]\right)$, and is set to be zero otherwise.

We refer to subsection 2.4 for more discussion about the energy of the map $u$. The Floer boundary map

$$
\partial=\partial_{(H, J)}: C F_{*}(H) \rightarrow C F_{*}(H)
$$

has degree -1 and satisfies $\partial \circ \partial=0$.

Definition 2.7. We say that a Floer chain $\beta \in C F(H)$ a is Floer cycle if $\partial \beta=0$ and a Floer boundary if $\beta=\partial \delta$ for a Floer chain $\delta$. Two Floer chains $\beta, \beta^{\prime}$ are said to be homologous if $\beta^{\prime}-\beta$ is a boundary.

When we are given a family $(\mathcal{H}, j)$ with $\mathcal{H}=\left\{H^{s}\right\}_{0 \leq s \leq 1}$ and $j=\left\{J^{s}\right\}_{0 \leq s \leq 1}$, the chain homomorphism

$$
h_{(\mathcal{H}, j)}: C F_{*}\left(H^{0}\right) \rightarrow C F_{*}\left(H^{1}\right)
$$

is defined by considering the non-autonomous equation

$$
\left\{\begin{array}{l}
\frac{\partial u}{\partial \tau}+J^{\rho(\tau)}\left(\frac{\partial u}{\partial t}-X_{H^{\rho(\tau)}}(u)\right)=0 \\
\lim _{\tau \rightarrow-\infty} u(\tau)=z^{-}, \lim _{\tau \rightarrow \infty} u(\tau)=z^{+}
\end{array}\right.
$$


with the condition

$$
w^{-} \# u \sim w^{+} .
$$

Here $\rho$ is a cut-off function of the type $\rho: \mathbb{R} \rightarrow[0,1]$,

$$
\begin{aligned}
& \rho(\tau)= \begin{cases}0 & \text { for } \tau \leq-R \\
1 & \text { for } \tau \geq R\end{cases} \\
& \rho^{\prime}(\tau) \geq 0
\end{aligned}
$$

for some $R>0$ (Here the monotonicity of $\rho$ is not essential but provides a better estimate in general). $h_{(\mathcal{H}, j)}$ has degree 0 and satisfies

$$
\partial_{\left(H^{1}, J^{1}\right)} \circ h_{(\mathcal{H}, j)}=h_{(\mathcal{H}, j)} \circ \partial_{\left(H^{0}, J^{0}\right)} .
$$

Two such chain maps $h_{\left(\mathcal{H}^{1}, j^{1}\right)}, h_{\left(\mathcal{H}^{2}, j^{2}\right)}$ are also known to be chain homotopic [Fl3].

The following upper estimate of the action change can be proven by the same argument as that of the proof of [Theorem 7.2, Oh3].

Proposition 2.8. Let $H$ and $K$ be any Hamiltonians, not necessarily nondegenerate, $j=\left\{J^{s}\right\}_{s \in[0,1]}$ be any given homotopy and $\mathcal{H}^{\text {lin }}=\left\{H^{s}\right\}_{0 \leq s \leq 1}$ be the linear homotopy $H^{s}=(1-s) H+s K$. Suppose that (2.12) has a solution with finite energy and satisfies (2.13). Then we have the identity

$$
\begin{aligned}
\mathcal{A}_{K}\left(\left[z^{+}, w^{+}\right]\right) & -\mathcal{A}_{H}\left(\left[z^{-}, w^{-}\right]\right) \\
& =-\int\left|\frac{\partial u}{\partial \tau}\right|_{J^{\rho_{1}(\tau)}}^{2}-\int_{-\infty}^{\infty} \rho^{\prime}(\tau) \int_{0}^{1}(K(t, u(\tau, t))-H(t, u(\tau, t))) d t d \tau \\
& \leq-\int\left|\frac{\partial u}{\partial \tau}\right|_{J^{\rho_{1}(\tau)}}^{2}+\int_{0}^{1}-\min _{x \in M}\left(K_{t}-H_{t}\right) d t \\
& \leq \int_{0}^{1}-\min _{x \in M}\left(K_{t}-H_{t}\right) d t .
\end{aligned}
$$

The following is an immediate corollary of Proposition 2.8.

Corollary 2.9. Let $H$ and $K$ be nondegenerate Hamiltonians, and let

$$
\left[z^{+}, w^{+}\right], \quad\left[z^{-}, w^{-}\right]
$$

be critical points of $\mathcal{A}_{H}$ and $\mathcal{A}_{K}$ respectively. Suppose that there exists a family $\left\{J^{s}\right\}_{0 \leq s \leq 1}$ such that the equation (2.12) for the linear homotopy $\mathcal{H}^{\text {lin }}$ has a solution $u$ satisfying (2.13) and

$$
u(-\infty)=\left[z^{-}, w^{-}\right], \quad u(\infty)=\left[z^{+}, w^{+}\right]
$$

where $u$ is considered as a gradient trajectory on $\widetilde{\Omega}_{0}(M)$. Then we have

$$
\mathcal{A}_{K}\left(\left[z^{+}, w^{+}\right]\right)-\mathcal{A}_{H}\left(\left[z^{-}, w^{-}\right]\right) \leq \int_{0}^{1}-\min _{x \in M}\left(K_{t}-H_{t}\right) d t .
$$

In particular, we have

$$
\lambda_{K}\left(h_{(\mathcal{H}, j)}(\beta)\right) \leq \lambda_{H}(\beta)+\int_{0}^{1}-\min _{x \in M}\left(K_{t}-H_{t}\right) d t
$$

where $h_{(\mathcal{H}, j)}(\beta)$ is the image chain of $\beta$ under the chain map $h_{(\mathcal{H}, j)}$. 


\section{Remark 2.10.}

(1) Geometrically, what this corollary proves is that under the Floer chain map, the level of Floer chains cannot increase more than the change of the corresponding Hamiltonians, more precisely, not more than $E^{-}(K-H)$. On the other hand, there is no such lower bound for the amount of the decrease of the level in general. Having a lower bound is a manifestation of some nontrivial homological property of Floer cycles.

(2) We would like to remark that a similar calculation proves there is also a uniform upper bound $C(\mathcal{H}, j)$ for the chain map over general homotopies $(\mathcal{H}, j)$ or for the Floer chain homotopy maps. Existence of such an upper estimate is crucial for the construction of these maps. This upper estimate depends on the choice of homotopy $(\mathcal{H}, j)$ and is related to the curvature estimates of the relevant Hamiltonian fibration (see [Po1], [En1]). Whether the upper bound $C(\mathcal{H}, j)$ is the same as the upper bound (2.17) for the linear homotopy $\mathcal{H}^{\text {lin }}$ is closely tied to the minimality of the Hamiltonian path $\mathcal{H}$ with respect to the Hofer metric on $\operatorname{Ham}(M, \omega)$. (See [Oh7].)

Now we recall that $C F_{*}(H)$ is not only a $\mathbb{Q}$-module but also a $\Lambda$-module where $\Lambda=\Lambda_{\omega}$ is the Novikov ring of $(M, \omega)$ : each $A \in \Gamma$ acts on $\operatorname{Crit}_{H}$ and so on $C F_{*}(H)$ by "gluing a sphere"

$$
A:[z, w] \rightarrow[z, w \# A] .
$$

Then $\partial$ is $\Lambda$-linear and induces the standard Floer homology $H F_{*}(H ; \Lambda)$ with coefficients in $\Lambda$. (See $[\mathrm{HoS}]$ for a detailed explanation of the Novikov ring $\Lambda_{\omega}$ and its action on $C F_{*}(H)$.) However the action does not preserve the filtration we defined above. Whenever we talk about filtration, we will always presume that the relevant coefficient ring is $\mathbb{Q}$. For a given nondegenerate $H$ and $\lambda \in \mathbb{R} \backslash \operatorname{Spec}(H)$, we define the relative chain group

$$
C F_{k}^{\lambda}(H):=\left\{\beta \in C F_{k}(H) \mid \lambda_{H}(\beta)<\lambda\right\} .
$$

There is one more important aspect of the filtered Floer homology; the behavior under symplectic conjugation

$$
\phi \mapsto \eta \phi \eta^{-1} ; \quad \operatorname{Ham}(M, \omega) \rightarrow \operatorname{Ham}(M, \omega)
$$

for any symplectic diffeomorphism $\eta:(M, \omega) \rightarrow(M, \omega)$. We summarize the basic facts relevant to the filtered Floer homology here.

(1) When $H \mapsto \phi, \eta_{*} H \mapsto \eta \phi \eta^{-1}$.

(2) If $H$ is nondegenerate, $\eta_{*} H$ is also nondegenerate.

(3) If $(H, J)$ is regular in the Floer theoretic sense, then so is $\left(\eta_{*} J, \eta_{*} H\right)$.

(4) There exists a natural bijection $\eta_{*}: \Omega_{0}(M) \rightarrow \Omega_{0}(M)$ defined by

$$
\eta_{*}([z, w])=([\eta \circ z, \eta \circ w])
$$

under which we have the identity

$$
\mathcal{A}_{H}([z, w])=\mathcal{A}_{\eta_{*} H}\left(\eta_{*}[z, w]\right) .
$$


(5) The $L^{2}$-gradients of the corresponding action functionals satisfy

$$
\eta_{*}\left(\operatorname{grad}_{J} \mathcal{A}_{H}\right)([z, w])=\operatorname{grad}_{\eta_{*} J}\left(\mathcal{A}_{\eta_{*} H}\right)\left(\eta_{*}([z, w])\right) .
$$

(6) If $u: \mathbb{R} \times S^{1} \rightarrow M$ is a solution of perturbed Cauchy-Riemann equation for $(H, J)$, then $\eta_{*} u=\eta \circ u$ is a solution for the pair $\left(\eta_{*} J, \eta_{*} H\right)$. In addition, all the Fredholm properties of $(J, H, u)$ and $\left(\eta_{*} J, \eta_{*} H, \eta_{*} u\right)$ are the same.

These facts imply that conjugation by $\eta$ induces the canonical filtration-preserving chain isomorphism

$$
\eta_{*}:\left(C F_{*}^{\lambda}(H), \partial_{(H, J)}\right) \rightarrow\left(C F_{*}^{\lambda}\left(\eta_{*} H\right), \partial_{\left(\eta_{*} H, \eta_{*} J\right)}\right)
$$

for any $\lambda \in \mathbb{R} \backslash \operatorname{Spec}(H)=\mathbb{R} \backslash \operatorname{Spec}\left(\eta_{*} H\right)$. In particular it induces a filtration preserving isomorphism

$$
\eta_{*}: H F_{*}^{\lambda}(H, J) \rightarrow H F_{*}^{\lambda}\left(\eta_{*} H, \eta_{*} J\right)
$$

in homology.

We close this section by fixing our grading convention for $H F_{*}(H)$. Following the convention used in [Oh8], we will always grade the complex $C F_{*}(H)$ by the Conley-Zehnder index, denoted by $\mu_{H}([z, w])$ of its generators $[z, w]$.

To make a comparison of this grading with the gradings of quantum or Morse cohomology, we recall that solutions of the negative gradient flow equation of $-f$, i.e., of the positive gradient flow of $f$

$$
\dot{\chi}-\operatorname{grad} f(\chi)=0
$$

correspond to the negative gradient flow of the action functional $\mathcal{A}_{\epsilon f}$. This gives rise to the relation between the Morse indices $\mu_{-\epsilon f}^{\text {Morse }}(p)$ and the Conley-Zehnder indices $\mu_{\epsilon f}([p, \widehat{p}])$ where $\widehat{p}: D^{2} \rightarrow M$ is the constant disc $\widehat{p}(z) \equiv p$ :

$$
\mu_{\epsilon f}([p, \widehat{p}])=\mu_{-\epsilon f}^{M o r s e}(p)-n
$$

in our convention [Oh8]. On the other hand, obviously we have

$$
\mu_{-\epsilon f}^{\text {Morse }}(p)-n=\left(2 n-\mu_{\epsilon f}^{\text {Morse }}(p)\right)-n=n-\mu_{\epsilon f}^{\text {Morse }}(p) .
$$

Our grading convention of $C F_{k}(\epsilon f)$ is

$$
k=\mu_{\epsilon f}([p, \widehat{p}])
$$

which related to the Morse index of $p \in$ Crit $\epsilon f$ by (2.20). Now we relate this grading convention to the usual grading convention of the quantum cohomology ring $Q H^{*}(M)$.

This grading convention is translated to the standard grading of the quantum cohomology via the duality map

$$
\text { b }: Q H^{n-k}(M) \rightarrow Q H_{n+k}(M)
$$


Under this grading convention, we have the following grading preserving isomorphisms

$$
Q H^{n-k}(M) \rightarrow Q H_{n+k}(M) \rightarrow H Q_{n+k}(-\epsilon f) \rightarrow H F_{k}(\epsilon f) \rightarrow H F_{k}(H) .
$$

(See [section 4.1, Oh8] for more explanations.) Recall that when a nondegenerate Hamiltonian $H$ is given, we can represent this Floer homology class by a Floer Novikov cycle $\alpha \in C F_{*}(H)$. We denote the corresponding Floer homology class by $a_{H}^{b}$ and mostly suppressing $H$ from the notation as long as the meaning is clear.

Here we recall that $H Q_{*}(-\epsilon f)$ is the Morse homology group associated to the complex

$$
\left(C M_{*}(-\epsilon f), \partial^{\text {Morse }}\right) \otimes \Lambda
$$

a Morse theoretic realization of the quantum chain complex and coincides with the Floer complex $\left(C F_{*-k}(\epsilon f), \partial\right)$. This fact is one of the important ingredients in the mini-max theory via the chain level Floer theory. We refer to [Oh5,7-9] for the detailed illustration of previous usages of this important fact, first observed by Floer himself [Fl3].

We have also shown in [Oh8] that with this grading convention the pants product, denoted by $*$, has degree $-n$ :

$$
*: H F_{k}(H) \otimes H F_{\ell}(K) \rightarrow H F_{(k+\ell)-n}(H \# K) .
$$

This suggests that it is also natural to consider the shift functor $[n]$ and the shifted complex $C F[n](H)$ by

$$
C F[n]_{k}(H):=C F_{k+n}(H), \quad \partial_{[n]}:=(-1)^{n} \partial
$$

and the corresponding homology $H F[n](H)$. In this shifted complex, the pants product $*$ has degree zero, i.e., it satisfies

$$
*: H F[n]_{k}(H) \otimes H F[n]_{\ell}(K) \rightarrow H F[n]_{(k+\ell)}(H \# K) .
$$

Finally we mention that our grading convention is consistent with the quantum product

$$
\cdot: Q H^{a}(M) \otimes Q H^{b}(M) \rightarrow Q H^{a+b}(M)
$$

having degree 0 , under the graded isomorphism (2.21).

\subsection{Spectral invariants and Floer fundamental cycles}

In this section, we briefly recall basic properties of the spectral invariants constructed in $[\mathrm{Oh} 5,9]$.

First let a nondegenerate $H$ be given and choose a one-periodic $J$ such that the pair $(H, J)$ is regular in the standard Floer theoretic sense. We will call any such pair Floer regular. For each given pair $(H, J)$ and $0 \neq a \in Q H^{*}(M)$, we define the following mini-max value of the action functional $\mathcal{A}_{H}$

$$
\rho((H, J) ; a)=\inf _{\alpha}\left\{\lambda_{H}(\alpha) \mid \alpha \in \operatorname{ker} \partial_{(H, J)} \subset C F_{*}(H) \text { with }[\alpha]=a^{b}\right\}
$$

Finiteness of $\rho((H, J) ; a)$, i.e., $\rho((H, J) ; a)>-\infty$ is proven in [Oh8] and the numbers satisfy

$$
\rho((H, J) ; a)=\rho\left(\left(H, J^{\prime}\right) ; a\right)
$$

for two different choices of generic $J, J^{\prime}$, which is a consequence of (2.17). We denote the common value by $\rho(H ; a)$. Then it is proved in [Oh8] that $\rho$ satisfies the following list of axioms. 
Theorem 2.11 [Theorem I, Oh8]. Let $(M, \omega)$ be arbitrary closed symplectic manifold. For any given quantum cohomology class $0 \neq a \in Q H^{*}(M)$, we have a continuous function denoted by

$$
\rho=\rho(H ; a): C_{m}^{\infty}([0,1] \times M) \times Q H^{*}(M) \rightarrow \mathbb{R}
$$

such that they satisfy the following axioms: Let $H, F \in C_{m}^{\infty}([0,1] \times M)$ be smooth Hamiltonian functions and $a \neq 0 \in Q H^{*}(M)$. Then $\rho$ satisfies the following axioms:

(1) (Projective invariance) $\rho(H ; \lambda a)=\rho(H ; a)$ for any $0 \neq \lambda \in \mathbb{Q}$.

(2) (Normalization) For $a=\sum_{A \in \Gamma} a_{A} q^{-A}$, we have $\rho(\underline{0} ; a)=v(a)$ where $\underline{0}$ is the zero function and

$$
v(a):=\min \left\{\omega(-A) \mid a_{A} \neq 0\right\}=-\max \left\{\omega(A) \mid a_{A} \neq 0\right\} .
$$

is the (upward) valuation of $a$.

(3) (Symplectic invariance) $\rho\left(\eta_{*} H ; a\right)=\rho(H ; a)$ for any symplectic diffeomorphism $\eta$

(4) (Triangle inequality) $\rho(H \# F ; a \cdot b) \leq \rho(H ; a)+\rho(F ; b)$

(5) (C $C^{0}$-continuity) $|\rho(H ; a)-\rho(F ; a)| \leq\|H \# \bar{F}\|=\|H-F\|$ where $\|\cdot\|$ is the Hofer's pseudo-norm on $C_{m}^{\infty}([0,1] \times M)$. In particular, the function $\rho_{a}: H \mapsto \rho(H ; a)$ is $C^{0}$-continuous.

It is important to note that $\rho_{a}$ is defined for each function $H$, whether it is nondegenerate or not, and continuously extends to $C^{0}$ functions. Recall that the Lie algebra of $\operatorname{Ham}(M, \omega)$ as a vector space is 'universal'; that is, independent of the symplectic form $\omega$. This enables one to study the change of invariants $\rho(H ; a)$ under the change of symplectic form for each fixed $H$. This will be a subject of future study.

Here the symplectic invariance (3) follows from the discussion on symplectic conjugation in subsection 2.2, which in particular implies that $\eta_{*}$ induces a canonical filtration-preserving bijection between the Floer cycles of $(H, J)$ and $\left(\eta_{*} H, \eta_{*} J\right)$. This in particular implies that the mini-max values $\rho(H ; a)$ and $\rho\left(\eta_{*} H ; a\right)$ are the same.

We next state the following theorem which will be used later in the proof of the identity (5.5) for the class $a=1$.

Theorem 2.12 (Homotopy Invariance). Assume that the spectrality axiom (1.16) holds for nondegenerate Hamiltonians. Let $F$ and $K$ be arbitrary Hamiltonians satisfying $F \sim K$. Then we have

$$
\rho(F ; a)=\rho(K ; a) .
$$

In particular, the function $F \mapsto \rho(F ; a)$ pushes down to a continuous function $\rho_{a}: \widetilde{\operatorname{Ham}}(M, \omega) \rightarrow \mathbb{R}$ in the natural topology on $\widetilde{\operatorname{Ham}}(M, \omega)$.

In [Oh8], we gave a proof of this homotopy invariance based on the spectrality axiom for the rational $(M, \omega)$. See the recent preprint (math.SG/0406449) of the author for the proof of spectrality axiom for the irrational case.

To understand the value given by (2.23) in practice, it is crucial to find a good Floer fundamental cycle $\alpha \in C F_{n}(H)$ for a given regular $(H, J)$. An important 
task is to construct such a cycle. One good way of constructing such a cycle is to transfer a corresponding Morse cycle via the Floer chain map, which has been frequently used by the author [Oh7-9]. We explain this procedure now. First, we fix a generic Morse function $f$ and represent the fundamental cycle $1^{b}=[M]$ by a Morse cycle of $\left(-\epsilon f, g_{J_{0}}\right)$

$$
\alpha=\sum_{j} a_{j}\left[x_{j}, \widehat{x}_{j}\right], \quad a_{j} \in \mathbb{Q}
$$

with $x_{j} \in \mathrm{Crit}_{2 n}(-\epsilon f)$ and $\widehat{x}_{j}$ is the constant bounding disc. This particular fundamental cycle has the important property that the cycle cannot be pushed down in the sense of [Oh4]. We recall the following definition from [Oh7] which will be useful for our later discussions.

Definition 2.13. Let $(H, J)$ be a Floer regular pair so that the Floer complex $\left(C F_{*}(H), \partial_{(H, J)}\right)$ is well-defined. Let $\alpha$ be a Floer cycle of $H$ and $a \in Q H^{*}(M)$ be the corresponding quantum cohomology class with $[\alpha]=a^{b}$. We call the Floer cycle $\alpha$ tight if it realizes the mini-max value, i.e.,

$$
\lambda_{H}(\alpha)=\rho(H ; a)
$$

and we call the corresponding critical value a homologically essential critical value of $\mathcal{A}_{H}$.

Using the fact that, when $\epsilon>0$ is sufficiently small, this Morse cycle also provides a Floer cycle of $\epsilon f$ that represents the quantum cohomology class 1, we transfer $\alpha$ to

$$
\alpha_{H}=h_{(\mathcal{H}, j)}(\alpha)
$$

by the chain map $h_{(\mathcal{H}, j)}: C F_{n}(\epsilon f) \rightarrow C F_{n}(H)$ along a homotopy $(\mathcal{H}, j)$ to the cycle $h_{(\mathcal{H}, j)}\left(\alpha_{H}\right)$. This will then represent a Floer fundamental cycle for $H$ by the invariance of the Floer homology under the homotopy of Hamiltonians.

In this paper and others [Oh5,7-9], we have often used the linear homotopy $\mathcal{H}$ defined by

$$
\mathcal{H}: s \mapsto(1-s) \epsilon f+s H
$$

to represent a Floer fundamental cycle of the given Hamiltonian $H$ in this way. Following [Oh7], we call this particular fundamental cycle a canonical Floer fundamental cycle. We emphasize that this cycle, however, depends on the initial Morse function $f$ (and also on the choice of the homotopy $(\mathcal{H}, j)$ ). In general the transferred cycles will not be tight. Understanding under what condition of $H$ the transferred cycle remains tight provides much information on the length minimizing property of Hofer's geodesics. (See [Oh7] for further discussions on this issue.)

\subsection{Comparison of two Cauchy-Riemann equations}

In this section, we explain the relation between Floer's standard perturbed Cauchy-Riemann equation for $u: \mathbb{R} \times S^{1} \rightarrow M$

$$
\left\{\begin{array}{l}
\frac{\partial u}{\partial \tau}+J_{t}\left(\frac{\partial u}{\partial t}-X_{H}(u)\right)=0 \\
\int\left|\frac{\partial v}{\partial \tau}\right|_{J_{t}}^{2}<\infty
\end{array}\right.
$$


and its mapping cylinder version $v: \mathbb{R} \times \mathbb{R} \rightarrow M$

$$
\left\{\begin{array}{l}
\frac{\partial v}{\partial \tau}+J_{t}^{\prime} \frac{\partial v}{\partial t}=0 \\
\phi(v(\tau, t+1))=v(\tau, t), \quad \int\left|\frac{\partial v}{\partial \tau}\right|_{J_{t}^{\prime}}^{2}<\infty
\end{array}\right.
$$

where $\phi=\phi_{1}^{H}$. We often restrict $v$ to $\mathbb{R} \times[0,1]$ and consider it as a map from $\mathbb{R} \times[0,1]$ that satisfies $\phi(v(\tau, 1))=v(\tau, 0)$. The present author previously exploited a similar correspondence in $[\mathrm{Oh} 3,4]$ in the 'open string' context of Lagrangian submanifolds for the same purpose, and call the former version of Floer homology the dynamical and the latter geometric. We do the same here.

Before explaining the correspondence, we would like to point out one subtle difference between the case of the 'open string' version [Oh3,4] and the current 'closed string' version. In the point of view of transforming the equations (2.27), (2.28) from one to the other, we must consider a periodic family $\left\{J_{t}\right\}_{0 \leq t \leq 1}$ for (2.27), which was not necessary for the open string version. Then for any given solution $u=u(\tau, t): \mathbb{R} \times S^{1} \rightarrow M$, we 'open up' $u$ along $t=0 \equiv 1$ and define the map

$$
v: \mathbb{R} \times[0,1] \rightarrow M
$$

by

$$
v(\tau, t)=\left(\phi_{H}^{t}\right)^{-1}(u(\tau, t))
$$

and then extend to $\mathbb{R}$ so that $\phi(v(\tau, t+1))=v(\tau, t)$. A simple computation shows that the map satisfies (2.28), provided the family $J^{\prime}=\left\{J_{t}^{\prime}\right\}_{0 \leq t \leq 1}$ is defined by

$$
J_{t}^{\prime}=\left(\phi_{H}^{t}\right)^{*} J_{t}
$$

for the given periodic family $J_{t}$ used for the equation (2.27). By definition, this family $J^{\prime}$ of almost complex structure satisfies

$$
J^{\prime}(t+1)=\phi^{*} J^{\prime}(t)
$$

The condition

$$
\phi(v(\tau, t+1))=v(\tau, t)
$$

enables us to consider the map

$$
v: \mathbb{R} \times \mathbb{R} \rightarrow M
$$

as a pseudo-holomorphic section of the 'mapping cylinder'

$$
E_{\phi}:=\mathbb{R} \times M_{\phi}=\mathbb{R} \times \mathbb{R} \times M /(\tau, t+1, \phi(x)) \sim(\tau, t, x)
$$

where $M_{\phi}$ is the mapping circle defined by

$$
M_{\phi}:=\mathbb{R} \times M /(t+1, \phi(x)) \sim(t, x) .
$$

Note that the product symplectic form $d \tau \wedge d t+\omega$ on $\mathbb{R} \times \mathbb{R} \times M$ naturally projects to $E_{\phi}$ since $\phi$ is symplectic, and so $E_{\phi}$ has the structure of a Hamiltonian fibration. 
In this setting, $v: \mathbb{R} \times \mathbb{R} \rightarrow M$ can be regarded as the section $s: \mathbb{R} \times S^{1} \rightarrow E_{\phi}$ defined by

$$
s(\tau, t)=[\tau, t, v(\tau, t)]
$$

which becomes a pseudo-holomorphic section of $E_{\phi}$ for a suitably defined almost complex structure. (See [section 3, Oh9] for the precise formulations.)

One advantage of the mapping cylinder version over the more standard dynamical version (2.27) is that its dependence on the Hamiltonian $H$ is much weaker than in the latter. Indeed, this mapping cylinder version can be put into the general framework of Hamiltonian fibrations with given fixed monodromy of the fibration at infinity as in [En1]. This framework turns out to be essential to prove the triangle inequality of the spectral invariants. (See section 5).

Another important ingredient is the formula for the energy: For the given periodic family $J=\left\{J_{t}\right\}_{0 \leq t \leq 1}$, we define the energy of the map $u: \mathbb{R} \times S^{1} \rightarrow M$ by

$$
E_{J}(u)=\frac{1}{2} \int_{\mathbb{R} \times S^{1}}\left(\left|\frac{\partial u}{\partial \tau}\right|_{J_{t}}^{2}+\left|\frac{\partial u}{\partial t}-X_{H}(u)\right|_{J_{t}}^{2}\right) d t d \tau
$$

for (2.27). Similarly for the given $J^{\prime}=\left\{J_{t}^{\prime}\right\}_{0 \leq t \leq 1} \in j_{\left(J_{0}, \phi\right)}$, we define the energy of the map $v: \mathbb{R} \times[0,1] \rightarrow M$ by

$$
E_{J^{\prime}}(v)=\frac{1}{2} \int_{\mathbb{R} \times[0,1]}\left(\left|\frac{\partial v}{\partial \tau}\right|_{J_{t}^{\prime}}^{2}+\left|\frac{\partial v}{\partial t}\right|_{J_{t}^{\prime}}^{2}\right) d t d \tau
$$

This energy is the vertical part of the energy of the section $s: \mathbb{R} \times S^{1} \rightarrow E_{\phi}$ defined above with respect to a suitably chosen almost complex structure $\widetilde{J}$ on $E_{\phi}$. (See [section 3, Oh9] for more explanation.) Note that because of (2.29)-(2.30), one can replace the domain of integration $\mathbb{R} \times[0,1]$ by any fundamental domain of the covering projection

$$
\mathbb{R} \times \mathbb{R} \rightarrow \mathbb{R} \times(\mathbb{R} / \mathbb{Z})
$$

without changing the integral. The choice of $\mathbb{R} \times[0,1]$ is one such choice.

The following identity plays an important role in the proof of the nondegeneracy of the invariant norm we construct later. The proof is a straightforward computation and left to the readers.

Lemma 2.14. Let $J=\left\{J_{t}\right\}_{0 \leq t \leq 1}$ be a periodic family and define $J^{\prime}=\left\{J_{t}^{\prime}\right\}_{0 \leq t \leq 1}$ by

$$
J_{t}^{\prime}=\left(\phi_{H}^{t}\right)^{*} J_{t} .
$$

Let $u: \mathbb{R} \times S^{1} \rightarrow M$ be any smooth map and $v: \mathbb{R} \times[0,1] \rightarrow M$ be the map defined by

$$
v(\tau, t)=\left(\phi_{H}^{t}\right)^{-1}(u(\tau, t)) .
$$

Then we have

$$
E_{J}(u)=E_{J^{\prime}}(v) .
$$




\section{§3. Definition of the spectral norm}

In this section, we prove Theorem II in the introduction. We first recall

$$
\gamma(H)=\rho(H ; 1)+\rho(\bar{H} ; 1) \geq 0 .
$$

We now relate $\rho(H ; 1)$ with the negative Hofer length $E^{-}(H)$.

Theorem 3.1. For any $H$ and $0 \neq a \in Q H^{*}(M)$, we have

$$
-E^{+}(H)+v(a) \leq \rho(H ; a) \leq E^{-}(H)+v(a) .
$$

In particular for any classical cohomology class $b \in H^{*}(M) \hookrightarrow Q H^{*}(M)$, we have

$$
-E^{+}(H) \leq \rho(H ; b) \leq E^{-}(H)
$$

for any Hamiltonian $H$.

Proof. We first recall the following general inequality

$$
\int_{0}^{1}-\max (H-K) d t \leq \rho(H ; a)-\rho(K ; a) \leq \int_{0}^{1}-\min (H-K) d t .
$$

proven in [Oh 9], which can be rewritten as

$$
\rho(K ; a)+\int_{0}^{1}-\max (H-K) d t \leq \rho(H ; a) \leq \rho(K ; a)+\int_{0}^{1}-\min (H-K) d t .
$$

Now let $K \rightarrow 0$ which results in

$$
\rho(0 ; a)+\int_{0}^{1}-\max (H) d t \leq \rho(H ; a) \leq \rho(0 ; a)+\int_{0}^{1}-\min (H) d t .
$$

By the normalization axiom from subsection 2.3, we have $\rho(0 ; a)=v(a)$ which turns (3.5) to

$$
v(a)-E^{+}(H) \leq \rho(H ; a) \leq v(a)+E^{-}(H)
$$

for any $H$. (3.3) immediately follow from the definitions and the identity $v(b)=0$ for a classical cohomology class $b$. This finishes the proof.

Applying (3.3) to $b=1$, we derive $\rho(H ; 1) \leq E^{-}(H)$ and $\rho(\bar{H} ; 1) \leq E^{-}(\bar{H})$ for arbitrary $H$, and hence

$$
\gamma(H) \leq\|H\| .
$$

The nonnegativity (3.1) leads us to the following definition.

Definition 3.2. We define $\gamma: \operatorname{Ham}(M, \omega) \rightarrow \mathbb{R}_{+}$by

$$
\gamma(\phi):=\inf _{H \mapsto \phi}(\rho(H ; 1)+\rho(\bar{H} ; 1))
$$

and call this the spectral (pseudo)-norm.

The following theorem shows that $\gamma$ is indeed a norm on $\operatorname{Ham}(M, \omega)$ that is invariant under the conjugate action of symplectic diffeomorphisms which in turn implies the biinvariance of the norm. 
Theorem 3.3. Let $\gamma$ be as above. Then $\gamma: \operatorname{Ham}(M, \omega) \rightarrow \mathbb{R}_{+}$enjoys the following properties.

(1) $\phi=i d$ if and only if $\gamma(\phi)=0$

(2) $\gamma\left(\eta^{-1} \phi \eta\right)=\gamma(\phi)$ for any symplectic diffeomorphism $\eta$

(3) $\gamma(\phi \psi) \leq \gamma(\phi)+\gamma(\psi)$

(4) $\gamma\left(\phi^{-1}\right)=\gamma(\phi)$

(5) $\gamma(\phi) \leq\|\phi\|$

In the remaining section, we will give the proofs of these statements postponing the most non-trivial statement, nondegeneracy, to section 5. We split our proof of Theorem 3.3 item by item.

Proof of (2). We recall the symplectic invariance of spectral invariants $\rho(H ; a)=$ $\rho\left(\eta_{*} H ; a\right)$. Applying this to $a=1$, we derive the identity

$$
\begin{aligned}
\gamma(\phi) & =\inf _{H \mapsto \phi}(\rho(H ; 1)+\rho(\bar{H} ; 1)) \\
& =\inf _{H \mapsto \phi}\left(\rho\left(\eta_{*} H ; 1\right)+\rho\left(\overline{\eta_{*} H} ; 1\right)\right)=\gamma\left(\eta^{-1} \phi \eta\right),
\end{aligned}
$$

which finishes the proof.

Proof of (3). We first recall the triangle inequality

$$
\rho(H \# K ; 1) \leq \rho(H ; 1)+\rho(K ; 1)
$$

and

$$
\rho(\bar{K} \# \bar{H} ; 1) \leq \rho(\bar{K} ; 1)+\rho(\bar{H} ; 1) .
$$

Adding up (3.7) and (3.8), we have

$$
\begin{aligned}
(\rho(H \# K ; 1) & +\rho(\bar{H} \# \bar{K} ; 1)) \\
& \leq(\rho(H ; 1)+\rho(\bar{H} ; 1))+(\rho(K ; 1)+\rho(\bar{K} ; 1)) .
\end{aligned}
$$

Now let $H \mapsto \phi$ and $K \mapsto \psi$. Because $H \# K$ generates $\phi \psi$ and $\bar{K} \# \bar{H}$ generates $\psi^{-1} \phi^{-1}=(\phi \psi)^{-1}$, we have

$$
\gamma(\phi \psi) \leq \rho(H \# K ; 1)+\rho(\bar{H} \# \bar{K} ; 1)
$$

and hence

$$
\gamma(\phi \psi) \leq(\rho(H ; 1)+\rho(\bar{H} ; 1))+(\rho(K ; 1)+\rho(\bar{K} ; 1))
$$

from (3.9). By taking the infimum of the right hand side over all $H \mapsto \phi$ and $K \mapsto \psi,(3)$ is proved.

Proof of (4). The proof immediately follows from the observation that the definition of $\gamma$ is symmetric over the map $\phi \mapsto \phi^{-1}$.

Proof of (5). This is an immediate consequence of (3.6) with its infimum taken over $H \mapsto \phi$.

It now remains to prove nondegeneracy of $\gamma$, which we will do in section 5 modulo the proof of an existence theorem, Theorem 5.4. Proof of the latter will be given in section 8 . 


\section{The $\epsilon$-regularity theorem and its consequences}

As in the proof of nondegeneracy of Hofer's norm in [Ho] and [LM], we will prove nondegeneracy of $\gamma$ by comparing it with a geometric invariant of $\phi$ independent of its generating Hamiltonians. In $[\mathrm{Ho}]$ and $[\mathrm{LM}]$, the authors use the maximal Gromov area $\lambda>0$ of symplectic balls $B(\lambda)$ and then prove the lower bound of $\|H\|$ for $H$ s which satisfy $\phi_{H}^{1}(B(\lambda)) \cap B(\lambda)=\emptyset$. Here we denote by $B(\lambda)$ the image of any symplectic embedding $g: B^{2 n}(r) \subset \mathbb{C}^{n} \rightarrow(M, \omega)$, and call the image $g(0)$ the center of the symplectic embedding $g$ or $B(\lambda)$. It seems to be useful to formalize this invariant of general Hamiltonian diffeomorphisms in the following way.

Definition 4.1. Let $\phi$ be an arbitrary Hamiltonian diffeomorphism. We define

$$
\text { bArea }(\phi):=\sup _{B}\left\{\lambda=\pi r^{2} \mid \phi(B(\lambda)) \cap B(\lambda)=\emptyset\right\}
$$

and call it the ball-area of $\phi$.

For the lower estimate of $\gamma(\phi)$, we will use another geometric invariant of $\phi$, instead of the ball area, which much better suits our chain level Floer theory. The rest of this section is occupied by the description of this invariant and the proof of its nontriviality.

Suppose $\phi$ is a nondegenerate Hamiltonian diffeomorphism. Denote by $J_{0}$ a compatible almost complex structure on $(M, \omega)$. For given such a pair $\left(\phi, J_{0}\right)$, we consider paths $J^{\prime}:[0,1] \rightarrow \mathcal{J}_{\omega}$ with

$$
J^{\prime}(0)=J_{0}, \quad J^{\prime}(1)=\phi^{*} J_{0}
$$

and define $j_{\left(\phi, J_{0}\right)}$ to be the set of such paths. We extend $J^{\prime}$ to $\mathbb{R}$ so that

$$
J^{\prime}(t+1)=\phi^{*} J^{\prime}(t)
$$

For each given $J^{\prime} \in j_{\left(\phi, J_{0}\right)}$, we define the constant

$$
\begin{aligned}
A_{S}\left(\phi, J_{0} ; J^{\prime}\right)=\inf \left\{\omega([u]) \mid u: S^{2} \rightarrow M\right. \text { non-constant and } \\
\text { satisfying } \left.\bar{\partial}_{J_{t}^{\prime}} u=0 \text { for some } t \in[0,1]\right\} .
\end{aligned}
$$

A priori it is not obvious whether $A_{S}\left(\phi, J_{0} ; J^{\prime}\right)$ is not zero. We will prove this using the so called $\epsilon$-regularity theorem. This kind of $\epsilon$-regularity theorem is one of the fundamental ingredients, first introduced by Sachs and Uhlenbeck [SU], for the study of compactness and regularity theory in the geometric analysis of harmonic maps, minimal surfaces and others including the Gromov compactness theorem of pseudo-holomorphic maps. We state a parametric version of the $\epsilon$-regularity theorem stated in [Oh1] in the context of pseudo-holomorphic curves.

Let $g$ be any almost Kähler metric of $(M, \omega)$, i.e., $g=\omega\left(\cdot, J_{\text {ref }} \cdot\right)$ for some fixed almost complex structure $J_{\text {ref }}$ compatible to $\omega$. Denote by $|\cdot|$ the norm associated to this fixed metric. We will always assume that such an almost Kähler metric $g$ is given and all the norms used are in terms of this fixed metric, unless otherwise said. We denote the derivative of $u$ by $D u$. 
Lemma 4.2 ( $\epsilon$-Regularity Theorem) [Proposition 3.3, Oh1]. We denote by $D=D^{2}(1)$ the unit open disc . Let $J_{0}$ be any almost complex structure and let $u: D \rightarrow M$ be a $J_{0}$-holomorphic map. Then there exists some $\epsilon=\epsilon\left(g, J_{0}\right)>0$ such that if $\int_{D}|D u|^{2}<\epsilon$, then for any smaller disc $D^{\prime}=D^{2}(r)$ with $\bar{D}^{\prime} \subset D$, we have

$$
\|D u\|_{\infty, D^{\prime}}:=\max _{z \in D^{\prime}}|D u(z)| \leq C
$$

where $C>0$ depends only on $g, \epsilon, J_{0}$ and $D^{\prime}$, not on $u$. Furthermore, the same $C^{1}$-bound holds for any compact family $K$ of compatible almost complex structures this time with $\epsilon=\epsilon(g, K)$ and $C=C\left(g, \epsilon, K, D^{\prime}\right)$ depending on $K$.

An immediate corollary of this $\epsilon$-regularity theorem is the following uniform $C^{1}$-estimate of pseudo-holomorphic curves.

Corollary 4.3. Let $J^{\prime} \in j_{\left(\phi, J_{0}\right)}$. Then there exists an $\epsilon=\epsilon\left(J^{\prime}\right)>0$ such that if $\omega(u)<\epsilon$, then we have

$$
\|D u\|_{\infty}:=\max _{z \in S^{2}}|D u(z)| \leq C
$$

for any $J_{t}^{\prime}$-holomorphic sphere $u: S^{2} \rightarrow M$ and for any $t \in[0,1]$ where $C=C\left(\epsilon, J^{\prime}\right)$ does not depend on $u$.

Proof. Let $u$ be $J_{t_{0}}$-holomorphic for $t_{0} \in[0,1]$. We choose an open covering of $S^{2}$ by a finite number of discs $\left\{D_{\alpha}\right\}$ with a fixed size. Obviously we have

$$
\frac{1}{2} \int_{D_{\alpha}}|D u|^{2} \leq \frac{1}{2} \int_{S^{2}}|D u|^{2} .
$$

On the other hand, since $M$ and $[0,1]$ are compact, there exists a uniform constant $K>0$ such that

$$
\frac{1}{\sqrt{K}}|\cdot| \leq|\cdot|_{J_{t}^{\prime}} \leq \sqrt{K}|\cdot|
$$

for all $t \in[0,1]$, where we recall $|\cdot|$ is the norm measured in terms of the metric $g$ associated to the fixed $J_{\text {ref }}$. Therefore combining (4.4) and (4.5), we derive

$$
\frac{1}{2} \int_{D_{\alpha}}|D u|^{2} \leq \frac{K}{2} \int_{S^{2}}|D u|_{J_{t_{0}}}^{2}=K \omega(u) .
$$

Here we used the well-known identity

$$
\frac{1}{2} \int_{S^{2}}|D u|_{J_{0}}^{2}=\omega(u)
$$

for $J_{0}$-holomorphic map $u: S^{2} \rightarrow M$ where $J_{0}$ is compatible to $\omega$. We emphasize that for this identity to hold the norm $|\cdot|_{J_{0}}$ and the pseudo-holomorphic map $u$ must be associated to the same compatible almost complex structure $J_{0}$. This is the reason why we need to use (4.5) to relate the harmonic energy $\frac{1}{2} \int_{S^{2}}|D u|^{2}$ of $J_{t_{0}}$-holomorphic map $u$, which is measured by the reference metric $g=\omega\left(\cdot, J_{r e f} \cdot\right)$, to the symplectic area $\omega(u)$.

Since $\int_{D_{\alpha}}|D u|^{2}$ is invariant under the conformal change of metric of the domain, we may assume the radius of $D_{\alpha}$ is one for all $\alpha$. Choose $\epsilon>0$ so that the $C^{1}$-bound in Lemma 4.2 holds for $K \epsilon$. Then we choose a smaller discs $D_{\alpha}^{\prime}$ so that $\bar{D}_{\alpha}^{\prime} \subset D_{\alpha}$ and $\left\{D_{\alpha}^{\prime}\right\}$ still covers $S^{2}$ for each $\alpha$. Then we apply Lemma 4.2 for each $\alpha$ and obtain (4.3).

With Corollary 4.3 at our disposal, we prove the following strict positivity result. We note that we cannot rule out the possibility $A_{S}\left(\phi, J_{0} ; J^{\prime}\right)=\infty$ as in the weakly exact case where $A_{S}\left(\phi, J_{0} ; J^{\prime}\right)=\infty$ for all choices of $\left(J_{0} ; J^{\prime}\right)$. 
Proposition 4.4. Let $\phi, J_{0}$ and $J^{\prime}$ be as above. Then we have

$$
A_{S}\left(\phi, J_{0} ; J^{\prime}\right)>0 \text {. }
$$

Proof. Suppose $A\left(\phi, J_{0} ; J^{\prime}\right)=0$. Then there exists a sequence $t_{j} \in[0,1]$ and a sequence of non-constant maps $u_{j}: S^{2} \rightarrow M$ such that $u_{j}$ is $J_{t_{j}}$-holomorphic and

$$
\omega\left(u_{j}\right)=E_{J_{t_{j}}}\left(u_{j}\right) \rightarrow 0
$$

as $j \rightarrow \infty$. By choosing a subsequence of $t_{j}$, again denoted by $t_{j}$, we may assume that $t_{j} \rightarrow t_{\infty} \in[0,1]$ and so $J_{t_{j}}$ converges to $J_{t_{\infty}}$ in the $C^{\infty}$-topology. We choose a sufficiently large $N \in \mathbb{Z}_{+}$so that

$$
E_{J_{t_{j}}}\left(u_{j}\right)=\omega\left(u_{j}\right)<\epsilon\left(J^{\prime}\right)
$$

for all $j \geq N$, where $\epsilon\left(J^{\prime}\right)$ is the constant $\epsilon$ provided in Corollary 4.3. Then we have the uniform $C^{1}$-bound

$$
0<\left\|D u_{j}\right\|_{\infty} \leq C\left(\epsilon, J^{\prime}\right)
$$

The Ascoli-Arzela theorem then implies that there exists a subsequence, again denoted by $u_{j}$, such that $u_{j}$ converges uniformly to a continuous map $u_{\infty}: S^{2} \rightarrow M$. Recalling that all the $u_{j}$ are $J_{t_{j}}$-holomorphic and $J_{t_{j}}$ converges to $J_{t_{\infty}}$ in the $C^{\infty}$ topology, the standard boot-strap argument implies that $\left\{u_{j}\right\}$ converges to $u_{\infty}$ in the $C^{1}$ topology (and so in the $C^{\infty}$-topology). However we have

$$
E_{J_{\infty}}\left(u_{\infty}\right)=\lim _{j \rightarrow \infty} E_{J_{t_{j}}}\left(u_{j}\right)=0
$$

and hence $u_{\infty}$ must be a constant map, say $u_{\infty} \equiv x \in M$. Therefore $\left\{u_{j}\right\}$ converges to the point $x$ in the $C^{\infty}$-topology. In particular, if $j$ is sufficiently large, then the image of $u_{j}$ is contained in a (contractible) Darboux neighborhood of $x$. Therefore we must have $\omega\left(\left[u_{j}\right]\right)=0$ and in turn

$$
E_{J_{t_{j}}}\left(u_{j}\right)=0
$$

for all sufficiently large $j$, because $E_{J_{t_{j}}}(u)=\omega(u)$ holds for any $J_{t_{j}}$-holomorphic curve $u$. This contradicts the assumption that $u_{j}$ is non-constant. This finishes the proof.

Next for each given $J^{\prime} \in j_{\left(\phi, J_{0}\right)}$, we consider the equation of $v: \mathbb{R} \times \mathbb{R} \rightarrow M$

$$
\left\{\begin{array}{l}
\frac{\partial v}{\partial \tau}+J_{t}^{\prime} \frac{\partial v}{\partial t}=0 \\
\phi(v(\tau, t+1))=v(\tau, t), \quad \int_{\mathbb{R} \times[0,1]}\left|\frac{\partial v}{\partial \tau}\right|_{J_{t}^{\prime}}^{2}<\infty .
\end{array}\right.
$$


Lemma 4.5. Assume that $\phi$ is nondegenerate. For any solution $v$ of (4.7), we consider the paths

$$
v(\tau):[0,1] \rightarrow M, \quad v(\tau)(t):=v(\tau, t) .
$$

Then $v(\tau)$ uniformly converges to a fixed point $p \in$ Fix $\phi$ as $\tau \rightarrow \pm \infty$. We simply say

$$
v( \pm \infty) \in \text { Fix } \phi .
$$

Proof. We will prove this only as $\tau \rightarrow \infty$, since the case as $\tau \rightarrow-\infty$ will be the same. The finite energy condition

$$
\int_{\mathbb{R} \times[0,1]}\left|\frac{\partial v}{\partial \tau}\right|_{J_{t}^{\prime}}^{2}<\infty
$$

implies that there exists a sequence $\tau_{j} \rightarrow \infty$ such that

$$
\int_{0}^{1}\left|\frac{\partial v}{\partial \tau}\left(\tau_{j}, \cdot\right)\right|_{J_{t}^{\prime}}^{2} d t \rightarrow 0
$$

as $j \rightarrow \infty$. Together with the nondegeneracy assumption on $\phi$ and the choice of $J^{\prime}$, this implies an exponential decay of $\left|\frac{\partial v}{\partial \tau}\left(\tau_{j}, \cdot\right)\right|_{J_{t}^{\prime}}^{2}$ as $\tau \rightarrow \infty$. (See [Fl1, SZ] for such a proof.) Therefore we have

$$
\int_{0}^{1}\left|\frac{\partial v}{\partial \tau}(\tau, \cdot)\right|_{J_{t}^{\prime}}^{2} d t \rightarrow 0 \quad \text { as } \tau \rightarrow \infty
$$

Since $M$ is compact, using the compactness of the Sobolev embedding $W^{1,2}([0,1]) \hookrightarrow$ $C^{0}([0,1])$ and then the exponential decay property, we derive that $v(\tau):[0,1] \rightarrow M$ converges uniformly on $[0,1]$ to a constant point $p \in M$. Due to the boundary condition $\phi(v(\tau, t+1))=v(\tau, t)$, we have $\phi(v(\tau, 1))=v(\tau, 0)$ which immediately implies that the point $p$ must satisfy $\phi(p)=p$, i.e., $p$ is a fixed point of $\phi$. This finishes the proof.

Now it is a crucial matter to produce a non-constant solution of (4.7). For this purpose, using the fact that $\phi \neq i d$, we choose a symplectic ball $B(\lambda)$ such that

$$
\phi(B(\lambda)) \cap B(\lambda)=\emptyset
$$

where $B(\lambda)$ is the image of a symplectic embedding $g: B^{2 n}(r) \rightarrow B(\lambda) \subset M$ of the standard Euclidean ball $B^{2 n}(r) \subset \mathbb{C}^{n}$ of radius $r$ with $\lambda=\pi r^{2}$. We then study (4.7) together with

$$
v(0,0) \in B(\lambda) .
$$

Because of (4.8) and (4.9), it follows

$$
v( \pm \infty) \in \text { Fix } \phi \subset M \backslash B(\lambda) .
$$

Therefore any such solution cannot be constant.

We now define the constant

$$
A_{D}\left(\phi, J_{0} ; J^{\prime}\right):=\inf _{v}\left\{\int_{\mathbb{R} \times[0,1]} v^{*} \omega \mid v \text { non-constant solution of }(4.7)\right\}
$$

for each $J^{\prime} \in j_{\left(\phi, J_{0}\right)}$. Obviously we have $A_{D}\left(\phi, J_{0} ; J^{\prime}\right) \geq 0$.

Now we will prove $A_{D}\left(\phi, J_{0} ; J^{\prime}\right) \neq 0$. We first derive the following simple lemma. 
Lemma 4.6. Suppose that $u: \mathbb{R} \times S^{1} \rightarrow M$ is any finite energy solution of

$$
\left\{\begin{array}{l}
\frac{\partial u}{\partial \tau}+J_{t}\left(\frac{\partial u}{\partial t}-X_{H}(u)\right)=0 \\
\int\left|\frac{\partial u}{\partial \tau}\right|_{J_{t}}^{2}<\infty
\end{array}\right.
$$

that satisfies

$$
u(-\infty, t)=u(\infty, t) .
$$

Then $\int_{\mathbb{R} \times S^{1}} u^{*} \omega$ converges, and we have

$$
E_{J}(u)=\int_{\mathbb{R} \times S^{1}} u^{*} \omega
$$

Proof. First note that when $H$ is nondegenerate, any finite energy solution has well-defined asymptotic limits $z^{ \pm}=u( \pm \infty)$. Then we pick any bounding discs $w^{ \pm}$ of $z^{ \pm}$such that $w^{+} \sim w^{-} \# u$. Now (4.14) is an immediate consequence of (2.14) applied to $\mathcal{H}^{l i n} \equiv H$, since we assume (4.13), i.e., $z^{+}=z^{-}$.

With this proposition, we are ready to prove positivity of $A_{D}\left(\phi, J_{0} ; J^{\prime}\right)$. Again a priori it is possible that $A_{D}\left(\phi, J_{0} ; J^{\prime}\right)=\infty$.

Proposition 4.7. Suppose that $\phi$ is nondegenerate, and $J_{0}$ and $J^{\prime} \in j_{\left(\phi, J_{0}\right)}$ as above. Then we have

$$
A_{D}\left(\phi, J_{0} ; J^{\prime}\right)>0
$$

Proof. We prove this by contradiction. Suppose $A_{D}\left(\phi, J_{0} ; J^{\prime}\right)=0$ so that there exists a sequence of non-constant maps $v_{j}: \mathbb{R} \times[0,1] \rightarrow M$ that satisfy (4.7) and

$$
E_{J^{\prime}}\left(v_{j}\right) \rightarrow 0 \text { as } j \rightarrow \infty
$$

Therefore we have

$$
E_{J^{\prime}}\left(v_{j}\right)<\epsilon\left(J^{\prime}\right)
$$

for all sufficiently large $j^{\prime}$, where $\epsilon\left(J^{\prime}\right)$ is the constant in Lemma 4.2 and Corollary 4.3. In particular, the sequence $v_{j}$ cannot bubble off. This implies that $v_{j}$ locally uniformly converge, and in turn that $v_{j}$ must (globally) uniformly converge to a constant map because $E_{J^{\prime}}\left(v_{j}\right) \rightarrow 0$. Since there are only finitely many fixed points of $\phi$ by the nondegeneracy hypothesis, by choosing a subsequence if necessary, we conclude

$$
v_{j}(-\infty)=v_{j}(\infty)=p
$$

for all $j$ 's for some $p \in$ Fix $\phi$. Now we fix any Hamiltonian $H:[0,1] \times M \rightarrow \mathbb{R}$ that is zero near $t=0,1$ and with $H \mapsto \phi$, and consider the following maps

$$
u_{j}: \mathbb{R} \times S^{1} \rightarrow M, \quad u_{j}(\tau, t):=\left(\phi_{H}^{t}\right)\left(v_{j}(\tau, t)\right) .
$$

It follows from (4.16) that

$$
u_{j}(-\infty, t)=u_{j}(\infty, t)
$$


Furthermore for the family $J=\left\{J_{t}\right\}_{0 \leq t \leq 1}$ with

$$
J_{t}:=\left(\phi_{H}^{t}\right)_{*}\left(J_{t}^{\prime}\right),
$$

the $u_{j}$ 's satisfy the perturbed Cauchy-Riemann equation (4.12).

We note that (4.13) and the exponential convergence of $u_{j}(\tau)$ to $u_{j}( \pm \infty)$, as $\tau \rightarrow \pm \infty$ respectively, allows us to compactify the maps $u_{j}$ and consider each of them as a cycle defined over a torus $T^{2}$. Therefore the integral $\int u_{j}^{*} \omega$ depends only on the homology class of the compactified cycles.

Now, because $v_{j}: \mathbb{R} \times[0,1] \rightarrow M$ uniformly converges to the constant map $p \in$ Fix $\phi$, the image of $u_{j}$ will be contained in a tubular neighborhood of the closed orbit $z_{H}^{p}: S^{1} \rightarrow M$ of $\dot{x}=X_{H}(x)$ given by

$$
z_{H}^{p}(t)=\phi_{H}^{t}(p) .
$$

In particular, $\int u_{j}^{*} \omega=0$ because the cycle $\left[u_{j}\right]$ is homologous to the one dimensional cycle $\left[z_{H}^{p}\right]$. Then Lemma 4.6 implies the energy $E_{J}\left(u_{j}\right)=0$. But by the choice of $J$ above, (2.31) implies $E_{J^{\prime}}\left(v_{j}\right)=0$, a contradiction to the hypothesis that $v_{j}$ are non-constant. This finishes the proof of Proposition 4.7.

We then define

$$
A\left(\phi, J_{0} ; J^{\prime}\right)=\min \left\{A_{S}\left(\phi, J_{0} ; J^{\prime}\right), A_{D}\left(\phi, J_{0} ; J^{\prime}\right)\right\}
$$

Proposition 4.4 and 4.6 immediately imply

$$
A\left(\phi, J_{0} ; J^{\prime}\right)>0
$$

However the finiteness $A\left(\phi, J_{0} ; J^{\prime}\right)<\infty$ is a nontrivial consequence of Theorem 5.4 below. Finally we define

$$
A\left(\phi, J_{0}\right):=\sup _{J^{\prime} \in j_{\left(\phi, J_{0}\right)}} A\left(\phi, J_{0} ; J^{\prime}\right)
$$

and

$$
A(\phi)=\sup _{J_{0}} A\left(\phi, J_{0}\right) .
$$

By definition, we have $A\left(\phi, J_{0}\right)>0$ and so we have $A(\phi)>0$. However a priori it is not obvious whether they are finite, which will be a consequence of the fundamental existence theorem, Theorem 5.4, in the next section.

\section{$\S 5$. Proof of nondegeneracy}

With the definitions and preliminary studies of the invariants $A\left(\phi, J_{0} ; J^{\prime}\right)$ in section 4 , this section is occupied with the proof of nondegeneracy of the seminorm

$$
\gamma: \operatorname{Ham}(M, \omega) \rightarrow \mathbb{R}_{+}
$$

defined in section 3 . As we pointed out in the introduction, nondegeneracy follows from the following theorem. 
Theorem 5.1. Suppose that $\phi$ is nondegenerate. Then for any $J_{0}$ and $J^{\prime} \in j_{\left(\phi, J_{0}\right)}$, we have

$$
\gamma(\phi) \geq A\left(\phi, J_{0} ; J^{\prime}\right)
$$

and hence

$$
\gamma(\phi) \geq A(\phi)
$$

In particular, $A(\phi)$ is finite.

The rest of this section is occupied by the proof of this theorem.

Let $\phi$ be a nondegenerate Hamiltonian diffeomorphism with $\phi \neq i d$. By the definition of $\gamma$, for any given $\delta>0$, we can find $H \mapsto \phi$ such that

$$
\rho(H ; 1)+\rho(\bar{H} ; 1) \leq \gamma(\phi)+\delta .
$$

For any Hamiltonian $H \mapsto$ know that $\bar{H} \mapsto \phi^{-1}$. We will however use another Hamiltonian

$$
\widetilde{H}(t, x):=-H(1-t, x)
$$

generating $\phi^{-1}$, which is more useful than $\bar{H}$, at least in the study of duality and pants product.

Lemma 5.2. Let $H \mapsto \phi$. Then $\widetilde{H} \mapsto \phi^{-1},\left[\phi^{-1}, \bar{H}\right]=\left[\phi^{-1}, \widetilde{H}\right]$. In addition, if the (nondegenerate) spectrality axiom holds for $a=1$, then we have

$$
\rho(\bar{H} ; 1)=\rho(\widetilde{H} ; 1) .
$$

Proof. A direct calculation shows that the Hamiltonian path generated by $\widetilde{H}$ is given by the path

$$
t \mapsto \phi_{H}^{(1-t)} \circ\left(\phi_{H}\right)^{-1} .
$$

The following composition of homotopies shows that this path is homotopic to the path $t \mapsto\left(\phi_{H}^{t}\right)^{-1}$. Consider the homotopy

$$
\phi_{s}^{t}:= \begin{cases}\phi_{H}^{s-t} \circ\left(\phi_{H}^{s}\right)^{-1} & \text { for } 0 \leq t \leq s \\ \left(\phi_{H}^{t}\right)^{-1} & \text { for } s \leq t \leq 1\end{cases}
$$

It is easy to check that the Hamiltonian path for $s=1$ is (5.6) and the one for $s=0$ is $t \mapsto\left(\phi_{H}^{t}\right)^{-1}$ and satisfies $\phi_{s}^{1} \equiv\left(\phi_{H}\right)^{-1}$ for all $s \in[0,1]$. This finishes the proof of $\left[\phi^{-1}, \bar{H}\right]=\left[\phi^{-1}, \widetilde{H}\right]$. Now (5.5) follows from the homotopy invariance, Theorem 2.12 .

Lemma 5.2 now allows us to write $\gamma$ also as

$$
\gamma(\phi)=\inf _{H \mapsto \phi}(\rho(H ; 1)+\rho(\widetilde{H} ; 1)) .
$$

One advantage of using the representative $\widetilde{H}$ over $\bar{H}$ is that the time reversal

$$
t \mapsto 1-t
$$


acting on the loops $z: S^{1} \rightarrow M$ induces a natural one-one correspondence between $\operatorname{Crit}(H)$ and $\operatorname{Crit}(\widetilde{H})$. Furthermore the space-time reversal

$$
(\tau, t) \mapsto(-\tau, 1-t)
$$

acting on the maps $u: \mathbb{R} \times S^{1} \rightarrow M$ induces a bijection between the moduli spaces $\mathcal{M}(H, J)$ and $\mathcal{M}(\widetilde{H}, \widetilde{J})$ of the perturbed Cauchy-Riemann equations corresponding to $(H, J)$ and $(\widetilde{H}, \widetilde{J})$ respectively, where $\widetilde{J}_{t}=J_{1-t}$. This correspondence reverses the flow and the corresponding actions satisfy

$$
\mathcal{A}_{\widetilde{H}}([\widetilde{z}, \widetilde{w}])=-\mathcal{A}_{H}([z, w])
$$

Here $[\widetilde{z}, \widetilde{w}]$ is the class corresponding to $\widetilde{z}(t):=z(1-t)$ and $\widetilde{w}=w \circ c$ where $c: D^{2} \rightarrow D^{2}$ is the complex conjugation of $D^{2} \subset \mathbb{C}$.

The following estimate of the action difference is an important ingredient in our proof of nondegeneracy. The proof here is similar to the analogous non-triviality proof for the Lagrangian submanifolds studied in [§6-7, Oh4].

Proposition 5.3. Let $J_{0}$ be any compatible almost complex structure, $J^{\prime} \in j_{\left(\phi, J_{0}\right)}$ and $J$ be the one-periodic family $J_{t}=\left(\phi_{H}^{t}\right)_{*} J_{t}^{\prime}$. Let $H$ be any Hamiltonian with $H \mapsto \phi$. Consider the equation

$$
\left\{\begin{array}{l}
\frac{\partial u}{\partial \tau}+J_{t}\left(\frac{\partial u}{\partial t}-X_{H}(u)\right)=0 \\
u(-\infty)=\left[z_{-}, w_{-}\right], u(\infty)=\left[z_{+}, w_{+}\right] \\
w_{-} \# u \sim w_{+}, \quad u(0,0)=q \in B(\lambda)
\end{array}\right.
$$

for a map $u: \mathbb{R} \times S^{1} \rightarrow M$. If (5.9) has a broken trajectory solution (without sphere bubbles attached)

$$
u_{1} \# u_{2} \# \cdots \cdots \# u_{N}
$$

which is a connected union of solutions of (5.9) for $H$ that satisfies the asymptotic condition

$$
\begin{aligned}
& u_{N}(\infty)=\left[z^{\prime}, w^{\prime}\right], u_{1}(-\infty)=[z, w] \\
& u_{j}(0,0)=q \text { for some } j .
\end{aligned}
$$

For some $[z, w] \in \operatorname{Crit} \mathcal{A}_{H}$ and $\left[\widetilde{z}^{\prime}, \widetilde{w}^{\prime}\right] \in \operatorname{Crit} \mathcal{A}_{\widetilde{H}}$, then we have

$$
\mathcal{A}_{H}(u(-\infty))-\mathcal{A}_{H}(u(\infty)) \geq A_{D}\left(\phi, J_{0} ; J^{\prime}\right) .
$$

Proof. Suppose $u$ is such a solution. Opening up $u$ along $t=0$, we define a map $v: \mathbb{R} \times[0,1] \rightarrow M$ by

$$
v(\tau, t)=\left(\phi_{H}^{t}\right)^{-1}(u(\tau, t)) .
$$

It is straightforward to check that $v$ satisfies (4.7) as explained in subsection 2.4. Moreover we have

$$
\int\left|\frac{\partial v}{\partial \tau}\right|_{J_{t}^{\prime}}^{2}=\int\left|\frac{\partial u}{\partial \tau}\right|_{J_{t}}^{2}<\infty
$$


from the energy identity (2.31). Since $\phi(B(\lambda)) \cap B(\lambda)=\emptyset$, we have

$$
v( \pm \infty) \in \operatorname{Fix} \phi \subset M \backslash B(\lambda) .
$$

On the other hand since $v(0,0)=u(0,0) \in B(\lambda), v$ cannot be a constant map. In particular, we have

$$
\int\left|\frac{\partial v}{\partial \tau}\right|_{J_{t}^{\prime}}^{2}=\int v^{*} \omega \geq A_{D}\left(\phi, J_{0} ; J^{\prime}\right)
$$

Combining (5.12) and (5.13), we have proven

$$
\mathcal{A}_{H}(u(-\infty))-\mathcal{A}_{H}(u(\infty))=\int\left|\frac{\partial u}{\partial \tau}\right|_{J_{t}}^{2} \geq A_{D}\left(\phi, J_{0} ; J^{\prime}\right)
$$

This finishes the proof.

This proposition demonstrates relevance of the existence result of the equation (5.9) to Theorem 5.1. However we still need to control the asymptotic condition (5.10) and establish some relevance of the asymptotic condition to the inequality (5.1). For this, we use (5.8) and impose the asymptotic condition

$$
u(-\infty)=[z, w], u(\infty)=\left[z^{\prime}, w^{\prime}\right]
$$

so that

$$
[z, w] \in \alpha_{H}, \quad\left[\widetilde{z}^{\prime}, \widetilde{w}^{\prime}\right] \in \beta_{\widetilde{H}}
$$

for the suitably chosen fundamental Floer cycles $\alpha_{H}$ of $H$ and $\beta_{\widetilde{H}}$ of $\widetilde{H}$.

We recall from (5.3) and (5.5) that we have

$$
\rho(H ; 1)+\rho(\widetilde{H} ; 1) \leq \gamma(\phi)+\delta .
$$

By the definition (2.24) of $\rho$, there exist Floer cycles $\alpha_{H} \in C F_{n}(H)$ and $\beta_{\widetilde{H}} \in$ $C F_{n}(\widetilde{H})$ representing $1^{b}=[M]$ such that

$$
\begin{aligned}
& \rho(H ; 1) \leq \lambda_{H}\left(\alpha_{H}\right) \leq \rho(H ; 1)+\frac{\delta}{2} \\
& \rho(\widetilde{H} ; 1) \leq \lambda_{\widetilde{H}}\left(\beta_{\widetilde{H}}\right) \leq \rho(\widetilde{H} ; 1)+\frac{\delta}{2} .
\end{aligned}
$$

Once we have these, by adding the two, we obtain

$$
\begin{aligned}
0 \leq \rho(H ; 1)+\rho(\widetilde{H} ; 1) & \leq \lambda_{H}\left(\alpha_{H}\right)+\lambda_{\widetilde{H}}\left(\beta_{\widetilde{H}}\right) \\
& \leq \rho(H ; 1)+\rho(\widetilde{H} ; 1)+\delta .
\end{aligned}
$$

The fundamental cycles $\alpha_{H}$ and $\beta_{\widetilde{H}}$ that satisfy (5.15) will be used as the asymptotic boundary condition required in (5.14).

The following is the fundamental existence theorem of the Floer trajectory with its asymptotic limits lying near the 'top' of the given Floer fundamental cycles which will make the difference

$$
\mathcal{A}_{H}([z, w])-\mathcal{A}_{H}\left(\left[z^{\prime}, w^{\prime}\right]\right)=\mathcal{A}_{H}([z, w])+\mathcal{A}_{\widetilde{H}}\left(\left[\widetilde{z}^{\prime}, \widetilde{w}^{\prime}\right]\right)
$$

as close to $\rho(H ; 1)+\rho(\widetilde{H} ; 1)$ as possible. We refer readers to section 8 for its proof. 
Theorem 5.4 [Fundamental Existence Theorem]. Let $\phi, H$ and $\left(J_{0} ; J^{\prime}\right)$ be as in Proposition 5.3 and let $q \in M \backslash \operatorname{Fix}(\phi)$ be given. Choose $\delta$ so that

$$
0<\delta<A_{D}\left(\phi, J_{0} ; J^{\prime}\right) .
$$

Then there exist some fundamental cycles $\alpha_{H}$ of $(H, J)$ for $J_{t}=\left(\phi_{H}^{t}\right)_{*} J_{t}^{\prime}$, and $\beta_{\widetilde{H}}$ of $(\widetilde{H}, \widetilde{J})$ such that they satisfy

$$
\begin{aligned}
& \lambda_{H}\left(\alpha_{H}\right) \leq \rho(H ; 1)+\frac{\delta}{2} \\
& \lambda_{\widetilde{H}}\left(\beta_{\widetilde{H}}\right) \leq \rho(\widetilde{H} ; 1)+\frac{\delta}{2}
\end{aligned}
$$

and have some generators $[z, w] \in \alpha_{H}$ and $\left[\widetilde{z}^{\prime}, \widetilde{w}^{\prime}\right] \in \beta_{\widetilde{H}}$ that satisfy the following alternative :

(1) (5.9) has a broken-trajectory solution (without sphere bubbles attached)

$$
u_{1} \# u_{2} \# \cdots \cdots \# u_{N}
$$

which is a connected union of Floer trajectories for $H$ that satisfies the asymptotic condition

$$
u_{N}(\infty)=\left[z^{\prime}, w^{\prime}\right], u_{1}(-\infty)=[z, w], \quad u_{j}(0,0)=q \in B(\lambda)
$$

for some $1 \leq j \leq N$, and hence

$$
\mathcal{A}_{H}([z, w])-\mathcal{A}_{H}\left(\left[z^{\prime}, w^{\prime}\right]\right) \geq A_{D}\left(\phi, J_{0} ; J^{\prime}\right)
$$

from Proposition 5.3) or,

(2) there exists a $J_{t}^{\prime}$-holomorphic sphere $v_{\infty}: S^{2} \rightarrow M$ for some $t \in[0,1]$ passing through the point $q \in B(\lambda)$, and hence

$$
\mathcal{A}_{H}([z, w])-\mathcal{A}_{H}\left(\left[z^{\prime}, w^{\prime}\right]\right) \geq A_{S}\left(\phi, J_{0} ; J^{\prime}\right)-\delta .
$$

This in particular implies

$$
A\left(\phi, J_{0} ; J^{\prime}\right)<\rho(H ; 1)+\rho(\widetilde{H} ; 1)+\delta<\infty
$$

for any $J^{\prime}$ and $J_{0}$.

Wrap-up of the proof of nondegeneracy. Let $\phi$ be a nondegenerate Hamiltonian diffeomorphism. From the definition of $\gamma(\phi)$, since $\delta$ and $H$ are arbitrary as long as $H \mapsto \phi$, we immediately derive, from (5.21),

$$
A\left(\phi, J_{0} ; J^{\prime}\right) \leq \gamma(\phi)
$$

for all $J_{0}$ and $J^{\prime} \in j_{\left(\phi, J_{0}\right)}$. Next by taking the supremum of $A\left(\phi, J_{0} ; J^{\prime}\right)$ over all $J_{0}$ and $J^{\prime} \in j_{\left(\phi, J_{0}\right)}$ in $(5.22)$, we also derive

$$
A(\phi) \leq \gamma(\phi) .
$$

This finishes the proof of Theorem 5.1 and so the proof of nondegeneracy. 


\section{$\S 6$. The spectral norm, the ball area and the homological area of $\phi$}

We first start with improving the lower bound (5.1). For this purpose, we reexamine our set-up of the definition

$$
\gamma(\phi)=\inf _{H \mapsto \phi}(\rho(H ; 1)+\rho(\bar{H} ; 1))=\inf _{H \mapsto \phi}(\rho(H ; 1)+\rho(\widetilde{H} ; 1)) .
$$

This involves only the class $1 \in Q H^{*}(M)$ and the orbits $[z, w]$ of $H$ and $\left[\widetilde{z}^{\prime}, \widetilde{w}^{\prime}\right]$ of $\widetilde{H}$ of Conley-Zehnder indices

$$
\mu_{H}([z, w])=n, \quad \mu_{\widetilde{H}}\left(\left[\widetilde{z}^{\prime}, \widetilde{w}^{\prime}\right]\right)=n .
$$

Remark 6.1. If we reverse the flow of $\widetilde{H}$ by considering the time reversal map

$$
\left[\widetilde{z}^{\prime}, \widetilde{w}^{\prime}\right] \mapsto\left[z^{\prime}, w^{\prime}\right] ; \text { Crit } \mathcal{A}_{\widetilde{H}} \rightarrow \text { Crit } \mathcal{A}_{H}
$$

then the broken-trajectory solution of $u$ constructed in Theorem 5.4 satisfies the asymptotic condition

$$
\begin{aligned}
u(-\infty) & =[z, w] \in C F_{n}(H) \quad \text { i.e., } \mu_{H}([z, w])=n \\
u(\infty) & =\left[z^{\prime}, w^{\prime}\right] \in C F_{-n}(H) \quad \text { i.e., } \mu_{H}\left(\left[z^{\prime}, w^{\prime}\right]\right)=-n
\end{aligned}
$$

with respect to our grading convention. We emphasize that the time reversal map also replaces the downward Novikov ring with the upward Novikov ring, which is a source of complications in the non-aspherical case.

Let $\phi$ be nondegenerate. We now introduce a stronger lower bound of $\gamma(\phi$, denoted by $A(\phi ; 1)$. This is a refined version of $A(\phi)$ which correctly reflects the fact that the definition of $\gamma$ involves only the class $1 \in Q H^{*}(M)$.

Let $J_{0}$ be a given almost complex structure and $J^{\prime} \in j_{\left(\phi, J_{0}\right)}$. Fix a point $q \in$ $M \backslash$ Fix $\phi$. Let $\alpha_{H}$ and $\beta_{\widetilde{H}}$ be the Floer Novikov cycles defined in section 5 with $\left[\alpha_{H}\right]=\left[\beta_{\widetilde{H}}\right]=1^{b}=[M]$. Consider all broken-trajectory solutions

$$
u=u_{1} \# u_{2} \# \cdots \# u_{N}
$$

where each $u_{k}$ itself is a cusp-curve which has the unique principal component with a finite number of sphere bubbles. Furthermore the chain of principal components satisfies

$$
\left\{\begin{array}{l}
\frac{\partial u}{\partial \tau}+J_{t}\left(\frac{\partial u}{\partial t}-X_{H}(u)\right)=0 \\
u_{1}(-\infty)=[z, w] \in \alpha_{H}, u_{N}(\infty)=\left[z^{\prime}, w^{\prime}\right] \text { with }\left[\widetilde{z}^{\prime}, \widetilde{w}^{\prime}\right] \in \beta_{\widetilde{H}} \\
u_{j}(0,0)=q \text { for some } j=1, \cdots, N
\end{array}\right.
$$

with $J_{t}=\left(\phi_{H}^{t}\right)_{*} J_{t}^{\prime}$. Here one should regard $(0,0)$ as the marked point in the domain of the broken-trajectory curves as a one-marked 'Floer-stable trajectory' in the standard sense of [FOn], [Ru], [LT].

We define the energy of the broken-trajectory $u=u_{1} \# \cdots \# u_{N}$ by

$$
E_{J}(u):=\sum_{k=1}^{N} E_{J}\left(u_{k}\right)
$$


as usual. Here we define $E_{J}\left(u_{k}\right)$ by the sum of the energy of the principal component and the symplectic areas of the sphere bubbles. We would like to recall that there is no loss of energy under the bubbling process and that the symplectic area of such a $J_{t}$-holomorphic sphere bubble has the same value as the harmonic energy with respect to the Riemannian metric associated to $g_{J_{t}}=\omega\left(\cdot, J_{t} \cdot\right)$.

Now we introduce the main definition of this section. Let $J^{\prime} \in j_{\left(\phi, J_{0}\right)}, q \in$ $M \backslash \operatorname{Fix} \phi$ and $\alpha_{H} \in C F_{n}(H), \beta_{\widetilde{H}} \in C F_{n}(\widetilde{H})$ be as above. Opening up $u$ along $t=0 \equiv 1$, we consider

$$
v(\tau, t)=\left(\phi_{H}^{t}\right)^{-1}(u(\tau, t))
$$

on $\mathbb{R} \times[0,1]$ and extend to $\mathbb{R} \times \mathbb{R}$ by

$$
\phi(v(\tau, t+1))=v(\tau, t)
$$

as before. We define

$$
\begin{gathered}
A\left(\phi, J_{0} ; J^{\prime} ; \alpha_{H}, \beta_{\widetilde{H}} ; q\right)=\inf _{u}\left\{E_{J}(u) \mid u \text { satisfies and }(6.3), u(0,0)=q\right\} \\
=\inf _{v}\left\{\int v^{*} \omega \mid v \text { satisfies }(1.14)\right. \text { with } \\
\exists\left[z^{-}, w^{-}\right] \in \alpha_{H}: z^{-}(t)=\phi_{H}^{t}(v(-\infty)) \\
\exists\left[\widetilde{z}^{+}, \widetilde{w}^{+}\right] \in \beta_{\widetilde{H}}: z^{+}(t)=\phi_{H}^{t}(v(+\infty)) \\
\text { and } v(0,0)=q\} .
\end{gathered}
$$

As usual, we set $A\left(\phi, J_{0} ; J^{\prime} ; \alpha_{H}, \beta_{\widetilde{H}} ; q\right)=\infty$, if (6.3) has no solution. By definition, we have the upper bound

$$
A\left(\phi, J_{0} ; J^{\prime} ; \alpha_{H}, \beta_{\widetilde{H}} ; q\right) \leq \lambda_{H}\left(\alpha_{H}\right)+\lambda_{\widetilde{H}}\left(\beta_{\widetilde{H}}\right)
$$

if (6.3) has a solution. We then set

$$
\begin{aligned}
A\left(\phi, J_{0} ; J^{\prime} ; 1 ; q\right) & =\inf _{\alpha_{H}, \beta_{\widetilde{H}}}\left\{A\left(\phi, J_{0} ; J^{\prime} ; \alpha_{H}, \beta_{\widetilde{H}} ; q\right) \mid\left[\alpha_{H}\right]=1^{b}=\left[\beta_{\widetilde{H}}\right]\right\} . \\
A\left(\phi, J_{0} ; J^{\prime} ; 1\right) & =\sup _{q \in M \backslash \operatorname{Fix} \phi} A\left(\phi, J_{0} ; J^{\prime} ; 1 ; q\right) .
\end{aligned}
$$

Theorem 5.4 implies

$$
A\left(\phi, J_{0} ; J^{\prime} ; 1 ; q\right) \leq \rho(H ; 1)+\rho(\widetilde{H} ; 1)=\gamma(H)
$$

for all $q \in M \backslash \operatorname{Fix} \phi$, and hence

$$
A\left(\phi, J_{0} ; J^{\prime} ; 1\right) \leq \gamma(H) .
$$

By definition, we also have

$$
A\left(\phi, J_{0} ; J^{\prime} ; 1\right) \geq A\left(\phi, J_{0} ; J^{\prime}\right) .
$$

Finally we give the main definition. 
Definition 6.2 (Homological area). Let $\phi, J_{0}$ and $J^{\prime}$ as above. We define

$$
A\left(\phi, J_{0} ; 1\right)=\sup _{J^{\prime} \in j_{\left(\phi, J_{0}\right)}} A\left(\phi, J_{0} ; J^{\prime} ; 1\right),
$$

and

$$
A(\phi ; 1)=\sup _{J_{0}} A\left(\phi, J_{0} ; 1\right) .
$$

We call $A(\phi ; 1)$ the homological area of $\phi$.

With this definition, we have obtained the following theorem.

Theorem 6.3. Let $\phi$ be nondegenerate, and $J_{0}$ and $J^{\prime} \in j_{\left(\phi, J_{0}\right)}$ as before. Then we have

$$
A\left(\phi, J_{0} ; J^{\prime} ; 1\right) \leq \gamma(\phi)
$$

for all $J_{0}$ and $J^{\prime} \in j_{\left(\phi, J_{0}\right)}$. In particular, we have

$$
0<A(\phi ; 1) \leq \gamma(\phi)<\infty .
$$

Proof. Let $H \mapsto \phi$. We take the supremum of the right hand side of (6.6) over all $H \mapsto \phi$ which gives rise to $(6.7)$. This proves that $A\left(\phi, J_{0} ; J^{\prime} ; 1\right)$ is finite. We then take the infimum of $(6.6)$ over all $\left(J_{0} ; J^{\prime}\right)$, which gives rise to the proof of $(6.8)$.

Motivated by Theorem 6.3, we introduce the following new capacities.

Definition 6.4. Let $B$ be any compact subset of $M$.

(1) (Spectral displacement energy) We define

$$
e_{\gamma}(B)=\inf _{\phi}\{\gamma(\phi) \mid \phi(B) \cap B=\emptyset\}
$$

and call it the spectral displacement energy.

(2) (Homological area capacity) We define

$$
c_{\text {hom }}(B):=\inf _{\phi}\{A(\phi ; 1) \mid \phi(B) \cap B=\emptyset\}
$$

and call $c_{\text {hom }}(B)$ the homological area capacity of $B$. As usual, we say $c_{\text {hom }}(B)=\infty$ if $B$ cannot be displaced from itself by any Hamiltonian diffeomorphism.

With these definitions, the following lower bound of the spectral displacement energy $e_{\gamma}(B)$ immediately follows from Theorem 6.3.

Theorem 6.5. For any compact set $B \subset M$, we have

$$
e_{\gamma}(B) \geq c_{h o m}(B) \text {. }
$$

Now, to demonstrate that $A(\phi ; 1)$ is a much finer invariant than $A(\phi)$, we provide an example of $\phi$ for which we have the strict inequality

$$
0<A(\phi)<A(\phi ; 1)
$$

on $S^{2}$, and a deformation of $\phi$ converging to a degenerate Hamiltonian diffeomorphism such that $A(\phi) \rightarrow 0$ while $A(\phi ; 1)$ is fixed. 
Example 6.6. Consider the unit sphere $S^{2}$ with the standard area form of area $4 \pi$, and let $f$ be a Morse function such that $-f$ has the unique maximum point $p^{+}$ and the minimum point $p^{-}$such that

$$
\max f-\min f=f\left(p^{+}\right)-f\left(p^{-}\right)<2 \pi, \quad \int_{S^{2}} f d \mu=0 .
$$

In addition, we make $f$ to have a pair consisting of local maximum point $p_{1}$ and a saddle point $p_{2}$ such that there exists a unique gradient trajectory connecting $p_{2}$ and $p_{1}$. We can make the difference $(-f)\left(p_{1}\right)-(-f)\left(p_{2}\right)=f\left(p_{2}\right)-f\left(p_{1}\right)>0$ as small as we want. Now we consider the autonomous Hamiltonian flow $\phi_{f}^{t}$ of $f$ and set $\phi=\phi_{f}^{1}$. Then it is easy to see that the Morse complex of $-f$ is given by

$$
\partial^{(-f)} p^{+}=-p_{2}, \partial^{(-f)} p_{1}=p_{2}, \partial^{(-f)} p_{2}=0, \partial^{(-f)} p^{-}=0
$$

where $\partial^{(-f)}$ is the boundary map in the Morse complex of $-f$. Therefore we have

$$
\begin{aligned}
& \operatorname{ker} \partial^{(-f)}=\operatorname{span}_{\mathbb{Z}}\left\{p^{+}+p_{1}, p^{-}, p_{2}\right\} \\
& \operatorname{im} \partial^{(-f)}=\operatorname{span}_{\mathbb{Z}}\left\{p_{2}\right\} .
\end{aligned}
$$

Hence we have the Morse homology of $f$ given by

$$
H M_{2}(-f)=\left\{\left[p^{+}+p^{1}\right]\right\}, \quad H M_{0}(-f)=\left\{\left[p^{-}\right]\right\}, \quad H M_{1}(-f)=\{0\} .
$$

We claim

$$
\begin{aligned}
A(\phi ; 1) & =\max f-\min f=f(p+)-f\left(p^{-}\right) \\
A(\phi) & =f\left(p_{1}\right)-f\left(p_{2}\right)
\end{aligned}
$$

and in particular we have $0<A(\phi)<A(\phi ; 1)$.

By considering $f$ whose $C^{2}$-norm is sufficiently small, the Floer complex with respect to the standard complex structure $J_{0}=j$ is given by

$$
(C F(f), \partial) \cong\left(C M(-f), \partial^{(-f)}\right) \otimes \Lambda .
$$

It immediately follows that if we consider $J^{\prime}=\left(\phi_{f}^{t}\right)^{*} J_{0}$, then $J^{\prime} \in j_{\left(\phi, J_{0}\right)}$ and the maps

$$
v_{f}(\tau, t)=\phi_{f}^{t}(\chi(\tau))
$$

are solutions of the mapping cylinder version of the Cauchy-Riemann equation

$$
\left\{\begin{array}{l}
\frac{\partial v}{\partial \tau}+J_{t}^{\prime} \frac{\partial v}{\partial t}=0 \\
\phi(v(\tau, 1))=v(\tau, 0), \quad \int_{\mathbb{R} \times[0,1]}\left|\frac{\partial v}{\partial \tau}\right|_{J_{t}^{\prime}}^{2}<\infty
\end{array}\right.
$$

for any gradient trajectory $\chi: \mathbb{R} \rightarrow M$ satisfying

$$
\dot{\chi}-\operatorname{grad} f(\chi)=0 .
$$

In particular the unique gradient trajectory connecting $p_{1}, p_{2}$ gives rise to the unique Floer trajectory of (6.14) with the same asymptotic condition. It is easy to check that this trajectory is Fredholm regular. Therefore by the cobordism 
argument, there exists at least one solution of (6.14) with the same area as $v_{f}$ for all (generic) choices of $J_{0}$ and $J^{\prime} \in j_{\left(\phi, J_{0}\right)}$. This proves

$$
A\left(\phi, J_{0} ; J^{\prime}\right)=f\left(p_{1}\right)-f\left(p_{2}\right)
$$

for all such $J_{0}, J^{\prime}$ which in particular proves

$$
A(\phi)=f\left(p_{1}\right)-f\left(p_{2}\right) .
$$

Next since we have the fundamental class $1^{b}=\left[p^{+}+p^{1}\right]$, it follows that for each given point $p \in S^{2} \backslash K$ where $K$ is the union of the trajectories connecting

$$
K=\bar{W}\left(p^{+}, p_{2}\right) \cup \bar{W}\left(p_{1}, p_{2}\right) \cup \bar{W}\left(p_{2}, p^{-}\right)
$$

$p^{+}, p_{1}$ to $p_{2}$ and $p^{2}$ to $p^{-}$, there exists a unique trajectory passing through $p$, issued at one of the local maximum points $\left\{p^{+}, p_{1}\right\}$ to $p^{-}$by the cap action in the Morse homology of $-f$. (See [Oh4] for a proof of this fact.) Here we denote by $W(p, q)$ the intersection

$$
W(p, q)=W^{-}(p) \cap W^{+}(q)
$$

and by $\bar{W}(p, q)$ its closure, where $W^{-}(p), W^{+}(q)$ denote the unstable and stable manifolds of $p$ and $q$ respectively. Note that the closure $\bar{W}\left(p_{2}, p^{-}\right)$divides $S^{2}$ into two components

$$
S^{2} \backslash \bar{W}\left(p_{2}, p^{-}\right)=\operatorname{Int} W\left(p^{+}\right) \coprod \operatorname{Int} W\left(p_{1}\right) .
$$

Now if we choose $p$ from Int $W\left(p^{+}\right)$, then the corresponding $v_{f}$ has area given by $\max f-\min f=f\left(p^{+}\right)-f\left(p^{-}\right)$.

It will be shown in section 10 that as long as the $C^{2}$ norm of $f$ is sufficiently small, the 'thin' part of the Floer moduli space is separated from the 'thick' part thereof. This then implies, again by the cobordism argument, that there exists at least one Floer trajectory connecting $p^{+}$and $p^{-}$for all generic choice of $J_{0}$ and $J^{\prime} \in j_{\left(\phi, J_{0}\right)}$ with area given by $f\left(p^{+}\right)-f\left(p^{-}\right)$. This then proves

$$
A(\phi ; 1)=f\left(p^{+}\right)-f\left(p^{-}\right) .
$$

Now (6.15) and (6.16) prove our claim.

Finally, by deforming $f$ so that it remains unchanged outside a neighborhood of $\bar{W}\left(p_{1}, p_{2}\right)$ but letting the value $f\left(p_{1}\right)-f\left(p_{2}\right) \rightarrow 0$ (see [Figure 5.2, Mi] for the description of such a deformation of Morse functions), we also see that $A(\phi)$ with $\phi=\phi_{f}$ can go to zero as $\phi$ converges to a degenerate Hamiltonian diffeomorphism, which is not the identity, while $A(\phi ; 1)$ is fixed.

The remaining section will be occupied by some discussion on the relation between (6.11) and the optimal energy-capacity inequality for the Hofer displacement energy $e(B) \geq c(B)$. Recall that the Gromov capacity $c(B)$ is defined by

$$
\begin{aligned}
c(B)= & \sup _{\lambda}\left\{\lambda=\pi r^{2} \mid g\left(B^{2 n}(r)\right) \subset \operatorname{Int} B,\right. \\
& \left.g: B^{2 n}(r) \rightarrow(M, \omega) \text { is a symplectic embedding }\right\} .
\end{aligned}
$$

Another examination of the proof of Theorem 6.1 suggests the following general conjecture 
[The Area Conjecture].

(1) Let $\phi$ be a nondegenerate Hamiltonian diffeomorphism. Then we have

$$
A(\phi ; 1) \geq \operatorname{bArea}(\phi) .
$$

(2) The function $\phi \mapsto A(\phi ; 1)$ is continuous on $\operatorname{Ham}^{\text {nd }}(M, \omega)$ (in the $C^{\infty}$ topology), and can be continuously extended to the $\operatorname{set} \operatorname{Ham}(M, \omega)$ as a continuous function. In particular, the inequality (6.17) holds for any arbitrary Hamiltonian diffeomorphism.

Combining Theorem 6.3 and the Area Conjecture, and using the continuity of $\gamma$ and bArea on $\operatorname{Ham}(M, \omega)$ under the $C^{\infty}$-topology, we now state several conjectures which are immediate consequences of the Area Conjecture and Theorem 6.5.

Conjecture 6.7. Let $\phi$ be any Hamiltonian diffeomorphism. Then we have

$$
\gamma(\phi) \geq \operatorname{bArea}(\phi) .
$$

Conjecture 6.8. Let $B \subset M$ be a closed subset. Then we have $c_{\text {hom }}(B) \geq c(B)$.

We note that we have the inequality

$$
e_{\gamma}(B) \leq e(B)
$$

where $e(B)$ is the Hofer displacement energy

$$
e(B)=\inf _{\phi}\{\|\phi\| \mid \phi(B) \cap B=\emptyset\} .
$$

An immediate corollary of Theorem 6.5 and Conjecture 6.8 would be the optimal energy-capacity inequality for the Hofer displacement energy $e(B) \geq c(B)$. We hope to carry out further study of the homological area $A(\phi ; 1)$ and the Area Conjecture elsewhere in the future.

\section{$\S 7$. The homological area and the generating function of $\phi$}

In this section, we will obtain some lower estimates, in the spirit of [BP, Mc], for the Hofer norm of Hamiltonian diffeomorphisms whose graphs in $(M,-\omega) \times(M, \omega)$ allow generating functions in the Darboux chart of the diagonal $\Delta \subset M \times M$. As an application, we will prove Theorem IV and its corollary stated in the introduction.

Suppose that the Hamiltonian diffeomorphism $\phi$ has the property that its graph

$$
\Delta_{\phi}:=\operatorname{graph} \phi \subset(M,-\omega) \times(M, \omega)
$$

is close to the diagonal $\Delta \subset M \times M$ in the following sense: Let $o_{\Delta}$ be the zero section of $T^{*} \Delta$ and

$$
\Phi:(\mathcal{U}, \Delta) \subset M \times M \rightarrow\left(\mathcal{V}, o_{\Delta}\right) \subset T^{*} \Delta
$$

be a Darboux chart such that

(1) $\Phi^{*} \omega_{0}=-\omega \oplus \omega$ and

(2) $\left.\Phi\right|_{\Delta}=i d_{\Delta}$ and $\left.d \Phi\right|_{T \mathcal{U}_{\Delta}}:\left.\left.T \mathcal{U}\right|_{\Delta} \rightarrow T \mathcal{V}\right|_{o_{\Delta}}$ is the obvious symplectic bundle map from $T(M \times M)_{\Delta} \cong N \Delta \oplus T \Delta$ to $T\left(T^{*} \Delta\right)_{o_{\Delta}} \cong T^{*} \Delta \oplus T \Delta$ which is the identity on $T \Delta$ and the canonical map

$$
\left.\widetilde{\omega}: N \Delta \rightarrow T^{*} \Delta ; \quad X \mapsto X\right\rfloor \omega
$$

on the normal bundle $N \Delta=T(M \times M)_{\Delta} / T \Delta$. 
Consider the path

$$
t \mapsto \operatorname{graph} t d S_{\phi}
$$

which is a Hamiltonian isotopy of the zero section and define $\phi^{t}: M \rightarrow M$ to be the Hamiltonian diffeomorphism satisfying

$$
\Phi\left(\Delta_{\phi^{t}}\right)=\operatorname{graph} t d S_{\phi}
$$

for $0 \leq t \leq 1$. By the requirement (1) and (2) for the Darboux chart $\Phi$ of (7.1), it follows that the path $t \mapsto \phi^{t}$ is a Hofer geodesic, i.e., a quasi-autonomous Hamiltonian path: We recall a Hamiltonian path $t \mapsto \phi^{t}$ is called quasi-autonomous [BP], if its generating Hamiltonian $H$ has a fixed maximum and a fixed minimum point over $t \in[0,1]$. In addition, we note that the Hamiltonian path $\phi^{t}$ provided by (7.2) has no non-constant one periodic orbits at all. We denote by $H=H^{\phi}$ the corresponding Hamiltonian with $\phi^{t}=\phi_{H^{\phi}}^{t}$.

We recall

$$
\rho(H ; 1)+\rho(\bar{H} ; 1) \leq \int_{0}^{1}\left(\max H_{t}-\min H_{t}\right) d t
$$

On the other hand, if $H$ is the one obtained from (7.2), we also have

$$
\int_{0}^{t}-\min H_{u} d u=t \max S_{\phi}, \quad \int_{0}^{t}-\max H_{u} d u=t \min S_{\phi} .
$$

and so

$$
\gamma(\phi) \leq \rho(H ; 1)+\rho(\bar{H} ; 1) \leq \max S_{\phi}-\min S_{\phi}:=\operatorname{osc}\left(S_{\phi}\right) .
$$

With this definition, we will prove the following theorem in section 9 , as a consequence of an existence theorem for solutions of (1.14) satisfying the asymptotic conditions required in (6.4), with the lower energy estimate.

All the results in the rest of this section can be generalized for arbitrary engulfable Hamiltonian diffeomorphisms. For the simplicity of exposition, we will restrict to the case where $\phi$ is $C^{1}$-close to the identity, leaving the generalizations to the engulfable case in [Oh10].

The following is the main theorem of this section. We will prove the theorem in section 9 as a consequence of an existence theorem of 'thin' solutions of (1.14).

Theorem 7.1. Let $\phi$ be sufficiently $C^{1}$-small, and $S_{\phi}$ be as above. Then we have

$$
A(\phi ; 1) \geq \operatorname{osc}\left(S_{\phi}\right) \text {. }
$$

Assuming this for the moment, we state several consequences of the theorem. First we state the following corollary.

Corollary 7.2. Let $\phi$ be a $C^{1}$-small Hamiltonian diffeomorphism and $S_{\phi}$ be its generating function as defined above. Then we have

$$
\gamma(\phi)=\operatorname{osc}\left(S_{\phi}\right)=A(\phi ; 1) .
$$

Proof. Combining (6.8), (7.5) and (7.6), we derive the following chain of inequalities

$$
A(\phi ; 1) \leq \gamma(\phi) \leq \operatorname{osc}\left(S_{\phi}\right) \leq A(\phi ; 1) .
$$

Therefore all the inequalities here are indeed the equalities. This finishes the proof.

We now derive several consequences from this Corollary 7.2. 
Theorem 7.3. Let $\Phi: \mathcal{U} \rightarrow \mathcal{V}$ be a Darboux chart along the diagonal $\Delta \subset M \times M$, and $H=H^{\phi}$ is the Hamiltonian generating $\phi^{t}: M \rightarrow M$ as in (7.2). Then the path $t \in[0,1] \rightarrow \phi_{H}^{t}$ is length minimizing among all paths from the identity to $\phi$. In this case, we also have $\gamma(\phi)=\|\phi\|$.

Proof. Let $K \mapsto \phi$ and $\phi_{K}^{t}$ be the corresponding Hamiltonian path. By the canonical adjustment [Lemma 9.2, Oh5], we may assume that $K$ is one-periodic. It follows from (3.2) that

$$
\gamma(K) \leq\|K\|
$$

Combining Corollary 7.2 and (7.4)-(7.5), we have obtained

$$
\left\|H^{\phi}\right\|=\operatorname{osc}\left(S_{\phi}\right)=A(\phi, 1)=\gamma(\phi) .
$$

In particular, we have

$$
\gamma(\phi)=\left\|H^{\phi}\right\|
$$

Therefore we derive $\left\|H^{\phi}\right\| \leq\|K\|$ from (7.8) and (7.9) which is exactly what we wanted to prove. The identity $\gamma(\phi)=\|\phi\|$ follows from $\gamma(\phi) \leq\|\phi\|,(7.9)$ and the inequality $\left\|H^{\phi}\right\| \geq\|\phi\|$ by the definition of $\|\phi\|$. This finishes the proof.

As in [BP, Mc], an immediate corollary of Theorem 7.3 is the following $C^{1}$ flatness result of $\operatorname{Ham}(M, \omega)$.

Corollary 7.4 [Proposition 1.8, Mc]. There is a path connected neighborhood

$$
\mathcal{N} \subset \operatorname{Ham}(M, \omega)
$$

of the identity in the $C^{1}$-topology such that any element in $\mathcal{N}$ can be joined to the identity by a path that minimizes the Hofer length. Moreover $(\mathcal{N},\|\cdot\|)$ is isometric to a neighborhood of $\{0\}$ in a normed vector space, which is nothing but the space $C_{m}^{\infty}(M)$ of normalized functions on $M$ with the norm given by osc $(f)=$ $\max f-\min f$.

\section{PART II: ADIABATIC DEGENERATION AND THICK-THIN DECOMPOSITION}

\section{$\S 8$. Proof of the fundamental existence theorem}

In this section, we will give a complete self-contained proof of Theorem 5.4, modulo some background materials presented in [En1], [Oh9], restricting to the case when there is no discontinuity in the Hamiltonian perturbation term and when the associated Hamiltonian fibration is 'flat'.

\subsection{Pants product and Hamiltonian fibrations.}

We first recall the description of conformal structures on a compact Riemann surface $\Sigma$ of genus 0 with three punctures in terms of the minimal area metric [Z] which will be important for the analysis involving the adiabatic degeneration of pseudo-holomorphic curves relevant to the pants product.

We conformally identify $\Sigma$ with the union of three half cylinders which we denote by $\Sigma_{1}, \Sigma_{2}$ and $\Sigma_{3}$ in the following way: the conformal structure on $\Sigma \backslash\left\{x_{1}, x_{2}, x_{3}\right\}$ can be described in terms of the minimal area metric $[\mathrm{Z}]$ which we denote by $g_{\Sigma}$. 
This metric makes $\Sigma$ the union of three half-cylinders $\Sigma_{i}$ with flat metric with each meridian circle having length 1 . The glued metric on $\Sigma$ is smooth everywhere except at two points $p, \bar{p} \in \Sigma$ lying on the boundary circles of $\Sigma_{i}$. As in [FOh], we will use this metric for the analytic estimates of pseudo-holomorphic curves implicit in the argument. One important property of this singular metric is that the metric is flat everywhere except at the two points $p, \bar{p}$ where the metric has a conical singularity. Therefore its associated conformal structure smoothly extends across the singularities $p, \bar{p}$. (See [St] for such a removable singularity theorem of the conformal structure.) The resulting conformal structure is the standard unique conformal structure on $\Sigma=S^{2} \backslash\{0,1, \infty\}$.

As in [FOh], [En1], we identify $\Sigma$ as the union of $\Sigma_{i}$ 's

$$
\Sigma=\cup_{i=1}^{3} \Sigma_{i}
$$

in the following way: if we identify $\Sigma_{i}$ with $(-\infty, 0] \times S^{1}$, then there are 3 paths $\theta_{i}$ of length $\frac{1}{2}$ for $i=1,2,3$ in $\Sigma$ connecting $p$ to $\bar{p}$ such that

$$
\begin{aligned}
& \partial_{1} \Sigma=\theta_{1} \circ \theta_{3}^{-1} \\
& \partial_{2} \Sigma=\theta_{2} \circ \theta_{1}^{-1} \\
& \partial_{3} \Sigma=\theta_{3} \circ \theta_{2}^{-1} .
\end{aligned}
$$

We fix a holomorphic identification of each $\Sigma_{i}, i=1,2$ with $(-\infty, 0] \times S^{1}$ and $\Sigma_{3}$ with $[0, \infty) \times S^{1}$ considering the decomposition (8.1).

We denote the identification by

$$
\varphi_{i}^{+}: \Sigma_{i} \rightarrow(-\infty, 0] \times S^{1}, \quad i=1,2
$$

for positive punctures and

$$
\varphi_{3}^{-}: \Sigma_{3} \rightarrow[0, \infty) \times S^{1}
$$

for the negative puncture. We denote by $(\tau, t)$ the standard cylindrical coordinates on the cylinders.

We fix a cut-off function $\rho_{-}:(-\infty, 0] \rightarrow[0,1]$ of the type

$$
\rho_{-}= \begin{cases}1 & \text { for } \tau \leq-r_{\text {rig }} \\ 0 & \text { for }-r_{\text {lef }} \leq \tau \geq 0\end{cases}
$$

with

$$
0<r_{\text {lef }}<r_{\text {rig }}
$$

and define $\rho_{+}:[0, \infty) \rightarrow[0,1]$ by $\rho_{+}(\tau)=\rho_{-}(-\tau)$. We just denote by $\rho$ these cut-off functions when there is no danger of confusion.

We now consider the (topologically) trivial smooth bundle $P \rightarrow \Sigma$ with fiber isomorphic to $(M, \omega)$ and fix a trivialization

$$
\Phi_{i}: P_{i}:=\left.P\right|_{\Sigma_{i}} \rightarrow \Sigma_{i} \times M
$$

on each $\Sigma_{i}$ for $i=1,23$. On each $P_{i}$, we consider the closed two form of the type

$$
\omega_{P_{i}}:=\Phi_{i}^{*}\left(\omega+d\left(\rho H_{t} d t\right)\right)
$$


for a time periodic Hamiltonian $H:[0,1] \times M \rightarrow \mathbb{R}$. Note that this naturally extends to a closed two form $\omega_{P}$ defined on the whole $P \rightarrow \Sigma$ since the cut-off functions vanish in the center region of $\Sigma$. This form is nondegenerate in the fiber and restricts to $\Phi_{i}^{*} \omega$.

Such $\omega_{P}$ is called a coupling form and induces a canonical symplectic connection $\nabla=\nabla_{\omega_{P}}$ [GLS], [En1]. In addition it also fixes a natural deformation class of symplectic forms on $P$ obtained by those

$$
\Omega_{P, \lambda}:=\omega_{P}+\lambda \omega_{\Sigma}
$$

where $\omega_{\Sigma}$ is an area form and $\lambda>0$ is a sufficiently large constant. We will always normalize $\omega_{\Sigma}$ so that $\int_{\Sigma} \omega_{\Sigma}=1$.

Next let $\widetilde{J}$ be an almost complex structure on $P$ such that

(1) $\widetilde{J}$ is $\omega_{P}$-compatible on each fiber and so preserves the vertical tangent space and

(2) the projection $\pi: P \rightarrow \Sigma$ is pseudo-holomorphic, i.e, $d \pi \circ \widetilde{J}=j \circ d \pi$.

When we are given three $t$-periodic Hamiltonians $H=\left(H_{1}, H_{2}, H_{3}\right)$ and $J=$ $\left(J_{1}, J_{2}, J_{3}\right)$, we say that $\widetilde{J}$ is $(H, J)$-compatible, if $\widetilde{J}$ additionally satisfies

(3) For each $i,\left(\Phi_{i}\right)_{*} \widetilde{J}=j \oplus J_{H_{i}}$ where

$$
J_{H_{i}}(\tau, t, x)=\left(\phi_{H_{i}}^{t}\right)^{*} J_{i}
$$

on $M$ over a disc $D_{i}^{\prime} \subset D_{i}$ in terms of the cylindrical coordinates. Here $D_{i}^{\prime}=$ $\varphi_{i}^{-1}\left(\left(-\infty,-r_{i, r i g}\right] \times S^{1}\right), i=1,2$ and $\varphi_{3}^{-1}\left(\left[r_{3, \text { rig }}, \infty\right) \times S^{1}\right)$ for some $r_{i, \text { rig }}>0$.

Condition (3) implies that the $\widetilde{J}$-holomorphic sections $s$ over $\Sigma_{i}^{\prime}$ for $i=1,23$ are precisely the solutions of the equation

$$
\frac{\partial u}{\partial \tau}+J_{t}^{i}\left(\frac{\partial u}{\partial t}-X_{H_{i}}(u)\right)=0
$$

if we write

$$
\Phi_{i}(s(\tau, t))=\left(\tau, t, u_{i}(\tau, t)\right)
$$

in the trivialization with respect to the cylindrical coordinates $(\tau, t)$ on $D_{i}^{\prime}$ induced by $\phi_{i}^{ \pm}$above.

Now we are ready to define the moduli space which will be relevant to the definition of the pants product that we need to use. To simplify the notation, we denote

$$
\widehat{z}=[z, w]
$$

in general and $\widehat{z}=\left(\widehat{z}_{1}, \widehat{z}_{2}, \widehat{z}_{3}\right)$ where $\widehat{z}_{i}=\left[z_{i}, w_{i}\right] \in \operatorname{Crit} \mathcal{A}_{H_{i}}$ for $i=1,2,3$.

Definition 8.1. Consider the pair $(H, J)$ of $H=\left\{H_{i}\right\}_{1 \leq i \leq 3}$ and $J=\left\{J_{i}\right\}_{1 \leq i \leq 3}$, and let $\widetilde{J}$ be a $(H, J)$-compatible almost complex structure. Define $\mathcal{M}(H, \widetilde{J} ; \widetilde{z})$ to be the space of all $\widetilde{J}$-holomorphic sections $s: \Sigma \rightarrow P$ that satisfy

(1) The maps $u_{i}:\left(-\infty,-r_{i, r i g}\right] \times S^{1} \rightarrow M$ which are solutions of (8.4), satisfy

$$
\lim _{\tau \rightarrow-\infty} u_{i}(\tau, \cdot)=z_{i}, \quad i=1,2
$$

where $\Phi_{i}(s(\tau, t))=\left(\tau, t, u_{i}(\tau, t)\right)$, and similarly for $i=3$ changing $-\infty$ to $+\infty$.

(2) The closed surface obtained by capping off $p r_{M} \circ \Phi_{i}(s(\Sigma))$ with discs $w_{i}$ taken with the same orientation for $i=1,2$ and the opposite orientation for $i=3$ represents zero in $\pi_{2}(M)$. 
We denote by $\widetilde{\pi}_{2}(M)$ the set of elements in $\pi_{2}(M)$ modulo $\mathbb{Z}$-torsion elements.

Note that $\mathcal{M}(H, \widetilde{J} ; \widehat{z})$ depends only on the equivalence class of $\widehat{z}$ : we say that $\widehat{z}^{\prime} \sim \widehat{z}$ if

$$
z_{i}^{\prime}=z_{i}, \quad w_{i}^{\prime}=w_{i} \# A_{i}
$$

for $A_{i} \in \pi_{2}(M)$ and $\sum_{i=1}^{3} A_{i}$ represents zero in $\widetilde{\pi}_{2}(M)$. The dimension of $\mathcal{M}(H, \widetilde{J} ; \widehat{z})$ is given by

$$
\begin{aligned}
\operatorname{dim} \mathcal{M}(H, \widetilde{J} ; \widehat{z}) & =2 n-\left(-\mu_{H_{1}}\left(z_{1}\right)+n\right)-\left(-\mu_{H_{2}}\left(z_{2}\right)+n\right)-\left(\mu_{H_{3}}\left(z_{3}\right)+n\right) \\
& =-n+\left(-\mu_{H_{3}}\left(z_{3}\right)+\mu_{H_{1}}\left(z_{1}\right)+\mu_{H_{2}}\left(z_{2}\right)\right)
\end{aligned}
$$

Note that when $\operatorname{dim} \mathcal{M}(H, \widetilde{J} ; \widehat{z})=0$, we have

$$
\mu_{H_{3}}\left(\widehat{z}_{3}\right)=\mu_{H_{1}}\left(\widehat{z}_{1}\right)+\mu_{H_{2}}\left(\widehat{z}_{2}\right)-n \text {. }
$$

Now the pair-of-pants product $*$ for chains is defined by

$$
\widehat{z}_{1} * \widehat{z}_{2}=\sum_{\widehat{z}_{3}} \#(\mathcal{M}(H, \widetilde{J} ; \widehat{z})) \widehat{z}_{3}
$$

for the generators $\widehat{z}_{i}$ and then by linearly extending over the chains in $C F_{*}\left(H_{1}\right) \otimes$ $C F_{*}\left(H_{2}\right)$. Our grading convention makes this product have degree $-n$.

From now on, we will specialize our discussion to the triple $\left(H_{1}, H_{2}, H_{3}\right)$ that allows a coupling form $\omega_{P}$ whose associated symplectic connection becomes flat. For example, the triple with $H_{3}=H_{1} \# H_{2}$ is one such choice. For such a 'flat' connection on $P \rightarrow \Sigma$, we have the identity

$$
\frac{1}{2} \int_{\Sigma}\left|(D s)^{v}\right|_{\widetilde{J}}^{2}=\int s^{*} \omega_{P}
$$

for any $(H, J)$-compatible $\widetilde{J}$. In general, such a flat connection must have some pointwise singularities and it is not possible to construct a smooth connection that is flat everywhere, but one can choose a smooth connection whose curvature becomes arbitrarily small both pointwise and in the integral sense. We refer readers to [section $7, \mathrm{Oh} 9]$ or to (10.9)-(10.10) coming later for the $L^{2}$-estimates of the derivative $|D s|_{\widetilde{J}}$ in terms of the curvature of the general Hamiltonian connection. We decompose $D s=(D s)^{v}+(D s)^{h}$ the sum of the vertical and horizontal parts with respect to the chosen connection. Then we have the identity

$$
\int_{\Sigma} s^{*} \omega_{P}=\mathcal{A}_{H_{1}}\left(\left[z_{1}, w_{1}\right]\right)+\mathcal{A}_{H_{2}}\left(\left[z_{2}, w_{2}\right]\right)-\mathcal{A}_{H_{1} \# H_{2}}\left(\left[z_{3}, w_{3}\right]\right)
$$

for any $\widetilde{J}$-holomorphic sections $s \in \mathcal{M}(H, \widetilde{J} ; \widehat{z})$. We refer to [ $\S 5$, En1] or [Sc] for its proof. We note that this, combined with the integral bounds on the curvature, provides the uniform (total) energy bound for the pseudo-holomorphic sections $s \in \mathcal{M}(H, \widetilde{J} ; \widehat{z})$. (See (10.9).) Therefore we will mainly concern the vertical part of the energy. 
Then (8.7) and (8.8) give rise to

$$
\frac{1}{2} \int_{\Sigma}\left|(D s)^{v}\right|_{\widetilde{J}}^{2}=-\mathcal{A}_{H_{1} \# H_{2}}\left(\left[z_{3}, w_{3}\right]\right)+\mathcal{A}_{H_{1}}\left(\left[z_{1}, w_{1}\right]\right)+\mathcal{A}_{H_{2}}\left(\left[z_{2}, w_{2}\right]\right)
$$

if we choose the above mentioned singular flat connection. Otherwise, (8.9) will be only an approximate identity whose error can be made arbitrarily small. We will ignore this small error for the rest of discussion, preferring to use the singular connection that is flat everywhere except at two points, $p, \bar{p}$. However we emphasize that the bundle $P \rightarrow \Sigma$ itself is smooth.

This general identity can be also used the other way around. More precisely, the following lower bound will be a crucial ingredient for the limiting arguments for the pseudo-holomorphic sections with some asymptotic conditions which are allowed to vary inside given Floer homology classes. The proof of this lemma is an immediate consequence of (8.9) and the energy estimate [Corollary 4.4, Oh9] which is omitted.

Lemma 8.2. Let $\widehat{z}_{i}=\left[z_{i}, w_{i}\right]$ for $i=1,2$, 3. Suppose $\mathcal{A}_{H_{3}}\left(\left[z_{3}, w_{3}\right]\right) \geq c$ for some constant $c$. Then we have the lower bound

$$
\mathcal{A}_{H_{1}}\left(\left[z_{1}, w_{1}\right]\right)+\mathcal{A}_{H_{2}}\left(\left[z_{2}, w_{2}\right]\right) \geq c-\left(E^{+}\left(H_{1}\right)+E^{+}\left(H_{2}\right)\right)+E^{-}\left(H_{3}\right) .
$$

We will apply the above discussion to the triple

$$
H_{1}=H, H_{2}=\widetilde{H} \#(\epsilon f), H_{3}=\epsilon f
$$

for which a flat connection can be explicitly constructed. We give the construction of such a connection in Appendix 1.

Now (8.8) becomes

$$
\frac{1}{2} \int_{\Sigma}\left|(D s)^{v}\right|_{\widetilde{J}}^{2}=-\mathcal{A}_{\epsilon f}([q, \widehat{q}])+\mathcal{A}_{H}\left(\left[z_{1}, w_{1}\right]\right)+\mathcal{A}_{\widetilde{H} \#(\epsilon f)}\left(\left[z_{2}, w_{2}\right]\right) .
$$

\subsection{Heuristic discussion of the proof of Theorem 5.4.}

To motivate our proof of Theorem 5.4, we first give the following heuristic discussion imitating the proof of a similar existence theorem given by the author [section $7, \mathrm{Oh} 4]$ for the purpose of proving nondegeneracy of the invariant defined by (1.12) on the cotangent bundle. We refer to [Oh7] for more detailed explanations.

Morally we would like to apply the pants product to the case

$$
H_{1}=H, H_{2}=\widetilde{H}, H_{3}=0
$$

for a pseudo-holomorphic section of an appropriate Hamiltonian bundle $P \rightarrow \Sigma$ with boundary data

$$
\begin{aligned}
& \left.\Phi \circ s\right|_{\partial_{1} \Sigma}=[z, w] \in \alpha_{H},\left.\Phi \circ s\right|_{\partial_{2} \Sigma}=\left[\widetilde{z}^{\prime}, \widetilde{w}^{\prime}\right] \in \beta_{\widetilde{H}} \\
& \left.\Phi \circ s\right|_{\partial_{3} \Sigma}=[q, \widehat{q}] \in[M]
\end{aligned}
$$

with

$$
\mathcal{A}_{H}([z, w])+\mathcal{A}_{\widetilde{H}}\left(\left[\widetilde{z}^{\prime}, \widetilde{w}^{\prime}\right]\right) \geq C(H),
$$


where $C(H)$ is a constant depending only on $H$. (See [Corollary 4.4, Oh9] for the precise value of $C(H)$.)

Note that because $H_{3}=0$ in the monodromy condition (8.13) and the outgoing end with monodromy $H_{2}=\widetilde{H}$ is equivalent to the incoming end with monodromy $H_{2}=H$, we can fill-up the hole $z_{3} \in \Sigma$ and consider the cylinder with one outgoing and one incoming end with the same monodromy $H_{1}=H$. In other words our Hamiltonian bundle $P \rightarrow \Sigma$ becomes the mapping cylinder

$$
E_{\phi}:=\mathbb{R} \times \mathbb{R} \times M /(\tau, t+1, \phi(x)) \sim(\tau, t, x) \rightarrow \mathbb{R} \times S^{1}
$$

of $\phi$ with the canonical Hamiltonian connection $\nabla_{H}$ induced by $H$. (See [En1] for a detailed explanation of the relation between Hamiltonians and connections.) If we make the almost complex structure $\widetilde{J}$ the pushforward of the complex structure

$$
\widetilde{J}(\tau, t, x)=j \oplus J^{\prime}(t, x)
$$

on $\mathbb{R} \times \mathbb{R} \times M$ under the covering projection

$$
\pi: \mathbb{R} \times \mathbb{R} \times M \rightarrow E_{\phi}=(\mathbb{R} \times \mathbb{R} \times M) /(\tau, t+1, \phi(x)) \sim(\tau, t, x)
$$

and the natural connection induced by the Hamiltonian $H:[0,1] \times \mathbb{R} \rightarrow M$, which is flat, then $\widetilde{J}$-pseudo-holomorphic sections of $E_{\phi}$ become solutions of the perturbed Cauchy-Riemann equation for $H$. The condition $\left.\Phi \circ s\right|_{\partial_{3} \Sigma}=[q, \widehat{q}]$ in (8.14) leads to the condition $q \in \operatorname{Im} u$. Now the identity $1 * 1=1$ would lead to such a solution which in turn would prove $\gamma(\phi)>0$ via Proposition 5.3.

However in reality, $H_{3}=0$ is a degenerate Hamiltonian and cannot be directly used for the construction of the above pants product moduli space, which is the reason we have to consider (8.11) instead and take the limiting argument as $\epsilon \rightarrow 0$. This requires us to study the singular degeneration problem, the so called adiabatic degeneration, of the type studied in [FOh] but with nontrivial quantum contributions around.

\subsection{Construction of solutions I: analysis of the thick part.}

In this subsection, we start the proof of the main existence result, Theorem 5.4.

Suppose that we are given any $J^{\prime} \in j_{\left(\phi, J_{0}\right)}$, and one fixed $J_{3}^{\prime} \in j_{\left(\phi_{\epsilon f}^{1}, J_{0}\right)}$. Let $\left(\phi, J_{0}\right)$ be as before and $f$ be a generic Morse function. For the sake of simplicity, we will also assume that $f$ has a unique minimum point.

We consider the admissible triple

$$
H_{1}=H, H_{2}=\widetilde{H} \#(\epsilon f), H_{3}=\epsilon f
$$

for the nondegenerate $H$. We note that if $H$ is nondegenerate and $\epsilon$ is sufficiently small, $H \#(\epsilon f)$ is also nondegenerate and there is a natural one-one correspondence between $\operatorname{Per}(H)$ and $\operatorname{Per}(H \#(\epsilon f))$ and the critical points $\operatorname{Crit} \mathcal{A}_{H}$ and $\operatorname{Crit} \mathcal{A}_{\widetilde{H} \#(\epsilon f)}$. We again denote by $\left[z^{\prime}, w^{\prime}\right]$ the critical point of $\mathcal{A}_{H \#(\epsilon f)}$ corresponding to $\left[\widetilde{z}^{\prime}, \widetilde{w}^{\prime}\right] \in$ $\operatorname{Crit} \mathcal{A}_{\widetilde{H} \#(\epsilon f)} \cdot$

Then we consider the triple of the families of almost complex structures $J=$ $\left(J_{1}, J_{2}, J_{3}\right)$ on the three ends $\Sigma_{i} \cong\left(-\infty,-r_{i, \text { rig }}\right] \times S^{1}$ under the trivialization

$$
\Phi_{i}:\left.P\right|_{\Sigma_{i}} \rightarrow \Sigma_{i} \times M
$$


defined by

$$
\begin{aligned}
J_{t}^{1} & =\left(\phi_{H}^{t}\right)_{*} J_{t}^{\prime}, \\
J_{t}^{2} & =\left(\phi_{\widetilde{H} \#(\epsilon f)}^{t}\right)_{*}\left(\phi_{-\epsilon f}^{1-t}\right)^{*} J_{(1-t)}^{\prime} \quad \text { and } \\
J_{t}^{3} & =\left(\phi_{\epsilon f}^{t}\right)_{*} J_{3, t}^{\prime}
\end{aligned}
$$

respectively. Here the precise forms of $J_{t}^{i}$ are not important except the point that they are chosen to be one-periodic. Note that the family $\left(\phi_{-\epsilon f}^{1-t}\right)^{*} J_{(1-t)}^{\prime} \in$ $j_{\left(\phi_{\widetilde{H} \#(\epsilon f)}, J_{0}\right)}$ and $J^{2}$ is still a one-periodic family. As $\epsilon \rightarrow 0, J^{2}$ converges to the time-reversal family of $t \mapsto\left(\phi_{H}^{t}\right)_{*} J_{t}^{\prime}$

$$
t \mapsto\left(\phi_{H}^{(1-t)}\right)_{*} J_{(1-t)}^{\prime} .
$$

We also choose $J_{3, t}^{\prime}$ so that

$$
J_{t}^{3} \equiv J_{0}
$$

at the end of $\Sigma_{3}$ and the path converges to the constant path $J_{0}$ as $\epsilon \rightarrow 0$. This is possible since $\phi_{\epsilon f}^{1}$ converges to the identity map.

With these choices made, we study the limit as $\epsilon \rightarrow 0$ of the moduli space

$$
\mathcal{M}\left(H^{\epsilon}, \widetilde{J}^{\epsilon} ; \widehat{z}^{\epsilon}\right)
$$

with the asymptotic boundary condition (8.14). The main difficulty in studying this limit problem is that the limit is a singular limit and it is possible that the image of the pseudo-holomorphic curves can collapse to an object of Hausdorff dimension one. Our first non-trivial task is to prove that there is a suitable limiting procedure for any sequence $s^{\epsilon} \in \mathcal{M}\left(H^{\epsilon}, \widetilde{J}^{\epsilon} ; \widehat{z}^{\epsilon}\right)$ as $\epsilon \rightarrow 0$. We will show that there exists a sub-sequence of $s^{\epsilon} \in \mathcal{M}\left(H^{\epsilon}, \widetilde{J}^{\epsilon} ; \widehat{z}^{\epsilon}\right)$ that converge to the union

$$
u \cup \chi
$$

in the Hausdorff topology, where $\chi$ is a negative (cusp) gradient trajectory of $-f$ landing at the critical point $q \in$ Crit $f$ and $u$ is a solution of (5.9) but satisfying

$$
u(0,0) \in \operatorname{Im} \chi
$$

instead of $u(0,0)=q$. In particular, we will prove that the piece of Hausdorff dimension one is always the image of a (negative) gradient trajectory of $-f$.

This proven, it is easy to see that the above mentioned heuristic discussion cannot produce a solution required in Theorem 5.4 unless the negative gradient trajectory of $-f$ landing at $q$ is trivial for some reason. At this stage, the condition

$$
\mu_{-\epsilon f}^{\text {Morse }}(q)=2 n
$$

will play an essential role and enable us to conclude that the only gradient trajectories of $-f$ landing at $q$ are the constant map $q$ and so we are in a good position to start with. 
Let $P \rightarrow \Sigma, \omega_{P}$ and $\Omega_{P, \lambda}$ be those as defined in subsection 7.1. We equip $P$ with an $(H, J)$-compatible almost complex structure $\widetilde{J}$ such that

$$
\widetilde{J}=j \oplus J_{i}^{\prime}
$$

on each $\Sigma_{i}$ where

$$
J_{1}^{\prime} \in j_{\left(\phi, J_{0}\right)}, \quad J_{2}^{\prime}=\widetilde{J}_{1}^{\prime} \in j_{\left(\phi^{-1}, J_{0}\right)}, \quad J_{3}^{\prime} \in j_{\left(\phi_{\epsilon f}^{1}, J_{0}\right)} .
$$

More explicitly we define $\widetilde{J}$ by

$$
\widetilde{J}(\tau, t, x)(\alpha, \beta, \xi)=\left(-\beta, \alpha,\left(\phi_{H_{i}^{\rho(\tau)}}^{t}\right)^{*} J_{i, t}\left(\xi-\beta X_{\rho(\tau) H_{i}}\right)+\alpha X_{\rho(\tau) H}\right)
$$

on each $\Sigma_{i}$ for each $i=1,2,3$. (See [Oh9] for detailed usage of the choice (8.16) in the derivation of $L^{2}$-bounds of the derivative $D s$ for a $\widetilde{J}$-holomorphic section $s$ of $P$.)

The $\widetilde{J}$-holomorphic sections $s$ over $\Sigma_{i}$ are precisely the solutions of the equation

$$
\frac{\partial u}{\partial \tau}+J_{t}^{i}\left(\frac{\partial u}{\partial t}-X_{H_{i}}(u)\right)=0
$$

if we write $\Phi_{i}(s(\tau, t))=\left(\tau, t, u_{i}(\tau, t)\right)$ in the trivialization with respect to the cylindrical coordinates $(\tau, t)$ on $\Sigma_{i}^{\prime}$ induced by $\varphi_{i}^{ \pm}$as before.

By the construction, we have the identity

$$
1_{\epsilon f}^{b}=h_{i d}\left((1 \cdot 1)^{b}\right)=[\alpha * \beta]
$$

in homology for any cycles $\alpha \in C F_{*}(H), \beta \in C F_{*}(\widetilde{H} \#(\epsilon f))$ with

$$
[\alpha]=1^{b},[\beta]=1^{b},[\gamma]=1^{b},
$$

. In the level of cycles, we have

$$
\alpha * \beta=\gamma+\partial_{\epsilon f}(\eta)
$$

for some $\eta \in C F_{*}(\epsilon f)$. We recall the following fundamental tightness result of the classical Mores fundamental cycle in the Floer complex $C F_{*}(\epsilon f)$.

Proposition 8.3 [Lemma 8.8, Oh5]. Consider the autonomous Hamiltonian $\epsilon f$ and the cohomology class $1 \in Q H^{0}(M) \cong H Q^{2 n}(-\epsilon f)$. Then there is a unique Novikov cycle $\gamma$ of $\epsilon f$ of the form

$$
\gamma=\sum_{j} c_{j}\left[x_{j}, \widehat{x}_{j}\right]
$$

with $x_{j} \in$ Crit $_{2 n}(-\epsilon f)$ that represents the class $1^{b}=[M]$. Furthermore the cycle $\gamma$ is tight in the sense of Definition 2.13: for any Novikov cycle $\beta$ of $\epsilon f$ in the class $[\beta]=[M]$, i.e, $\beta$ homologous to $\gamma$, we have

$$
\lambda_{\epsilon f}(\beta) \geq \lambda_{\epsilon f}(\gamma)
$$


It follows from Proposition 8.3 and (8.18) that

$$
\lambda_{\epsilon f}(\alpha * \beta) \geq-\frac{\delta}{3}
$$

with $\delta$ independent of $\epsilon$ by choosing $\epsilon$ sufficiently small for any cycles $\alpha \in C F_{*}(H)$ and $\beta \in C F_{*}(\widetilde{H} \#(\epsilon f))$.

Now we specialize the above discussion to particularly chosen $\alpha$ and $\beta$. By the definition of $\rho(\cdot ; 1)$, we can find $\alpha_{H} \in C F_{n}(H)$ and $\beta_{\widetilde{H}} \in C F_{n}(\widetilde{H})$ satisfying

$$
\begin{aligned}
& \lambda_{H}\left(\alpha_{H}\right) \leq \rho(H ; 1)+\frac{\delta}{2} \\
& \lambda_{\widetilde{H}}\left(\beta_{\widetilde{H}}\right) \leq \rho(\widetilde{H} ; 1)+\frac{\delta}{2} .
\end{aligned}
$$

We now transfer the cycle $\beta_{\widetilde{H}}$ to a cycle $\beta_{\widetilde{H} \#(\epsilon f)} \in C F_{n}(\widetilde{H} \#(\epsilon f))$ defined by

$$
\beta_{\widetilde{H} \#(\epsilon f)}:=h_{\epsilon}\left(\beta_{\widetilde{H}}\right)
$$

where $h_{\epsilon}: C F_{n}(\widetilde{H}) \rightarrow C F_{n}(\widetilde{H} \#(\epsilon f))$ is the Floer chain map $h_{(\mathcal{L}, j)}$ over the linear homotopy $\mathcal{L}$ between $\widetilde{H}$ and $\widetilde{H} \#(\epsilon f)$ and $j$ close to the constant homotopy $J$. Then we will have

$$
\rho(\widetilde{H} ; 1)-\epsilon \max f \leq \lambda_{\widetilde{H}}\left(\beta_{H}\right) \leq \rho(\widetilde{H} ; 1)+\frac{\delta}{2}+\epsilon(-\min f) .
$$

Here the upper bound immediately follows from (2.17). However to get the lower bound, we use the choice made in (5.17) for $\delta$ to apply the Handle sliding lemma [Oh5], more specifically Corollary 6.4 [Oh5] : By the choice (5.17) of $\delta$, we have

$$
\frac{\delta}{2}+\min (-\epsilon f)<A_{D}\left(\phi, J_{0} ; J^{\prime}\right)\left(=A_{(H, J)}\right)
$$

if $\epsilon$ is sufficiently small. Here $A_{(H, J)}$ is the constant for the equation (4.12) defined similarly as $A_{D}\left(\phi, J_{0} ; J^{\prime}\right)$. This precludes the level of $\beta_{\widetilde{H}}$ going down too much under the chain map $h_{\epsilon}$, because we know

$$
|\rho(\widetilde{H} \#(\epsilon f) ; 1)-\rho(\widetilde{H} ; 1)| \leq \epsilon|f| .
$$

We refer readers to [section6, Oh5] for the details of this argument to prove such a lower bound.

Furthermore by the continuity of $\rho(\cdot ; 1)$, we also have

$$
\lim _{\epsilon \rightarrow 0} \rho(\widetilde{H} \#(\epsilon f) ; 1)=\rho(\widetilde{H} ; 1) .
$$

Now we need to compare the values of $\lambda_{H}\left(\alpha_{H}\right), \lambda_{\widetilde{H} \#(\epsilon f)}\left(\beta_{\widetilde{H} \#(\epsilon f)}\right)$ and $\lambda_{\epsilon f}\left(\alpha_{H} *\right.$ $\left.\beta_{\widetilde{H} \#(\epsilon f)}\right)$.

For each given $\epsilon>0$, let $\left[z_{\epsilon}, w_{\epsilon}\right] \in \alpha_{H}$ and $\left[\widetilde{z}_{\epsilon}^{\prime}, \widetilde{w}_{\epsilon}^{\prime}\right] \in \beta_{\widetilde{H} \#(\epsilon f)}$ and $[q, \widehat{q} \# A] \in$ $\gamma+\partial_{\epsilon f}(\eta)$ such that the moduli space $\mathcal{M}\left(H^{\epsilon}, \widetilde{J}^{\epsilon} ; \widetilde{z}^{\epsilon}\right)$ is non-empty. Such a triple 
exists because of the relation (8.18) and by the definition of the pants product ' $*$ '. Using (8.21), we may choose $[q, \widehat{q} \# A] \in \gamma+\partial_{\epsilon f}(\eta)$ so that

$$
\mathcal{A}_{\epsilon f}([q, \widehat{q} \# A]) \geq-\frac{\delta}{2}
$$

Furthermore since $\left[\alpha_{H} * \beta_{\widetilde{H} \#(\epsilon f)}\right]=1^{b}$ and the unique maximum point of $-\epsilon f$ is homologically essential, we may also assume that $q$ is the unique maximum point and $A=0$. For each given $\epsilon>0$, let $s^{\epsilon}$ be any $\widetilde{J}$-holomorphic section of $P \rightarrow \Sigma$ with asymptotic boundary conditions

$$
\begin{aligned}
& \left.s\right|_{\partial_{1} \Sigma}=\left[z_{\epsilon}, w_{\epsilon}\right] \in \alpha_{H},\left.s\right|_{\partial_{2} \Sigma}=\left[\widetilde{z}_{\epsilon}^{\prime}, \widetilde{w}_{\epsilon}^{\prime}\right] \in \beta_{\widetilde{H} \#(\epsilon f)} \\
& \left.s\right|_{\partial_{3} \Sigma}=[q, \widehat{q}] \in \gamma
\end{aligned}
$$

written in the given trivialization $\Phi$. Then it follows from (8.10) of Lemma 8.2 that we have

$$
\mathcal{A}_{H}\left(\left[z_{\epsilon}, w_{\epsilon}\right]\right)+\mathcal{A}_{\widetilde{H} \#(\epsilon f)}\left(\left[\widetilde{z}_{\epsilon}^{\prime}, \widetilde{w}_{\epsilon}^{\prime}\right]\right) \geq-\frac{\delta}{2}-\left(E^{+}(H)+E^{+}(\widetilde{H} \#(\epsilon f))\right)+E^{-}(\epsilon f) .
$$

By choosing $\epsilon>0$ sufficiently small, we may assume that

$$
\mathcal{A}_{H}\left(\left[z_{\epsilon}, w_{\epsilon}\right]\right)+\mathcal{A}_{\widetilde{H} \#(\epsilon f)}\left(\left[\widetilde{z}_{\epsilon}^{\prime}, \widetilde{w}_{\epsilon}^{\prime}\right]\right) \geq-\left(E^{+}(H)+E^{+}((\widetilde{-\epsilon f) \# H}))-\delta^{\prime}:=C(H)\right.
$$

where $\delta^{\prime}$ can be made arbitrarily small by letting $\epsilon \rightarrow 0$. In particular, combining (8.23) and (8.26), we derive the lower bounds

$$
\begin{gathered}
C(H)-\rho(H ; 1) \leq \mathcal{A}_{H}\left(\left[z_{\epsilon}, w_{\epsilon}\right]\right) \\
C(H)-\rho(\widetilde{H} \#(\epsilon f) ; 1) \leq \mathcal{A}_{\widetilde{H} \#(\epsilon f)}\left(\left[z_{\epsilon}, w_{\epsilon}\right]\right)
\end{gathered}
$$

for all $\epsilon>0$ by adjusting $C(H)$ slightly. Therefore for each given $\epsilon>0,(8.27)$ implies that there are only finitely many possible asymptotic periodic orbits among the generators $\left[z_{\epsilon}, w_{\epsilon}\right] \in \alpha_{H}$ and $\left[z_{\epsilon}^{\prime}, w_{\epsilon}^{\prime}\right] \in \beta_{\widetilde{H} \#(\epsilon f)}$ respectively such that the corresponding moduli space

$$
\mathcal{M}\left(H^{\epsilon}, \widetilde{J}^{\epsilon} ; \widehat{z}^{\epsilon}\right)
$$

becomes non-empty for the asymptotic condition (8.25). On the other hand, since we assume that $H$ is generic and nondegenerate, $(-\epsilon f) \# H$ is nondegenerate for all sufficiently small $\epsilon>0$, there is a canonical one-one correspondence between $\operatorname{Crit} \mathcal{A}_{H}$ and Crit $\mathcal{A}_{H \#(\epsilon f)}$. Therefore as we let $\epsilon \rightarrow 0$, we may assume that the asymptotic orbits $\left[z^{\epsilon}, w^{\epsilon}\right] \in \operatorname{Crit} \mathcal{A}_{H}$ converges to $[z, w] \in \operatorname{Crit} \mathcal{A}_{H}$ and in turn $\left[\widetilde{z}_{\epsilon}^{\prime}, \widetilde{w}_{\epsilon}^{\prime}\right] \in \operatorname{Crit} \mathcal{A}_{\widetilde{H} \#(\epsilon f)}$ converges to $\left[\widetilde{z}^{\prime}, \widetilde{w}^{\prime}\right] \in \operatorname{Crit} \mathcal{A}_{\widetilde{H}}$ in the $C^{\infty}$-topology. It remains to show that

$$
\left[\widetilde{z}^{\prime}, \widetilde{w}^{\prime}\right] \in \beta_{\widetilde{H}} .
$$

We now recall the definition (8.22) of the cycles $\beta_{\widetilde{H} \#(\epsilon f)}$. Because a Novikov cycle is generated by an infinite number of generators, we can not say in general that the whole cycle $\beta_{\widetilde{H} \#(\epsilon f)}$ converges to $\beta_{\widetilde{H}}$ because the convergence of the generators may not be uniform. However it is easy to check by the compactness and transversality argument that the generators in $\beta_{\widetilde{H} \#(\epsilon f)}$ above any given level indeed converge to those of $\beta_{\widetilde{H}}$, using the fact that the linear homotopy from $\widetilde{H}$ to $\widetilde{H} \#(\epsilon f)$ converges to the constant homotopy $\widetilde{H}$ as $\epsilon \rightarrow 0$ and considering the corresponding continuity equation $(2.12)$ for the chain map $h_{\epsilon}$. We leave the details to the readers. This proves (8.28). 
Remark 8.4. Here we like to note that convergence of $\left[z^{\epsilon}, w^{\epsilon}\right] \in \operatorname{Crit} \mathcal{A}_{H}$ is an immediate consequence of the action lower bound (8.27) and the Novikov condition of the cycle $\alpha_{H}$, while convergence of $\left[\widetilde{z}_{\epsilon}^{\prime}, \widetilde{w}_{\epsilon}^{\prime}\right] \in \operatorname{Crit} \mathcal{A}_{H \#(\epsilon f)}$ is a consequence of this and the fixed asymptotic condition

$$
\left.s\right|_{\partial_{3} \Sigma}=[q, \widehat{q}] \in \gamma
$$

in the outgoing end through a standard compactness argument on the moduli space $\mathcal{M}\left(H^{\epsilon}, \widetilde{J}^{\epsilon} ; \widehat{z}^{\epsilon}\right)$. The latter is more subtle than the former because the Hamiltonian $(-\epsilon f) \# H$ varies as $\epsilon \rightarrow 0$. Here the energy bound for the sequence $s^{\epsilon}$, convergence of the other ends and the fact that $\left[\widetilde{z}_{\epsilon}^{\prime}, \widetilde{w}_{\epsilon}^{\prime}\right] \in \operatorname{Crit} \mathcal{A}_{H \#(\epsilon f)}$ occurs as the asymptotic condition of the sequence $s^{\epsilon}$, enter the convergence proof in a crucial way.

Having this convergence statement made for the asymptotic orbits, for the simplicity of notations we omit the subscript $\epsilon$ from $\left[z_{\epsilon}, w_{\epsilon}\right]$ and $\left[z_{\epsilon}^{\prime}, w_{\epsilon}^{\prime}\right]$ and denote them by $[z, w]$ and $\left[\widetilde{z}^{\prime}, \widetilde{w}^{\prime}\right]$ from now on. We also note that because the degree of the quantum cohomology class 1 is zero, we have

$$
\mu_{H}([z, w])=\mu_{\widetilde{H} \#(\epsilon f)}\left(\left[\widetilde{z}^{\prime}, \widetilde{w}^{\prime}\right]\right)=\mu_{\epsilon f}([q, \widehat{q}])=n
$$

and so

$$
\mu_{-\epsilon f}^{\text {Morse }}(q)=n+\mu_{\epsilon f}([q, \widehat{q}])=2 n .
$$

In particular, any gradient trajectory $\chi:(K, \infty) \rightarrow M$ of $-\epsilon f$ satisfying

$$
\dot{\chi}-\epsilon \operatorname{grad} f(\chi)=0, \quad \lim _{\tau \rightarrow \infty} \chi(\tau)=q
$$

must be the constant map $\chi \equiv q$, which is exactly what we wanted to have in our heuristic discussion in the beginning of this section.

We recall the uniform energy bound in (8.9) for the vertical energy of the section $s$. Since this bound is uniform for all sufficiently small $\epsilon$ and for any choice of the cut-off functions $\rho_{i}$ with $r_{i, r i g}>r_{i, l e f}$ mentioned in (8.2), we can now carry out the adiabatic convergence argument for $\Sigma_{3}$. To carry out this adiabatic convergence argument, we will conformally change the metric on the base $\Sigma$ of the fibration. We note that the vertical energy of the section $s$ is invariant under the conformal change of the base metric. We will realize this conformal change by a conformal diffeomorphism

$$
\psi_{\epsilon}: S \backslash\{(0,0)\} \rightarrow\left(\Sigma \backslash\left\{x_{1}, x_{2}, x_{3}\right\}, g_{\epsilon}\right)
$$

where $S=\mathbb{R} \times S^{1}$ is the standard flat cylinder and the metric $g_{\epsilon}$ is constructed in a way similar to the minimal area metric, but we change the lengths of the $\theta_{i}$ 's in (8.1) in the following way :

$$
\begin{aligned}
& \text { length } \theta_{1}=1-\frac{\epsilon}{2} \\
& \text { length } \theta_{2}=\text { length } \theta_{3}=\frac{\epsilon}{2} .
\end{aligned}
$$

It is easy to see that we can choose the conformal diffeomorphism $\psi_{\epsilon}$ so that it restricts to a quasi-isometry

$$
\psi_{\epsilon}: S \backslash D(\delta(\epsilon)) \rightarrow\left(\Sigma_{1}^{\epsilon} \cup \Sigma_{2}^{\epsilon}, g_{\epsilon}\right)
$$


and to a conformal diffeomorphism

$$
\psi_{\epsilon}: D(\delta(\epsilon)) \backslash\{0\} \rightarrow\left(\Sigma_{3}, g_{\epsilon}\right)
$$

where $D(\delta)$ is the disc around $(0,0)$ and $\delta(\epsilon) \rightarrow 0$ as $\epsilon \rightarrow 0: \psi_{\epsilon}$ is the inverse of the conformal diffeomorphism

$$
\psi_{\epsilon}^{-1}(r, \theta)=\delta(\epsilon) e^{-2 \pi(r+\sqrt{-1} \theta)} ; \quad[0, \infty) \times S^{1} \rightarrow D(\delta(\epsilon)) \backslash\{0\} .
$$

We choose any sequences $\epsilon_{j} \rightarrow 0$ and a $(H, J)$-compatible $\widetilde{J}^{\epsilon}$. Then choose any sequence $s_{j} \in \mathcal{M}\left(H^{\epsilon_{j}}, \widetilde{J}^{\epsilon} ; \widehat{z}^{\epsilon_{j}}\right)$ that satisfy (8.25). Consider the compositions

$$
\widetilde{s}_{j}=s_{j} \circ \psi_{\epsilon_{j}} ; S \backslash\{(0,0)\} \rightarrow P .
$$

Since $\psi_{\epsilon}$ is conformal, we have

$$
\int\left|\left(D \widetilde{s}_{j}\right)^{v}\right|_{\widetilde{J}^{\epsilon}}^{2}=\int\left|\left(D s_{j}\right)^{v}\right|_{\widetilde{J}^{\epsilon}}^{2}
$$

and hence the uniform energy bound

$$
\begin{aligned}
\frac{1}{2} \int\left|\left(D \widetilde{s}_{j}\right)^{v}\right|_{\widetilde{J}^{\epsilon}}^{2} & =-\mathcal{A}_{\epsilon f}([q, \widehat{q}])+\mathcal{A}_{\widetilde{H} \#(\epsilon f)}\left(\left[\widetilde{z}^{\prime}, \widetilde{w}^{\prime}\right]\right)+\mathcal{A}_{H}([z, w]) \\
& \leq\left(\rho(H ; 1)+\frac{\delta}{2}\right)+\left(\rho(\widetilde{H} \#(\epsilon f))+\frac{\delta}{2}\right)+\frac{\delta}{2}
\end{aligned}
$$

provided $\epsilon$ is sufficiently small. Here we used (8.9) for the identity, (8.23)-(8.24) for the inequality.

After choosing a subsequence, there are two alternatives to consider :

(1) there exists some constant $c>0$ such that

$$
\frac{1}{2} \int_{D^{2}\left(\delta\left(\epsilon_{j}\right)\right) \backslash\{(0,0)\}}\left|\left(D \widetilde{s}_{j}\right)^{v}\right|_{\widetilde{J}^{\epsilon}}^{2}>c .
$$

$(2)$

$$
\limsup _{j \rightarrow \infty} \frac{1}{2} \int_{D^{2}\left(\delta\left(\epsilon_{j}\right)\right) \backslash\{(0,0)\}}\left|\left(D \widetilde{s}_{j}\right)^{v}\right|_{\widetilde{J}^{\epsilon}}^{2}=0 .
$$

We will treat the case (8.33) in the end of subsection 8.5 and prove that $\widetilde{s}_{j}$ bubbles-off as $j \rightarrow \infty$. We first study the case (8.34) in the next subsection.

\subsection{Construction of solutions II; analysis of the thin parts.}

We go back to the original sequence $s_{j}$. We can regard the equation of pseudoholomorphic sections with the asymptotic conditions (8.25) as the following equivalent system of perturbed Cauchy-Riemann equations:

$$
\begin{aligned}
& \left.\bar{\partial}_{\widetilde{J}} s_{i}\right|_{C_{i}}=0 \text { for } i=1,2,3 \\
& \left.s_{1}\right|_{\theta_{3}}=\left.s_{3}\right|_{\theta_{3}},\left.s_{2}\right|_{\theta_{2}}=\left.\widetilde{s}_{1}\right|_{\theta_{2}},\left.s_{3}\right|_{\theta_{1}}=\left.s_{2}\right|_{\theta_{1}} .
\end{aligned}
$$

We will study (8.35-i) for each $i=1,2,3$ separately with the matching condition (8.36) in our mind. 
We start with (8.35-3). It follows from (8.34) and the conformal invariance of the vertical energy that we have the energy bound

$$
\frac{1}{2} \int_{\Sigma_{3}}\left|\left(D s_{j}\right)^{v}\right|_{\widetilde{J}}^{2} \leq \delta_{j} \rightarrow 0
$$

as $j \rightarrow \infty$. On $\left[r_{3, \text { rig }}, \infty\right) \times S^{1},(8.35-3)$ is equivalent to

$$
\frac{\partial u}{\partial \tau}+J_{t}^{\epsilon}\left(\frac{\partial u}{\partial t}-X_{\epsilon f}\right)=0
$$

for the section $s(\tau, t)=(\tau, t, u(\tau, t))$ where $J_{t}^{\epsilon}=\left(\phi_{\epsilon f}^{t}\right)_{*} J_{3}^{\prime}$ with $J_{3}^{\prime} \in j_{\left(\phi_{\epsilon f}^{1}, J_{0}\right)}$. We fix $0<r_{i, l e f}<r_{i, r i g}$ for the following discussion until the end of the discussion on $\Sigma_{3}$. Only at the end, we will let $r_{3, \text { rig }}$ go to zero.

We recall that $J_{t}^{\epsilon} \rightarrow J_{0}$ as $\epsilon \rightarrow 0$. The energy bound (8.37) and the $\epsilon$-regularity theorem (see Lemma 4.2) in the context of pseudo-holomorphic curves, which can be easily modified for (8.38) using the fact that $\left|X_{\epsilon f}\right| \rightarrow 0$ in the $C^{\infty}$-topology as $\epsilon \rightarrow 0$, imply the following uniform $C^{1}$-estimate. Here we would like to emphasize that we implicitly use the fact that any disc of a fixed size in the cylinder $\mathbb{R} \times S^{1}=\mathbb{R} \times(\mathbb{R} / \mathbb{Z})$ is conformally isomorphic to the standard disc $D=D^{2}(1)$. This kind of (uniform) estimate does not exist for the rescaled maps $\widetilde{s}_{j}$ as $\epsilon \rightarrow 0$, for which the conformal structures of the domain cylinders degenerate as $\epsilon \rightarrow 0$.

Lemma 8.5. Let $\delta_{j}>0$ as in (8.37). Then there exists $j_{0}$ such that for any $j \geq j_{0}$ and $u$, we have

$$
\left|D u_{j}(\tau, t)\right| \leq C\left(\delta_{j}+\epsilon_{j}\right)
$$

on $\Sigma_{3}=\mathbb{R}_{+} \times S^{1}$ with $C>0$ independent of $j$. In particular, we have

$$
\operatorname{length}\left(t \mapsto u_{j}(\tau, t)\right) \leq C\left(\delta_{j}+\epsilon_{j}\right) .
$$

for any $\tau \in[0, \infty)$.

Proof. We define

$$
v_{j}(\tau, t)=\left(\phi_{\epsilon f}^{t}\right)^{-1}\left(u_{j}(\tau, t)\right)
$$

on $\left[r_{3, \text { rig }}, \infty\right) \times[0,1]$ and extend to $[1 / 2, \infty) \times \mathbb{R}$ by the formula

$$
\phi_{\epsilon_{j} f}^{1}\left(v_{j}(\tau, t+1)\right)=v_{j}(\tau, t)
$$

as before. Then it follows that $v_{j}$ satisfy

$$
\frac{\partial v}{\partial \tau}+J_{3}^{\prime} \frac{\partial v}{\partial t}=0
$$

and the energy bound

$$
\frac{1}{2} \int_{\left[r_{3, r i g}, \infty\right) \times[0,1]}\left|D v_{j}\right|_{J_{3, t}^{\prime}}^{2} d t \leq \delta_{j} .
$$

from (8.37). Furthermore by the remark right above Lemma 2.14, we obtain

$$
\frac{1}{2} \int_{\left[r_{3, r i g}, \infty\right) \times[-1 / 2,3 / 2]}\left|D v_{j}\right|_{J_{3, t}^{\prime}}^{2} d t \leq 2 \delta_{j} .
$$


The above equation is not precisely the equation of the type dealt in the $\epsilon$-regularity theorem, Lemma 4.2, in that here $J_{3}^{\prime}$ is t-dependent. However an examination of the proof of [Proposition 3.3, Oh1] shows that the proof goes through for the current case where $J_{3}^{\prime}$ varies depending on the domain variable $(\tau, t)$ of $v$, and the same $\epsilon$ regularity theorem holds for equation (8.38). Once we have the $\epsilon$-regularity theorem at our disposal, we cover $\left[2 r_{3, \text { rig }}, \infty\right) \times[0,1]$ by discs of a fixed radius, say $r_{3, \text { rig }}$, whose center lie at $\left[2 r_{3, \text { rig }}, \infty\right) \times[0,1]$. Note that any such disc is contained in $\left[r_{3, \text { rig }}, \infty\right) \times[-1 / 2,3 / 2]$. We choose $j_{0}$ so large that $2 \delta_{j}<\epsilon$ for all $j \geq j_{0}$ where $\epsilon$ is the constant appearing in the $\epsilon$-regularity theorem, Lemma 4.2. Then, by applying Corollary 4.3 to each of these discs, we obtain the uniform $C^{1}$-estimate

$$
\left|D v_{j}(\tau, t)\right| \leq C \delta_{j} \rightarrow 0
$$

on $\left[2 r_{3, \text { rig }}, \infty\right) \times[0,1]$ for a uniform constant $C>0$ as $j \geq j_{0}$. Since $u_{j}(\tau, t)=$ $\phi_{\epsilon_{j} f}^{t}\left(v_{j}(\tau, t)\right)$ by the definition of $v_{j}$, we have

$$
\begin{aligned}
\frac{\partial u_{j}}{\partial \tau} & =T \phi_{\epsilon_{j} f}^{t}\left(\frac{\partial v_{j}}{\partial \tau}\right) \\
\frac{\partial u_{j}}{\partial t} & =T \phi_{\epsilon_{j} f}^{t}\left(\frac{\partial u_{j}}{\partial \tau}\right)+X_{\epsilon_{j} f}\left(u_{j}(\tau, t)\right) \\
& =T \phi_{\epsilon_{j} f}^{t}\left(\frac{\partial u_{j}}{\partial \tau}\right)+\epsilon_{j} X_{f}\left(u_{j}(\tau, t)\right) .
\end{aligned}
$$

Therefore we obtain

$$
\begin{aligned}
\left|D u_{j}\right|^{2} & =\left|\frac{\partial u_{j}}{\partial \tau}\right|^{2}+\left|\frac{\partial u_{j}}{\partial t}\right|^{2} \\
& =\left|T \phi_{\epsilon_{j} f}^{t}\left(\frac{\partial v_{j}}{\partial \tau}\right)\right|^{2}+\left|T \phi_{\epsilon_{j} f}^{t}\left(\frac{\partial v_{j}}{\partial \tau}\right)+\epsilon_{j} X_{f}\left(u_{j}(\tau, t)\right)\right|^{2} \\
& \leq 2\left|T \phi_{\epsilon_{j} f}^{t}\right|^{2}\left(\left|\frac{\partial v_{j}}{\partial \tau}\right|^{2}+\left|\frac{\partial v_{j}}{\partial \tau}\right|^{2}\right)+2 \epsilon_{j}^{2}\left|X_{f}\left(u_{j}(\tau, t)\right)\right|^{2} .
\end{aligned}
$$

The lemma immediately follows from this and (8.42) by adjusting the constant $C>0$ appropriately.

We reparameterize $u_{j}$ to define

$$
\bar{u}_{j}(\tau, t)=u_{j}\left(\frac{\tau}{\epsilon_{j}}, \frac{t}{\epsilon_{j}}\right)
$$

on $\left[2 \epsilon_{j} r_{3, \text { rig }}, \infty\right) \times \mathbb{R} / \epsilon_{j} \mathbb{Z}$. Then $\bar{u}_{j}$ satisfies

$$
\frac{\partial \bar{u}_{j}}{\partial \tau}(\tau, t)+J_{t}^{\epsilon_{j}} \frac{\partial \bar{u}_{j}}{\partial t}(\tau, t)-\operatorname{grad}_{g_{J_{t}} \epsilon_{j}} f\left(\bar{u}_{j}\right)(\tau, t)=0 .
$$

We denote grad for $\operatorname{grad}_{g_{J_{0}}}$ from now on. We choose the 'center of mass' for each circle $t \mapsto u_{j}(\tau, t)$ which we denote by $\chi_{j}(\tau)$. More precisely, $\chi_{j}(\tau)$ is defined by the following standard lemma. (See $[\mathrm{K}]$ for its proof.) 
Lemma 8.6. Suppose a circle $z: S^{1} \rightarrow M$ has diameter less than $\delta$ with $\delta=\delta(M)$ sufficiently small and depending only on $M$. Then there exists a unique point $x_{z}$, which we call the center of mass of $z$, such that

$$
z(t)=\exp _{x_{z}} \xi(t), \quad \int_{S^{1}} \xi(t) d t=0 .
$$

Furthermore $x_{z}$ depends smoothly on $z$ but does not depend on its parametrization.

If $j_{0}$ is sufficiently large, (8.39) implies that the diameter of each of the circles $t \mapsto u_{j}(\tau, t)$ is less than $\delta$ given in Lemma 8.5 for any $j \geq j_{0}$ and $\tau \in\left[2 \epsilon_{j} r_{3, \text { rig }}, \infty\right)$. Therefore we can write $\bar{u}_{j}(\tau, t)$ as

$$
\bar{u}_{j}(\tau, t)=\exp _{\chi_{j}(\tau)}\left(\bar{\xi}_{j}(\tau, t)\right)
$$

with $\bar{\xi}_{j}(\tau, t) \in T_{\chi_{j}(\tau)} M$ for any $\tau \in\left[\epsilon_{j} r_{3, \text { rig }}, \infty\right)$ for all $j \geq j_{0}, j_{0}$ sufficiently large. Furthermore the $C^{1}$ estimate in Lemma 8.5 proves

$$
|\bar{\xi}(\tau, t)| \leq C \delta_{j} .
$$

We consider the exponential map

$$
\exp : \mathcal{U} \subset T M \rightarrow M ; \quad \exp (x, \xi):=\exp _{x}(\xi)
$$

and denote

$$
D_{1} \exp (x, \xi): T_{x} M \rightarrow T_{x} M
$$

the (covariant) partial derivative with respect to $x$ and

$$
d_{2} \exp (x, \xi): T_{x} M \rightarrow T_{x} M
$$

the usual derivative $d_{2} \exp (x, \xi):=T_{\xi} \exp _{x}: T_{x} M \rightarrow T_{x} M$. We recall the property

$$
D_{1} \exp (x, 0)=d_{2} \exp (x, 0)=i d
$$

which is easy to check. We now compute

$$
\begin{aligned}
& \frac{\partial \bar{u}}{\partial \tau}=d_{2} \exp (\chi(\tau), \bar{\xi}(\tau, t))\left(\frac{D \bar{\xi}}{\partial \tau}(\tau, t)\right)+D_{1} \exp (\chi(\tau), \bar{\xi}(\tau, t))(\dot{\chi}(\tau)) \\
& \frac{\partial \bar{u}}{\partial t}=d_{2} \exp (\chi(\tau), \bar{\xi}(\tau, t))\left(\frac{\partial \bar{\xi}}{\partial t}(\tau, t)\right) \\
& \operatorname{grad} f(\bar{u})=\operatorname{grad} f\left(\exp _{\chi(\tau)} \bar{\xi}(\tau, t)\right)
\end{aligned}
$$

Substituting these into (8.43) and multiplying $\left(d_{2} \exp \left(\chi_{j}(\tau), \bar{\xi}_{j}(\tau, t)\right)\right)^{-1}$ to the resulting equation, we get

$$
\begin{aligned}
\frac{D \bar{\xi}_{j}}{\partial \tau}(\tau, t) & +\left(d_{2} \exp \left(\chi_{j}(\tau), \bar{\xi}_{j}(\tau, t)\right)\right)^{-1}\left(D_{1} \exp \left(\chi_{j}(\tau), \bar{\xi}_{j}(\tau, t)\right)\left(\dot{\chi}_{j}(\tau)\right)\right) \\
& +\left(\exp _{\chi_{j}(\tau)}\right)^{*} J_{t}^{\epsilon}\left(\chi_{j}(\tau)\right)\left(\frac{\partial \bar{\xi}_{j}}{\partial t}\right)(\tau, t)-\left(\exp _{\chi_{j}(\tau)}\right)^{*}(\operatorname{grad} f)\left(\bar{\xi}_{j}(\tau, t)\right)=0
\end{aligned}
$$


on $T_{\chi_{j}(\tau)} M$. Using the center of mass condition in Lemma 8.6 and integrating over $t \in \mathbb{R} / \epsilon_{j} \mathbb{Z}$, we obtain

$$
\begin{gathered}
\int_{0}^{\epsilon_{j}}\left(d_{2} \exp \left(\chi_{j}(\tau), \bar{\xi}_{j}(\tau, t)\right)\right)^{-1}\left(D_{1} \exp \left(\chi_{j}(\tau), \bar{\xi}_{j}(\tau, t)\right)\left(\dot{\chi}_{j}(\tau)\right)\right) d t \\
-\int_{0}^{1}\left(\exp _{\chi_{j}(\tau)}\right)^{*}(\operatorname{grad} f)\left(\bar{\xi}_{j}(\tau, t)\right) d t=0 .
\end{gathered}
$$

Here we used the identities

$$
\begin{aligned}
& \int_{S^{1}} \frac{\partial \bar{\xi}_{j}}{\partial t}(\tau, t) d t=0 \\
& \int_{S^{1}} \bar{\xi}_{j}(\tau, t) d t \equiv 0 \equiv \int_{S^{1}} \frac{D \bar{\xi}_{j}}{\partial \tau}(\tau, t) d t
\end{aligned}
$$

where the second identity of (8.46) is a consequence of the first. On the other hand, it follows that there exists sufficiently small $\delta_{j}>0$ such that

$$
\begin{aligned}
\left(1-C \delta_{j}\right)|\dot{\chi}(\tau)| & \left.\leq \mid d_{2} \exp \left(\chi_{j}(\tau), \bar{\xi}(\tau, t)\right)\right)^{-1}\left(D_{1} \exp (\chi(\tau), \bar{\xi}(\tau, t))(\dot{\chi}(\tau))\right) \mid \\
& \leq\left(1+C \delta_{j}\right)|\dot{\chi}(\tau)|
\end{aligned}
$$

and

$$
\left|\left(\exp _{\chi(\tau)}\right)^{*}(\operatorname{grad} f)(\xi(\tau, t))\right| \leq\left(1+C \delta_{j}\right)|\operatorname{grad} f(\xi(\tau, t))| \leq\left(1+C \delta_{j}\right)|| \operatorname{grad} f \|_{C^{0}}
$$

hold. These follow from (8.44) and the following standard estimates on the exponential map

$$
\begin{array}{r}
\left|d_{2} \exp (x, \xi)(u)\right| \leq C|\xi||u| \\
\left|D_{1} \exp (x, \xi)(u)\right| \leq C|\xi||u|
\end{array}
$$

for $\xi, u \in T_{x} M$ where $C$ is independent of $\xi$, as long as $|\xi|$ is sufficiently small, say smaller than injectivity radius of the metric on $M$. (see $[\mathrm{K}]$.)

Combining (8.44), (8.47) and (8.48), we have obtained

$$
\left|\dot{\chi}_{j}(\tau)\right| \leq C\|\operatorname{grad} f\|_{C^{0}}
$$

Therefore $\chi_{j}$ is equi-continuous on any given fixed interval $[\eta, R] \subset(0, \infty)$ for $\eta$ taken arbitrarily small and $R$ taken arbitrarily large. Here we recall that $2 \epsilon_{j} r_{3, \text { rig }} \rightarrow$ 0 as $j \rightarrow \infty$. Therefore there exists a subsequence of $\chi_{j}:[\eta, R] \rightarrow M$ uniformly convergent to some $\chi_{\infty}:[\eta, R] \rightarrow M$. Furthermore it easily follows from (8.48) and smoothness of the exponential map, we also have the estimates

$$
\begin{aligned}
\left.\mid d_{2} \exp \left(\chi_{j}(\tau), \bar{\xi}_{j}(\tau, t)\right)\right)^{-1} & \left(D_{1} \exp \left(\chi_{j}(\tau), \bar{\xi}_{j}(\tau, t)\right)\left(\dot{\chi}_{j}(\tau)\right)-\dot{\chi}_{j}(\tau) \mid\right. \\
& \leq C\left|\bar{\xi}_{j}(\tau, t)\right|\left|\dot{\chi}_{j}(\tau)\right|
\end{aligned}
$$

and

$$
\left|\exp _{\chi_{j}(\tau)}^{*}(\operatorname{grad} f)\left(\bar{\xi}_{j}(\tau, t)\right)-\operatorname{grad} f\left(\chi_{j}(\tau)\right)\right| \leq C\left|\bar{\xi}_{j}(\tau, t)\right|
$$


where the constant $C$ depends only on $M$. Since $\max \left|\bar{\xi}_{j}\right|_{C^{0}} \leq C \delta_{j} \rightarrow 0$ uniformly, the equation (8.44) converges uniformly to

$$
\dot{\chi}_{\infty}-\operatorname{grad} f\left(\chi_{\infty}\right)=0 .
$$

Therefore $\chi_{\infty}$ is a gradient trajectory of $f$ defined on $[\eta, R] \subset(0, \infty)$. Recalling the $C^{1}$-estimate in Lemma 8.5 and

$$
\bar{u}_{j}(\tau, t)=\exp _{\chi_{j}(\tau)}(\bar{\xi}(\tau, t))
$$

we have proven that $\left.\bar{u}_{j}\right|_{[\eta, R] \times\left(\mathbb{R} / \epsilon_{j} \mathbb{Z}\right)}$ uniformly converges to $\chi_{\infty}$. By a boot-strap argument by differentiating (8.44), this convergence can be turned into a $C^{\infty}$ convergence.

Therefore letting $\eta \rightarrow 0$ and $R \rightarrow \infty$, by a standard argument of local convergence as in [Fl2], we prove that the sequence $u_{j}$ converges to a connected finite union of gradient trajectories of $-f$

$$
\chi_{0} \# \chi_{1} \# \cdots \# \chi_{N}
$$

for some $N \in \mathbb{Z}_{+}$where $\chi_{0}$ is defined on $[0, \infty)$ and all other $\chi_{i}$ 's are on $\mathbb{R}$, and $\chi_{N}$ satisfies

$$
\chi_{N}(\infty)=q
$$

However since $\mu_{-\epsilon f}^{\text {Morse }}(q)=2 n$, i.e, $q$ is a (local) maximum of $-f, \chi_{N}$ must be constant which in turn implies that all $\chi_{k}$ must be the constant map $q$ for all $k=1, \cdots, N$. Going back to the original variable $(\tau, t) \in\left[2 r_{3, \text { rig }}, \infty\right) \times \mathbb{R} / \mathbb{Z}$ and recalling

$$
u_{j}(\tau, t)=\bar{u}_{j}\left(\epsilon_{j} \tau, \epsilon_{j} t\right)
$$

we have proven that $\left.u_{j}\right|_{\left[2 r_{3, \text { rig }}, \infty\right) \times S^{1}}$ converges to the constant map $q$ in the (fine) $C^{\infty}$-topology.

Finally, we repeat the above whole process for a sequence of $r_{3, \text { rig }} \rightarrow 0$ and conclude that $\left.u_{j}\right|_{\Sigma_{3} \times S^{1}}$ converges to the constant map $q$ in the (fine) $C^{\infty}$-topology, by the standard argument of taking a diagonal subsequence. From now on, we assume this.

\subsection{Construction of solutions III: wrap-up.}

We have established that $\left.u_{j}\right|_{\Sigma_{3}^{\epsilon} \times S^{1}}$ converges to the constant map $q$ in the (fine) $C^{\infty}$-topology under the condition (8.34). In particular $\left.s\right|_{D\left(\delta\left(\epsilon_{j}\right) \backslash\{(0,0)\}\right.}$ converges to the constant point $((0,0), q) \in \Sigma \times M=P$ and its restriction $\left.s\right|_{\partial D\left(\delta\left(\epsilon_{j}\right)\right.}$. Now we consider the restriction

$$
s_{j}:\left.\Sigma \backslash D\left(\delta\left(\epsilon_{j}\right)\right) \rightarrow P\right|_{\Sigma \backslash D\left(\delta\left(\epsilon_{j}\right)\right)}
$$

of the section $s_{j}: \Sigma \rightarrow P$. Since $\left.s_{j}\right|_{\partial D\left(\delta\left(\epsilon_{j}\right)\right)}$ converges to $((0,0), q)$ in the $C^{\infty}$ topology, we can smoothly extend $\left.s_{j}\right|_{\partial D\left(\delta\left(\epsilon_{j}\right)\right)}$ into the interior of $D\left(\delta\left(\epsilon_{j}\right)\right)$ : Apply Lemma 8.6 and write $\Phi\left(s_{j}(t)\right)=\left(t, u_{j}(t)\right)$ on $t \in \partial D\left(\delta\left(\epsilon_{j}\right)\right)$ as

$$
u_{j}(t)=\exp _{q} \xi_{j}(t), \quad \int_{S^{1}} \xi_{j}(t) d t=0
$$


and then we extend this to a smooth map $u_{j}^{\prime}: D\left(\delta\left(\epsilon_{j}\right)\right) \rightarrow M$ this by the formula

$$
u_{j}^{\prime}(z)=\exp _{q}\left(r \xi_{j}(\theta)\right)
$$

$(r, \theta)$ is the polar coordinates of $z \in D\left(\delta\left(\epsilon_{j}\right)\right)$, i.e., $z=r+\sqrt{-1} \theta$. Now we consider a modified sequence $s_{j}^{\prime}: \Sigma \rightarrow P$ of the sections $\widetilde{s}_{j}$ defined by

$$
s_{j}^{\prime}(z)= \begin{cases}s(z) & z \in \Sigma \backslash D\left(\delta\left(\epsilon_{j}\right)\right) \\ u_{j}^{\prime}(z) & z \in D\left(\delta\left(\epsilon_{j}\right)\right) .\end{cases}
$$

By flattening the boundary of $u^{\prime}$ reparameterizing the $r$ variable, we can make $s_{j}^{\prime}$ a smooth section for each $j$. It follows that $s_{j}^{\prime}$ is $\widetilde{J}$-holomorphic on $\Sigma \backslash D\left(\delta\left(\epsilon_{j}\right)\right)$ and $\left|D s_{j}^{\prime}\right|_{D\left(\delta\left(\epsilon_{j}\right)\right)} \rightarrow 0$ as $j \rightarrow \infty$ by construction.

As we mentioned before the lower bound (8.27) implies, by the definition of the Novikov chains, that there are only finitely many elements $[z, w] \in \alpha_{H}$ and $\left[\widetilde{z}^{\prime}, \widetilde{w}^{\prime}\right] \in \beta_{\widetilde{H}}$ above the lower bound. Therefore we can now apply Gromov-Floer type compactness arguments on $\Sigma$ as $j \rightarrow \infty$ to the approximately $\widetilde{J}$-holomorphic sections $s_{j}^{\prime}$.

We note that by the choice of minimal area metric representation of $\Sigma$ given in (8.29), the union $\Sigma_{1}^{\epsilon} \cup \Sigma_{2}^{\epsilon}$ converges to $\Sigma \backslash\{(0,0)\}$ as $\epsilon \rightarrow 0$ in the metric sense.

When $s_{j}^{\prime}$ bubbles-off, it follows from the $(H, J)$-compatibility of $\widetilde{J}$ via the maximum principle that the bubble $u$ must be contained in a fiber. Here we recall the relation $J_{t}=\left(\phi_{J}^{t}\right)_{*} J_{t}^{\prime}$. If we define

$$
v(z):=\left(\phi_{H}^{t}\right)^{-1}(u(z)),
$$

$v$ is a $J_{t}^{\prime}$-holomorphic sphere satisfying

$$
\omega(v)=\omega(u) \geq A_{S}\left(\phi, J_{0} ; J^{\prime}\right)
$$

where the inequality comes from the definition (1.10) of $A_{S}\left(\phi, J_{0} ; J^{\prime}\right)$. Therefore we have

$$
\limsup _{j \rightarrow \infty} \frac{1}{2} \int\left|\left(D s_{j}\right)^{v}\right|_{\widetilde{J}^{\epsilon}}^{2}=\limsup _{j \rightarrow \infty} \frac{1}{2} \int\left|\left(D \widetilde{s}_{j}\right)^{v}\right|_{\widetilde{J}^{\epsilon}}^{2} \geq A_{S}\left(\phi, J_{0} ; J^{\prime}\right)
$$

and are in the second alternative of Theorem 5.4.

Therefore, by choosing a subsequence if necessary, it remains to consider the case where there exists a constant $C>0$ such that

$$
\left|D s_{j}^{\prime}\right|_{\widetilde{J}} \leq C .
$$

Here we point out that by the definition of the data $\left(P, \widetilde{J}^{\epsilon}, H^{\epsilon}\right)$ and by the choice of minimal area metrics $g_{\epsilon}$ as in $(8.1)$, the bundle data $\left(P, \widetilde{J}^{\epsilon}\right)$ on $\mathbb{R} \times S^{1} \backslash\{(0,0)\}$ smoothly converge to $(P, \widetilde{J})$ on compact subsets of $\mathbb{R} \times S^{1} \backslash\{(0,0)\}$. Note that the latter datum is smooth.

Using the $C^{1}$-bound (8.51) and this smooth convergence of the data $\left(H^{\epsilon}, \widetilde{J}^{\epsilon}\right)$ to $(H, \widetilde{J})$ we can bootstrap to extract a broken-trajectory limit $s_{\infty}$ on $\Sigma=\Sigma^{-} \cup \Sigma^{+}$ such that

$$
s_{\infty}=\left(s_{1}^{-} \# s_{2}^{-} \# \cdots \# s_{N}^{-}\right) \#\left(s_{1}^{+} \# \cdots \# s_{L}^{+}\right)
$$


where $s_{i}^{-}$for $1 \leq i \leq N-1$ and $s_{j}^{+}$for $2 \leq i \leq L$ are maps from $\mathbb{R} \times[0,1]$ to $M$ but $s_{N}^{-}$a map from $\Sigma^{-}=(-\infty, 0] \times S^{1}$ and $s_{1}^{+}$from $\Sigma^{+}=[0, \infty) \times S^{1}$. Now the uniform $C^{1}$-bound (8.51) of $\widetilde{s}_{j}$ implies that $s_{N}^{-} \# s_{1}^{+}$becomes indeed smooth even across $\tau=0$ by the elliptic regularity. Hence we have produced a smooth broken-trajectory solution of the equation (8.52).

If we write $\left.s\right|_{\Sigma_{i}}$ in terms of the mapping cylinder of $\phi$, all of $v_{i}^{ \pm}$satisfy

$$
\left\{\begin{array}{l}
\frac{\partial v}{\partial \tau}+J_{t}^{\prime} \frac{\partial v}{\partial t}=0 \\
\phi(v(\tau, 1))=v(\tau, 0), \quad \int\left|\frac{\partial v}{\partial \tau}\right|_{J_{t}^{\prime}}^{2}<\infty
\end{array}\right.
$$

In particular, the join $s_{N}^{-} \# s_{1}^{+}$also satisfies $(8.52)$ on $\mathbb{R} \times[0,1]$.

Now we consider $u_{\infty}: \mathbb{R} \times S^{1} \rightarrow M$ defined by

$$
u_{\infty}(\tau, t)=\left(\phi_{H}^{t}\right)\left(v_{\infty}(\tau, t)\right) .
$$

The topological hypothesis

$$
\left[u \cup\left(\bigcup_{i=1}^{3} w_{i}\right)\right]=0 \quad \text { in } \widetilde{\pi}_{2}(M)
$$

in the definition of the pants product (see Definition 8.1) gives rise to the basic topological condition (2.11), after attaching all the possible bubbles to $u_{\infty}$ that we removed in the process. Furthermore

$$
v(0,0)=u(0,0)=q \in B(\lambda)
$$

while $v( \pm \infty) \in$ Fix $\phi$ and hence $v$ cannot be constant. This gives rise to the alternative (1) of Theorem 5.4.

Finally we consider the case (8.33). We fix a sufficiently small $\delta_{0}>0$ so that (A.1) in Appendix holds. In particular, since $\widetilde{s}_{j}$ have the uniform energy bound (8.32), there exists $C>0$ such that

$$
\frac{1}{2} \int_{D^{2}\left(\delta_{0}\right) \backslash\{(0,0)\}}\left|\left(D \widetilde{s}_{j}\right)^{v}\right|_{\widetilde{J}^{\epsilon}}^{2}<C
$$

for all $j$, where $D^{2}\left(\delta_{0}\right) \backslash\{(0,0)\} \subset C \backslash\{(0,0)\}$. Under the conformal map

$$
\begin{aligned}
\left.\psi_{j}:\left[\frac{1}{2 \pi} \ln \left(\delta\left(\epsilon_{j}\right) / \delta_{0}\right)\right), \infty\right) \times S^{1} & \rightarrow D^{2}\left(\delta_{0}\right) \backslash\{(0,0)\} ; \\
\psi_{j}(\tau, t) & =\delta\left(\epsilon_{j}\right) e^{-2 \pi(\tau+i t)}
\end{aligned}
$$

$D^{2}\left(\delta\left(\epsilon_{j}\right)\right) \backslash\{(0,0)\}$ corresponds to $[0, \infty) \times S^{1}$. We denote

$$
\bar{s}_{j}=\widetilde{s}_{j} \circ \psi_{j}
$$

If we write

$$
\Phi_{\epsilon_{j}}\left(\bar{s}_{j}\right)(\tau, t)=\left(\tau, t, u_{j}(\tau, t)\right)
$$


in the trivialization $\Phi_{\epsilon}: P \rightarrow S \times M$ defined as before, the equation $\bar{\partial}_{\bar{J}_{j}} \bar{s}_{j}=0$ on $D^{2}\left(\delta\left(\epsilon_{j}\right)\right) \backslash\{(0,0)\}$ corresponds to

$$
\frac{\partial u_{j}}{\partial \tau}+J_{\epsilon_{j}, t}^{3}\left(\frac{\partial u_{j}}{\partial t}-\epsilon_{j} X_{f}\right)=0
$$

on $[0, \infty) \times S^{1}$ similarly to (8.38). Here we recall from (8.15) that $J_{\epsilon_{j}, t}^{3} \equiv J_{0}$ at the end of $[0, \infty) \times S^{1}$. Due to the asymptotic condition $\lim _{\tau \rightarrow \infty} u(\tau)=q$, a constant orbit, the solution $u_{j}$ closes up at $\infty$ and so $\widetilde{s}_{j}: D^{2}\left(\delta_{0}\right) \backslash\{(0,0)\} \rightarrow P$ extends continuously over $(0,0)$ for each $j$. Since $q$ is a critical point of a Morse function $f$, $\left|D u_{j}\right|$ decays exponentially with the decay rate $b \epsilon_{j}$ for some uniform constant $b>0$ depending only on $f$. Given this exponential decay, a straightforward calculation proves Hölder continuity of $\widetilde{s}_{j}$ at $(0,0)$ : there exist constants $C=C\left(u_{j}\right)>0$ and $\delta_{1}=\delta_{1}\left(u_{j}\right)$ such that

$$
|D \widetilde{u}|(z) \leq C|z|^{-1+b \epsilon_{j}}
$$

for all $z \neq 0$ with $|z| \leq \delta_{1}$. Here $\widetilde{u}_{j}$ is the vertical component of the local representation $\widetilde{s}_{j}(z)=\left(z, \widetilde{u}_{j}\right)$ in the trivialization $\psi_{j}^{*} \Phi_{\epsilon_{j}}$.

On the other hand, the energy concentration (8.33) implies the derivative blowup

$$
\left|\left(D \widetilde{s}_{j}\right)^{v}\left(z_{j}\right)\right| \rightarrow \infty
$$

for some sequence $z_{j} \in D^{2}\left(\delta\left(\epsilon_{j}\right)\right) \backslash\{(0,0)\}$ as $j \rightarrow \infty$.

Now we will imitate the argument from [Fl2], [Oh1] to produce a non-constant bubble. More specifically, we refer readers to the argument from p 129 - p 130 of [Oh1]. We fix $\delta_{0}>0$ as before and consider a pair of discs $D^{\prime} \subset D$ given by

$$
D=D^{2}\left(\delta_{0}\right), \quad D^{\prime}=D^{2}\left(\frac{\delta_{0}}{2}\right) .
$$

We then consider the constants

$$
\begin{gathered}
\eta_{j}:=\inf \left\{\eta>0 \mid \text { there exists } x \in D^{2}\left(\frac{\delta_{0}}{2}\right)\right. \text { such that } \\
\left.\left\|\left(D \widetilde{s}_{j}\right)^{v}\right\|_{p, B_{\eta}(x)} \geq \eta^{2 / p-1}\right\}
\end{gathered}
$$

for a fixed constant $p>2$. Here $B_{\eta}(x)$ is the closed $\eta$-ball in $D^{2}\left(\delta_{0}\right)$ with the center lying at $x$.

Now we claim that $\eta_{j} \rightarrow 0$, after choosing a subsequence if necessary. Suppose to the contrary that $\eta_{j}$ is bounded away from zero. Then we can choose $\eta_{0}$ so that

$$
0<\eta_{0}<\min \left\{\inf _{j}\left\{\eta_{j}\right\}, \frac{\delta_{0}}{2}\right\} .
$$

and so cover $D^{2}\left(\frac{\delta_{0}}{2}\right)$ by a finite number, which is independent of $j$, of balls $B_{\eta_{0}}(x) \subset$ $D^{2}\left(\delta_{0}\right)$ such that $x \in D^{2}\left(\frac{\delta_{0}}{2}\right)$ and $\left\|\left(D \widetilde{s}_{j}\right)^{v}\right\|_{p, B_{\eta_{0}}(x)} \leq \eta_{0}^{2 / p-1}$. This would then imply the $L^{p}$-bound

$$
\left\|\left(D \widetilde{s}_{j}\right)^{v}\right\|_{p, D^{2}\left(\delta_{0} / 2\right)} \leq C\left(p, \eta_{0}, \delta_{0}\right)
$$

Using the facts that $\partial_{\widetilde{J}^{\epsilon}} \widetilde{s}_{j}=0$ and $J_{3}^{\epsilon_{k}} \rightarrow j \oplus J_{0}$ on $D^{2}\left(\delta_{0}\right) \backslash\{(0,0)\}$ (see (A.1) Appendix), (8.59) implies the uniform derivative bound

$$
\left|\left(D \widetilde{s}_{j}\right)^{v}\right|_{\infty, D^{2}\left(\delta_{0} / 3\right)} \leq C
$$


which then contradicts to (8.57). This proves $\eta_{j} \rightarrow 0$ as $j \rightarrow \infty$.

Therefore there must be some $x_{j} \in D^{2}\left(\delta_{0} / 2\right)$ such that

$$
\int_{B_{\eta_{j}}\left(x_{j}\right)}\left|\left(D \widetilde{s}_{j}\right)^{v}\right|^{p} \geq \frac{1}{2} \eta_{j}^{2-p}, \quad \eta_{j} \rightarrow 0 .
$$

Without loss of any generality, we may assume that $x_{j} \rightarrow x_{0} \in D^{2}\left(\delta_{0} / 2\right)$. Obviously we have

$$
r_{j}:=\eta_{j}^{-1} \operatorname{dist}\left(x_{j}, \partial D^{2}\left(\delta_{0}\right)\right) \rightarrow \infty .
$$

Then for every $R>0$ and $j$ large enough we have rescaled maps

$$
s_{j}^{\prime}: \mathbb{C} \supset B_{R}(0) \rightarrow P ; s_{j}^{\prime}(w)=\widetilde{s}_{j}\left(\eta_{j}\left(w-x_{j}\right)\right)
$$

satisfying

$$
\begin{aligned}
\left\|\left(D s_{j}^{\prime}\right)^{v}\right\|_{p, B_{1}(0)} & \geq \frac{1}{2} \\
\left\|\left(D s_{j}^{\prime}\right)^{v}\right\|_{p, B_{1}(x)} & \leq 1 \quad \text { for all } x \in B_{R-1}(0) .
\end{aligned}
$$

Furthermore $s_{j}^{\prime}$ satisfies the equation $\bar{\partial}_{J_{j}^{\prime}} s_{j}^{\prime}=0$ on $B(R) \backslash\{0\}$ where $J_{j}^{\prime}$ is the pull-back family of $\widetilde{J}^{\epsilon_{j}}$ under the above rescaling map

$$
w \in B_{R}(0) \rightarrow \eta_{j}\left(w-x_{j}\right) \in B_{\eta_{j}}\left(x_{j}\right) \subset D^{2}\left(\delta_{0}\right) .
$$

In particular $J_{k}^{\prime} \rightarrow j \oplus J_{0}$ on $B_{R}(0) \times M$ in the limit as $k \rightarrow \infty$. Now letting $R \rightarrow \infty$ and taking a diagonal subsequence, we have obtained a non-constant $J_{0^{-}}$ holomorphic map $v_{\infty}^{\prime}: \mathbb{C} \backslash\{0\} \rightarrow M$ with energy less than $C$. Applying the removal singularity theorem, we then obtain a nonconstant $J_{0}$-holomorphic bubble.

Combining all the above, we have finally finished the proof of Theorem 5.4.

Remark 8.7. Here we have developed our adiabatic convergence scheme only up to the level that enables us to prove that the adiabatic sequence converges to a gradient trajectory of the Morse function $f$, after renomalization, when there occurs no non-constant bubble. We hope to develop a full-scale convergence scheme of the adiabatic sequence considered in this paper elsewhere in the future.

\section{$\S 9$. Local Floer complex and its homology}

In this section, we will prove Theorem 7.1. We first note that proving the inequality $A(\phi ; 1) \geq \operatorname{osc}\left(S_{\phi}\right)$ requires to prove an existence of solution $v$ of (1.12) satisfying

$$
E_{J^{\prime}}(v) \geq \operatorname{osc}\left(S_{\phi}\right)
$$

for some $J_{0}$ and for some $J^{\prime} \in j_{\left(\phi ; J_{0}\right)}$. For this purpose, we will exploit some distinguished features of the Floer boundary operator and the pants product, when the relevant Hamiltonians $H=H^{\phi}$ as defined in (7.1), (7.2) and Theorem 7.1 are $C^{1}$-small. This distinguished feature was previously exploited by the present author in the context of Lagrangian intersection Floer theory [Oh2], which we call the thick and thin decomposition of the Floer moduli space.

We first recall the following standard invariant of $(M, \omega)$

$$
\begin{aligned}
A\left(\omega ; J_{0}\right) & =\inf \left\{\omega([u]) \mid u \text { is non-constant } J_{0} \text {-holomorphic }\right\} \\
A(\omega) & =\sup _{J_{0}} A\left(\omega ; J_{0}\right) .
\end{aligned}
$$


9.1. The thin part of the Floer boundary operator.

The following proposition will be proved in section 10 .

Proposition 9.1. Let $J_{0}$ be an almost complex structure on $M$ and $H$ have no non-constant one-periodic orbit. Let $\alpha_{i}, i=1,2$ be any two positive constants satisfying

$$
0<\alpha_{i}<A\left(\omega ; J_{0}\right), \quad \alpha_{1}+\alpha_{2}<A\left(\omega ; J_{0}\right)
$$

and any $\alpha_{3}>0$ be given. Let $J_{t}^{\prime}=\left(\phi_{H}^{t}\right)^{*} J_{0}$ as before. Let $u$ be any cusp-solution of (5.9). Then there exists a constant $\delta=\delta\left(J_{0}, \alpha\right)>0$ such that if $\|H\|<\delta$, the following alternative holds:

(1) either $u$ is 'very thin'

$$
\int\left|\frac{\partial u}{\partial \tau}\right|_{J_{0}}^{2}=\int\left|\frac{\partial v}{\partial \tau}\right|_{J_{t}^{\prime}}^{2}=\int v^{*} \omega<\alpha_{1},
$$

(2) or it is 'thick'

$$
\int\left|\frac{\partial u}{\partial \tau}\right|_{J_{0}}^{2}=\int\left|\frac{\partial v}{\partial \tau}\right|_{J_{t}^{\prime}}^{2}>A\left(\omega ; J_{0}\right)-\alpha_{2} .
$$

In addition, by choosing $\delta>0$ smaller if necessary, we can achieve

$$
\sup _{\tau \in \mathbb{R}} \operatorname{diam}(t \mapsto u(t, \tau))<\alpha_{3}
$$

for all thin solutions, which in turn implies

$$
\int\left|\frac{\partial u}{\partial \tau}\right|_{J_{0}}^{2} \leq\|H\|=\int_{0}^{1}\left(\max _{x} H_{t}-\min _{x} H_{t}\right) d t
$$

Note that any $C^{2}$-small Hamiltonian or any $C^{1}$-small autonomous Hamiltonian functions satisfy the hypotheses of this proposition. In the following discussion, we will assume one of these two conditions.

Proposition 9.1 enables us to decompose the Floer boundary operator, with respect to the Floer regular pair $(H, J)$,

$$
\partial=\partial_{0}+\partial^{\prime}
$$

where $\partial_{0}$ is the contribution from those satisfying (9.1) and $\partial^{\prime}$ the one from those satisfying (9.2). We fix

$$
\alpha_{2}<\frac{1}{4} A\left(\omega ; J_{0}\right) .
$$

As in [Oh2], by comparing the areas of the terms in the identity

$$
\partial \partial=0
$$

it follows that $\partial_{0}$ satisfies the identity

$$
\partial_{0} \partial_{0}=0
$$


We call the complex $\left(C F(H), \partial_{0}\right)$ with $\partial_{0}=\partial_{\left(J_{0}, H\right), 0}$ the little Floer complex of the pair $\left(J_{0}, H\right)$ and the corresponding homology, denoted by

$$
H F^{l i t t l e}(H):=H_{*}\left(C F(H), \partial_{0}\right)
$$

the little Floer homology of $\left(H, J_{0}\right)$ or simply of $H$.

A similar decomposition result also holds for the continuation equation (2.12) as long as the homotopy $\mathcal{H}=\left\{H^{s}\right\}_{0 \leq s \leq 1}$ is $C^{1}$-small. This gives rise to a decomposition of the chain map

$$
h_{\mathcal{H}}=h_{\mathcal{H}, 0}+h_{\mathcal{H}}^{\prime} .
$$

Again the area comparison of the thick and thin parts of the chain map identity $\partial_{H^{1}} \circ h_{\mathcal{H}}=h_{\mathcal{H}} \circ \partial_{H^{0}}$, we have

$$
\partial_{H^{1}, 0} \circ h_{\mathcal{H}, 0}=h_{\mathcal{H}, 0} \circ \partial_{H^{0}, 0} .
$$

Next we recall that if $\|H\|_{C^{1}}$ is sufficiently small, all the one-periodic orbits of $H$ are constant orbits corresponding to the fixed point set of the time-one map $\phi_{H}^{1}$. They carry the obvious constant bounding discs. This provides a distinguished subset

$$
\operatorname{Crit}^{f i x} \mathcal{A}_{H}:=\left\{[p, \widehat{p}] \mid p \in \operatorname{Fix} \phi_{H}^{1}\right\} \subset \operatorname{Crit} \mathcal{A}_{H}
$$

of Crit $\mathcal{A}_{H}$. We consider the $\mathbb{Q}$-subspace

$$
C F^{f i x}(H):=\operatorname{span}_{\mathbb{Q}}\left\{\operatorname{Crit}^{\text {fix }} \mathcal{A}_{H}\right\} \subset C F(H) .
$$

It follows from Proposition 9.1 that the subspace $C F^{f i x}(H)$ is preserved under the action of $\partial_{0}$ and hence

$$
\left(C F^{f i x}(H), \partial_{0}\right)
$$

forms a sub-complex of the little Floer complex $\left(C F(H), \partial_{0}\right)$ and satisfies

$$
\left(C F(H), \partial_{0}\right)=\left(C F^{f i x}(H), \partial_{0}\right) \otimes_{\mathbb{Q}} \Lambda_{\omega}
$$

as a chain complex. We call $\left(C F^{f i x}(H), \partial_{0}\right)$ the local Floer complex of $\left(H, J_{0}\right)$ or simply of $H$, and its associate homology the local Floer homology which we denote by $H F^{l o c}(H ; \mathbb{Q})$. By a similar discussion to the above, under the $C^{1}$-small homotopy $\mathcal{H}$ between two $H$ 's, $h_{\mathcal{H}, 0}$ induces a chain isomorphism between the two local Floer complexes.

We summarize the above discussions into the following theorem.

Theorem 9.2. Let $\delta>0$ be a sufficiently small constant and $H_{i}$ for $i=0,1$ satisfy $\left\|H_{i}\right\|<\delta$. Consider the linear homotopy

$$
\mathcal{H}: s \mapsto(1-s) H^{0}+s H^{1}
$$

and let $h_{\mathcal{H}}=h_{\mathcal{H}, 0}+h_{\mathcal{H}}^{\prime}$ be the above decomposition. Then both maps

$$
\begin{aligned}
& h_{\mathcal{H}, 0}:\left(C F\left(H^{0}\right), \partial_{0}\right) \rightarrow\left(C F\left(H^{1}\right), \partial_{0}\right) \\
& h_{\mathcal{H}, 0}:\left(C F^{f i x}\left(H^{0}\right), \partial_{0}\right) \rightarrow\left(C F^{f i x}\left(H^{1}\right), \partial_{0}\right)
\end{aligned}
$$


are chain isomorphisms and so induce isomorphisms in their homologies

$$
\begin{aligned}
& h_{\mathcal{H}, 0}: H F^{\text {little }}\left(H^{0}\right) \rightarrow H F^{\text {little }}\left(H^{1}\right), \\
& h_{\mathcal{H}, 0}: H F^{l o c}\left(H^{0} ; \mathbb{Q}\right) \rightarrow H F^{l o c}\left(H^{1} ; \mathbb{Q}\right)
\end{aligned}
$$

by choosing $\delta>0$ smaller, if necessary. In particular, we have the canonical isomorphisms

$$
\begin{aligned}
H F^{l o c}(H ; \mathbb{Q}) & \cong H_{*}(M ; \mathbb{Q}) \\
H F^{l i t t l e}(H) & \cong H F^{l o c}(H ; \mathbb{Q}) \otimes_{\mathbb{Q}} \Lambda_{\omega} \cong H(M ; \mathbb{Q}) \otimes_{\mathbb{Q}} \Lambda_{\omega} .
\end{aligned}
$$

We refer to $[\mathrm{Ch}]$ or $[\mathrm{Oh} 2,5]$ for some similar results and for more details of the proof of the theorem.

9.2. The thin part of the pants product.

Next we consider the pants product. We will be particularly interested in the case

$$
H_{1}=H^{\phi}, H_{2}=\widetilde{H}^{\phi}, H_{3}=0
$$

which we regard as the limit of the case (8.11).

In this case, again the relevant moduli space is decomposed into the thin part and the thick part, but this time we need to incorporate the setting of the Hamiltonian fibration. A similar decomposition exists for arbitrary numbers of ends, of course.

To motivate our discussion, we recall the quantum product of $a, b \in H^{*}(M)$ can be written as

$$
a \circ b=a \cap b+\sum_{A \neq 0}(a, b)_{A} q^{-A}
$$

where $(a, b)_{A} \in H^{*}(M)$ is the cohomology class defined by

$$
(a, b)_{A}=P D\left(\left[\mathcal{M}_{3}(J ; A) \times_{\left(e v_{1}, e v_{2}\right)}\left(Q_{a} \times Q_{b}\right), e v_{0}\right]\right) .
$$

Here $\mathcal{M}_{3}(J ; A)$ is the set of stable maps with three marked points $\left(x_{1}, x_{2}, x_{3}\right) \subset S^{2}$ and in class $A \in H_{2}(M)$. We denote by $e v_{i}: \mathcal{M}_{3}(J ; A) \rightarrow M$ for $i=0,1,2$ the evaluation maps. Then

$$
\mathcal{M}_{3}(J ; A) \times_{\left(e v_{1}, e v_{2}\right)}\left(Q_{a} \times Q_{b}\right)
$$

is the fiber product of $\mathcal{M}_{3}(J ; A)$ and $Q_{a}, Q_{b}$ via the evaluation maps $\left(e v_{1}, e v_{2}\right)$. $\left[\mathcal{M}_{3}^{A} \times_{\left(e v_{1}, e v_{2}\right)}\left(Q_{a} \times Q_{b}\right), e v_{0}\right]$ is the homology class of the fiber product as a chain via the map

$$
e v_{0}: \mathcal{M}_{3}(J ; A) \times_{\left(e v_{1}, e v_{2}\right)}\left(Q_{a} \times Q_{b}\right) \rightarrow M .
$$

Geometrically it is provided by the image (by $e v_{0}$ ) of the holomorphic spheres intersecting the cycles $Q_{a}$ and $Q_{b}$ at the first and second marked points. The term corresponding to $A=0$ provides the classical cup produce $a \cup b$.

We recall the pants product $\gamma * \delta$ of the Novikov cycles $\gamma$ and $\delta$ of $H_{1}$ and $H_{2}$ is defined by replacing the above $\mathcal{M}_{3}(J ; A)$ by the moduli space

$$
\mathcal{M}(H, \widetilde{J} ; \widehat{z})
$$


for $\widehat{z}=\left(\widehat{z}_{1}, \widehat{z}_{2}, \widehat{z}_{3}\right)$ with $\widehat{z}_{i}=\left[z_{i}, w_{i}\right]$. We note that when all the one-periodic orbits of $H_{1}, H_{2}, H_{3}$ are constant, any periodic orbit $z=p$ has the canonical lifting $[p, \widehat{p}]$ in $\widetilde{\Omega}_{0}(M)$. Therefore we can write the generator $[z, w]$ as

$$
p \otimes q^{A}:=[p, \widehat{p} \# A] .
$$

We fix a trivialization $P=\Sigma \times M$ and write $u=p r_{2} \circ s$.

For each given $p=\left(p_{1}, p_{2}, p_{3}\right)$ with $p_{i}$ (constant) periodic orbits of $H_{i}$ respectively, we denote by $\mathcal{M}_{3}(H, \widetilde{J} ; p)$ the set of all $\widetilde{J}$-holomorphic sections $s$ over $\Sigma$ with the obvious asymptotic conditions

$$
u\left(x_{1}\right)=p_{1}, u\left(x_{2}\right)=p_{2}, u\left(x_{3}\right)=p_{3}
$$

where $u$ is the fiber component of $s$ under the trivialization $\Phi: P \rightarrow \Sigma \times M$ and the $x_{i}$ 's are the given punctures in $\Sigma$ as described in section 8.1. If we denote by $\mathcal{M}_{3}(H, \widetilde{J})$ the set of all finite energy $\widetilde{J}$-holomorphic sections, then it has the natural decomposition

$$
\mathcal{M}_{3}(H, \widetilde{J})=\cup_{p} \mathcal{M}_{3}(H, \widetilde{J} ; \widehat{p}) .
$$

Furthermore, because of the asymptotic condition (9.7) for any element $s \in \mathcal{M}_{3}(H, \widetilde{J})$, $u=p r_{M} \circ s$ naturally compactifies and so defines a homotopy class $[u] \in \pi_{2}(M)$. In this way, $\mathcal{M}_{3}(H, \widetilde{J} ; \widehat{p})$ is further decomposed into

$$
\mathcal{M}_{3}(H, \widetilde{J} ; \widehat{p})=\cup_{A \in H_{2}(M)} \mathcal{M}_{3}^{A}(H, \widetilde{J} ; \widehat{p})
$$

where $\mathcal{M}_{3}^{A}(H, \widetilde{J} ; \widehat{p})$ is the subset of $\mathcal{M}_{3}(H, \widetilde{J} ; \widehat{p})$ in class $A$. We denote

$$
\mathcal{M}_{3}^{\prime}(H, \widetilde{J} ; \widehat{p})=\cup_{A \neq 0} \mathcal{M}_{3}^{A}(H, \widetilde{J} ; \widehat{p})
$$

and then have

$$
\mathcal{M}_{3}(H, \widetilde{J} ; \widehat{p})=\mathcal{M}_{3}^{0}(H, \widetilde{J} ; \widehat{p}) \coprod \mathcal{M}_{3}^{\prime}(H, \widetilde{J} ; \widehat{p}) .
$$

In the following proposition, the quantities for the triple $H=\left(H_{1}, H_{2} ; H_{3}\right)$ defined by

$$
\begin{aligned}
E^{-}(H) & :=E^{-}\left(H_{1}\right)+E^{-}\left(H_{2}\right)+E^{+}\left(H_{1} \# H_{2}\right) \\
E^{+}(H) & :=E^{+}\left(H_{1}\right)+E^{+}\left(H_{2}\right)+E^{-}\left(H_{1} \# H_{2}\right) \\
E(H) & :=E^{+}(H)+E^{-}(H)
\end{aligned}
$$

play a similar role for the pants products as (1.2) does for the boundary map. More elaborate discussion of these quantities will be carried out in [Oh10]. Related study of these kind of quantities is also given in [En1,2].

Proposition 9.3. Suppose that $H=\left(H_{1}, H_{2}, H_{3}\right)$ consisting of $H_{i}$ that have no non-constant periodic orbits and let $J$ be given. Fix any constants $\alpha_{i}, i=1,2$ with

$$
0<\alpha_{i}<A\left(\omega ; J_{0}\right), \quad \alpha_{1}+\alpha_{2}<A\left(\omega ; J_{0}\right) .
$$

Consider the Hamiltonian fibration $P \rightarrow \Sigma$ of $H$ equipped with $(H, J)$-compatible almost complex structure $\widetilde{J}$ with respect to the symplectic form

$$
\Omega_{P, \lambda}=\omega_{P}+\lambda \omega_{\Sigma}
$$


We denote by $s$ an element in $\mathcal{M}_{3}(H, \widetilde{J})$. Then there exists a constant $\delta=\delta\left(J_{0}, \alpha\right)$ such that for any $H=\left(H_{1}, H_{2}, H_{1} \# H_{2}\right)$ satisfying

$$
E(H), \quad \max _{i}\left\|H_{i}\right\|_{C^{1}}<\delta,
$$

we can find a coupling form $\omega_{P}$ a sufficiently small constant $\lambda>0$ for which the following alternative holds:

(1) all those $s$ with $\int s^{*} \omega_{P}=0$ are 'very thin'

$$
\int|D s|_{\widetilde{J}}^{2}<\alpha_{1}
$$

and its fiber class is $[u]=0$ or,

(2) all the elements $s$ with $\int s^{*} \omega_{P} \neq 0$ are 'thick' i.e.,

$$
\int|D s|_{\widetilde{J}}^{2}>A\left(\omega ; J_{0}\right)-\alpha_{2}
$$

For the trivial generators $\widehat{p}_{i}=\left[p_{i}, \widehat{p}_{i}\right], i=1,2$, the pants product is given by

$$
\widehat{p}_{1} * \widehat{p}_{2}=\widehat{p}_{1} *_{0} \widehat{p}_{2}+\sum_{A \neq 0} \widehat{p}_{1} *_{A} \widehat{p}_{2}
$$

where $\widehat{p}_{1} *_{A} \widehat{p}_{2}$ is defined as

$$
\widehat{p}_{1} *_{A} \widehat{p}_{2}=\sum_{p_{3} \in \operatorname{Per}\left(H_{3}\right)}\left(p_{1}, p_{2} ; p_{3}\right)_{A} \widehat{p}_{3} \otimes q^{A} .
$$

Here

$$
\left(p_{1}, p_{2} ; p_{3}\right)_{A}=\#\left(\mathcal{M}_{3}^{A}(H, \widetilde{J} ; \widehat{p})\right) .
$$

(9.17) induces the formula for arbitrary generators $[z, w]=[p, \widehat{p} \# A]$ in an obvious way.

The main point of Proposition 9.3 is that the thin part $\mathcal{M}_{3}^{0}(H, \widetilde{J})$ of the moduli space $\mathcal{M}_{3}(H, \widetilde{J})$ is 'far' from the thick part $\mathcal{M}_{3}^{\prime}(H, \widetilde{J})$, and isolated under the continuation of the whole moduli space $\mathcal{M}_{3}(H, \widetilde{J})$ via $C^{1}$-small deformations of Hamiltonians as long as the thick solutions $s$ described in Proposition 9.3 do not appear during the deformations. This is guaranteed if we require (9.11) as the asymptotic boundary condition, and if $H_{3}=H_{1} \# H_{2}$ and so the corresponding curvature of the fibration $P$ is 'flat'. (See section 8 and the proof of Proposition 9.3 in section 10 for more discussion relevant to this remark.)

This in particular implies that the term in the pants product induced by $\widehat{p}_{1} *_{0}$ $\widehat{p}_{2}$, which is the contribution of the thin part of the moduli space, is invariant in homology under the $C^{2}$-small continuation of $H$ and induces the classical cup product

$$
\cup: H^{k}(M) \times H^{\ell}(M) \rightarrow H^{k+\ell}(M) .
$$

In particular the product by the identity 1 induces an isomorphism $1: H^{0}(M) \rightarrow$ $H^{0}(M)$.

A more systematic discussions on the local complex, the pants product and their applications will be carried out in [Oh10]. 


\subsection{The canonical Floer fundamental cycle.}

In this subsection, we recall the construction and some distinguished properties of canonical Floer fundamental cycles from [Oh7]. We choose a Morse function $f$ such that $f$ has the unique global minimum point $x^{-}$and

$$
f\left(x^{-}\right)=0, \quad f\left(x^{-}\right)<f\left(x_{j}\right)
$$

for all other critical points $x_{j}$. Then we choose a fundamental Morse cycle

$$
\alpha=\alpha_{\epsilon f}=\left[x^{-}, \widehat{x}^{-}\right]+\sum_{j} a_{j}\left[x_{j}, \widehat{x}_{j}\right]
$$

where $x_{j} \in$ Crit $_{2 n}(-f)$.

Considering Floer's homotopy map $h_{\mathcal{L}}$ over the linear path

$$
\mathcal{L}: s \mapsto(1-s) \epsilon f+s H
$$

for sufficiently small $\epsilon>0$, we transfer the above fundamental Morse cycle $\alpha$ and define a fundamental Floer cycle of $H$ by

$$
\alpha_{H}:=h_{\mathcal{L}}(\alpha) \in C F(H) .
$$

This is what we call the canonical fundamental Floer cycle of $H$. We would like to emphasize that the linear homotopy $\mathcal{L}$ used for this fundamental Floer cycle can be made $C^{2}$-small if $H$ is so, and so $h_{\mathcal{L}}$ restricts to a well-defined chain isomorphism between the Morse complex of $-\epsilon f$ and the local Floer complex $\left(C F(H) ; \partial_{0}\right)$. We recall the following lemma from [Oh7]. We like to mention that any sufficiently $C^{1}$-small autonomous $H$ will satisfy the hypotheses in this lemma.

Lemma 9.4 [Proposition 4.2, Oh7]. Suppose that $H$ is a generic one-periodic such that $H_{t}$ has the unique nondegenerate global minimum $x^{-}$which is fixed and under-twisted for all $t \in[0,1]$. Suppose that $f: M \rightarrow \mathbb{R}$ is a Morse function such that $f$ has the unique global minimum point $x^{-}$and $f\left(x^{-}\right)=0$. Then the canonical fundamental cycle has the expression

$$
\alpha_{H}=\left[x^{-}, \widehat{x}^{-}\right]+\beta \in C F(H)
$$

for some Floer Novikov chain $\beta \in C F(H)$ with the inequality

$$
\lambda_{H}(\beta)<\lambda_{H}\left(\left[x^{-}, \widehat{x}^{-}\right]\right)=\int_{0}^{1}-H\left(t, x^{-}\right) d t .
$$

In particular its level satisfies

$$
\begin{aligned}
\lambda_{H}\left(\alpha_{H}\right) & =\lambda_{H}\left(\left[x^{-}, \widehat{x}^{-}\right]\right) \\
& =\int_{0}^{1}-H\left(t, x^{-}\right) d t=\int_{0}^{1}-\min H d t .
\end{aligned}
$$

The same construction applies to $\beta_{\widetilde{H}}$ by replacing the maximum and minimum points in the above construction and considering the homotopy

$$
s \mapsto(1-s)(\epsilon f)+s \widetilde{H} .
$$

The following lemma shows that the above canonical fundamental cycles $\alpha_{H}$ and $\beta_{\widetilde{H}}$ are tight in the sense of Definition 2.13 inside the local complex. The proof of this was given in [Proposition 4.5, Oh5] which is stated in terms of homological essentialness of the critical point $x^{ \pm}$. (See [Definition 13.2, Po1].) The two statements are however equivalent. 
Lemma 9.5 [Proposition 4.5, Oh5]. Suppose that $H$ is nondegenerate and quasi-autonomous with the unique fixed maximum point $x^{+}$and a fixed minimum point $x^{-}$. Suppose that $\|H\|_{C^{2}}<\delta$ with $\delta>0$ is sufficiently small. Then both $\alpha_{H}$ (respectively $\beta_{\widetilde{H}}$ ) are tight in the local complex $\left(C F^{\text {fix }}(H), \partial_{H, 0}\right)$ (respectively in $\left(C F^{f i x}(\widetilde{H}), \partial_{\widetilde{H}, 0}\right)$; that is, for any $\partial_{H, 0}$-cycle $\alpha$ satisfying $\alpha=\alpha_{H}+\partial_{H, 0}(\delta)$ for a chain $\delta \in C F^{f i x}(H)$, then

$$
\lambda_{H}(\alpha) \geq \lambda_{H}\left(\alpha_{H}\right)=\int_{0}^{1}-H\left(x^{-}\right) d t .
$$

\subsection{Proof of Theorem 7.1.}

In this subsection, we will use the isomorphism (9.10) of the local Floer complex to prove an existence theorem of 'thin' solutions of (1.12).

Let $J_{0}$ be a given almost complex structure and $H$ be given. Define $J^{\prime}$ to be the family defined by $J_{t}^{\prime}=\left(\phi_{H}^{t}\right)^{*} J_{0}$ as before. We first note that

$$
A_{S}\left(\phi_{H}^{1}, J_{0} ; J^{\prime}\right)=A\left(\omega ; J_{0}\right) .
$$

This is because there is a one-one correspondence

$$
w \mapsto \phi \circ w
$$

between the set of $J_{0}$-holomorphic spheres and that of $\phi^{*} J_{0}$-holomorphic spheres.

We go back to the study of $H^{\phi}$. Now for given $J_{0}$, let us choose $\phi$ sufficiently $C^{1}$-close to the identity so that the Hofer norm $\left\|H^{\phi}\right\|$ satisfies

$$
\left\|H^{\phi}\right\|<\frac{1}{2} A\left(\omega ; J_{0}\right)
$$

and so $\gamma(\phi) \leq \frac{1}{2} A\left(\omega ; J_{0}\right)$. By the definition of $H^{\phi}$ in section $7, d_{C^{1}}\left(\phi_{H^{\phi}}, i d\right)$ can be made arbitrarily small and so satisfies the hypotheses of Proposition 9.1. Then applying Proposition 9.1 we derive from (9.26) that any solution $v$ of (1.12) satisfying

$$
E_{J^{\prime}}(v)<\frac{1}{2} A\left(\omega ; J_{0}\right)
$$

must indeed have the energy which is precisely the same as

$$
E_{J^{\prime}}(v)=\left\|H^{\phi}\right\|=\operatorname{osc}\left(S_{\phi}\right) .
$$

Therefore to prove Theorem 7.1 it is enough to prove that (1.14) has a solution $v$ satisfying (9.27).

Now we run the existence scheme used in the proof of Theorem 5.4 in section 8 for the cycles $\alpha_{H^{\phi}}$ and $\beta_{\widetilde{H}^{\phi}}$ above. Without loss of any generality, we may assume that $H^{\phi}$ satisfies the hypothesis in Lemma 9.4 if we choose $\phi$ is sufficiently $C^{1}$-small.

In particular, the canonical fundamental cycle $\alpha_{H^{\phi}}$ has the form

$$
\alpha_{H^{\phi}}=\left[x^{-}, \widehat{x}^{-}\right]+\beta
$$


with $\lambda_{H^{\phi}}(\beta)<\mathcal{A}_{H^{\phi}}\left(\left[x^{-}, \widehat{x}^{-}\right]\right)$and is tight in the local Floer complex. Similarly we have

$$
\beta_{\widetilde{H}^{\phi}}=\left[x^{+}, \widehat{x}^{+}\right]+\widetilde{\beta}
$$

with $\lambda_{\widetilde{H}^{\phi}}(\widetilde{\beta})<\mathcal{A}_{\widetilde{H}^{\phi}}\left(\left[x^{+}, \widehat{x}^{+}\right]\right)$and tight in the corresponding local Floer complex. In practice, to run our existence scheme as in section 8 , we need to consider the admissible triple

$$
H_{1}=H^{\phi}, H_{2}=\widetilde{H^{\phi}} \#(\epsilon f), H_{3}=\epsilon f
$$

for a fixed Morse function $f$, and the corresponding cycles $\alpha_{H^{\phi}}$ and $\beta_{\widetilde{H^{\phi}} \#(\epsilon f)}$. It follows that by choosing $\epsilon$ sufficiently small, $\beta_{\widetilde{H^{\phi}} \#(\epsilon f)}$ is also tight in the local Floer complex and have the similar structure as in (9.29).

Since both $\alpha_{H}$ and $\beta_{\widetilde{H^{\phi}} \#(\epsilon f)}$ are tight in the local Floer complex, the pants product

$$
\alpha_{H} * \beta_{\widetilde{H^{\phi}} \#(\epsilon f)}
$$

has a nontrivial contribution coming from the thin moduli space

$$
\mathcal{M}_{3}^{0}(H, \widetilde{J} ; \widehat{p})
$$

defined as in subsection 9.2, for the asymptotic orbits $\widehat{p}$ consisting of

$$
\widehat{p}=\left(\left[x^{-}, \widehat{x}^{-}\right],\left[x^{+}, \widehat{x}^{+}\right] ;[q, \widehat{q}]\right)
$$

with $q \in \operatorname{Crit}(-\epsilon f)$. Since this is the case for all small $\epsilon>0$, we can now carry out the same proof as in section 8. The upshot of the above discussion for $H^{\phi}$ is that case (2) of Theorem 5.4 cannot occur for the above choices of $\phi$ and of the canonical Floer fundamental cycles $\alpha_{H}$ and $\beta_{\widetilde{H^{\phi}} \#(\epsilon f)}$. This is thanks to the inequality (5.18) imposed for such a solution in Theorem 5.4 : In the current case, for given $J_{0}$, we choose $\phi$ sufficiently $C^{1}$ close to the identity. Such a choice makes $H^{\phi}$ sufficiently $C^{0}$ small so that $\left\|H^{\phi}\right\|<\frac{1}{3} A\left(\omega ; J_{0}\right)$. Then (3.2), applied to $b=1$, implies that

$$
\rho\left(H^{\phi} ; 1\right)+\frac{\delta}{2}, \rho\left(\widetilde{H}^{\phi} ; 1\right)+\frac{\delta}{2}<\frac{2}{3} A\left(\omega ; J_{0}\right)
$$

if we choose $\delta<\frac{1}{3} A\left(\omega ; J_{0}\right)$. Now we choose $J^{\prime} \in j_{\left(\phi, J_{0}\right)}$ sufficiently $C^{1}$-close to the constant path $J_{0}$ which gives rise to

$$
A\left(\omega ; J^{\prime}\right):=\inf _{t \in[0,1]}\left\{A\left(\omega ; J_{t}^{\prime}\right)\right\} \geq \frac{2}{3} A\left(\omega ; J_{0}\right)
$$

by the lower semicontinuity property of the function $J^{\prime} \mapsto A\left(\omega ; J^{\prime}\right)$ : this lower semicontinuity can be easily proven by a similar argument as the proof of Proposition 4.4. This precludes the possibility (2) in Theorem 5.4.

Once this is the case, a simple examination of the existence proof of Theorem 5.4 in section 8 immediately gives rise to the following existence theorem of thin (broken-trajectory) solutions. 
Proposition 9.6. Let $J_{0}$ be any compatible almost complex structure. Assume that $\phi$ is sufficiently $C^{1}$-small and that the graph, graph $\phi$, is contained in the Darboux neighborhood $\mathcal{U}$ as in (7.2). Let $H^{\phi}:[0,1] \times M \rightarrow \mathbb{R}$ be the quasiautonomous Hamiltonian that generates the Hamiltonian path $\phi^{t}$ determined by (7.2). Let $\alpha_{H} \in C F_{n}\left(H^{\phi}\right), \beta_{\widetilde{H}^{\phi}} \in C F_{n}\left(\widetilde{H}^{\phi}\right)$ be as before. Then there must be a 'thin' broken-trajectory solution

$$
u=u_{1} \# u_{2} \# \cdots \# u_{N}
$$

of

$$
\left\{\begin{array}{l}
\frac{\partial u}{\partial \tau}+J_{t}\left(\frac{\partial u}{\partial t}-X_{H}(u)\right)=0 \\
u_{1}(-\infty)=\left[x^{-}, \widehat{x}^{-}\right] \in \alpha_{H}, u_{N}(\infty)=\left[x^{+}, \widehat{x}^{+}\right], \text {with }\left[\widetilde{x}^{+}, \widetilde{x^{+}}\right] \in \beta_{\widetilde{H}}, \\
u_{j}(0,0)=q \in B(u) \text { for some } j=1, \cdots, N
\end{array}\right.
$$

with $J_{t}^{\prime}=\left(\phi_{H}^{t}\right)^{*} J_{0}$, that satisfy

$$
\begin{aligned}
\mathcal{A}_{H}(u(-\infty)) & =\int_{0}^{1}-\min H_{t} d t=\int_{0}^{1}-H_{t}\left(x^{-}\right) d t=\max S_{\phi} \\
\mathcal{A}_{H}(u(\infty)) & =\int_{0}^{1}-\max H_{t} d t=\int_{0}^{1}-H_{t}\left(x^{+}\right) d t=\min S_{\phi} .
\end{aligned}
$$

Finally we are ready to finish the proof of Theorem 7.1.

Wrap-up of the proof of Theorem 7.1. We choose $\alpha_{i}<\frac{1}{3} A\left(\omega ; J_{0}\right)$ in Proposition 9.1. We will prove that for the choice of $J^{\prime}$ given by $J_{t}^{\prime}=\left(\phi_{H^{\phi}}^{t}\right)^{*} J_{0}$, we have

$$
A\left(\phi, J_{0} ; J^{\prime} ; 1\right) \geq \operatorname{osc}\left(S_{\phi}\right) .
$$

This will then finish the proof of Theorem 7.1.

Proposition 9.6 implies the existence of a thin broken-trajectory solution that satisfies

$$
u(-\infty)=\left[x^{-}, \widehat{x}^{-}\right], \quad u(\infty)=\left[x^{+}, \widehat{x}^{+}\right]
$$

and hence

$$
\mathcal{A}_{H}(u(-\infty))-\mathcal{A}_{H}(u(\infty))=\operatorname{osc}\left(S_{\phi}\right) .
$$

Furthermore it follows from Proposition 9.1 that we have

$$
\sup _{\tau \in \mathbb{R}} \operatorname{diam}\left(t \mapsto u_{j}(\tau, t)\right)<\alpha_{3}
$$

for all $j=1, \cdots, N$. We note that the asymptotic orbits of $u$ are constant loops and so each $u_{j}$ defines a cycle in $M$. In addition, Lemma 8.6 implies that the image of $u$ is homologous to a one dimensional path that is the join

$$
\chi_{1} \# \cdots \# \chi_{N}
$$

where $\chi_{j}: \mathbb{R} \rightarrow M$ is the 'center of mass' path of the loops

$$
t \mapsto u_{j}(\tau, t), \quad j=1, \cdots, N
$$


defined for each $\tau \in \mathbb{R}$ as in Lemma 8.6. This latter topological property holds for any thin cusp-solutions $u^{\prime}$. Now the same calculation as the one carried out in the proof of Lemma 4.6 gives rise to

$$
E_{J}\left(u^{\prime}\right)=\int\left(u^{\prime}\right)^{*} \omega+\int_{0}^{1}\left(-H_{t}\left(x^{-}\right)+H_{t}\left(x^{+}\right)\right) d t .
$$

Since $u^{\prime}$ is homologous to a one dimensional path and so homologous to zero as a two chain, $\int\left(u^{\prime}\right)^{*} \omega=0$. Therefore we obtain

$$
E_{J}\left(u^{\prime}\right)=\int_{0}^{1}\left(-H_{t}\left(x^{-}\right)+H_{t}\left(x^{+}\right)\right) d t=\operatorname{osc}\left(S_{\phi}\right)
$$

where the second identity follows from (7.4). This and (9.27) imply

$$
\operatorname{osc}\left(S_{\phi}\right)=E_{J}\left(u^{\prime}\right)<\frac{1}{2} A\left(\omega ; J_{0}\right),
$$

for any thin broken-trajectory solution $u^{\prime}$ connecting $\left[x^{-}, \widehat{x}^{-}\right]$and $\left[x^{+}, \widehat{x}^{+}\right]$. On the other hand, for any thick solution $u^{\prime \prime}$, we have

$$
E_{J}\left(u^{\prime \prime}\right) \geq A\left(\omega ; J_{0}\right)-\alpha_{2}>\frac{2}{3} A\left(\omega ; J_{0}\right) .
$$

Combining (9.32) and (9.33), we have indeed proved

$$
A\left(\phi, J_{0} ; J^{\prime} ; 1\right)=\operatorname{osc}\left(S_{\phi}\right)
$$

for the choice $J^{\prime}=\left\{\left(\phi_{H^{\phi}}^{t}\right)^{*} J_{0}\right\}$. By the definition of $A(\phi ; 1)$, Definition 6.2, we have proved $A(\phi ; 1) \geq \operatorname{osc}\left(S_{\phi}\right)$. This finishes the proof of Theorem 7.1.

\section{$\S 10$. Thick and thin decomposition of the Floer moduli space}

In this section, we will prove Proposition 9.1 and 9.3. Similar theorem can be proven for the higher Massey product moduli spaces with arbitrary $k$ number of punctures on $\Sigma$. The cases of our main interest correspond to $k=2,3$. We start with the case of $k=2$. We recall a similar result from [Proposition 10.1, Oh2] in the context of Lagrangian intersection Floer theory for $k=2$.

Proof of Proposition 9.1. The proof is by contradiction as in $[\mathrm{Oh} 2,5]$. Suppose to the contrary that for some $0<\alpha_{1}, \alpha_{2}<A\left(\omega ; J_{0}\right)$ there exists a sequence $\delta_{j} \rightarrow 0$, $H_{j}$ with $\left\|H_{j}\right\| \leq \delta_{j}$ and $u_{j}$ satisfying (9.2) for $H_{j}$ but with the bound

$$
\alpha_{1} \leq \int\left|\frac{\partial u_{j}}{\partial \tau}\right|_{J_{0}}^{2}=\int\left|\frac{\partial v_{j}}{\partial \tau}\right|_{J_{t}^{\prime}}^{2} \leq A\left(\omega ; J_{0}\right)-\alpha_{2}
$$

Because of the energy upper bound in (10.1), we can apply Gromov's type of compactness argument. Since we assume that any one-periodic trajectory is constant, the homotopy class $[u] \in \pi_{2}(M)$ is canonically defined for any finite energy solution $u$ and in particular for $u_{j}$. A straightforward computation shows

$$
\int\left|\frac{\partial u_{j}}{\partial \tau}\right|_{J_{0}}^{2}=\int \omega\left(\frac{\partial u_{j}}{\partial \tau}, \frac{\partial u_{j}}{\partial t}\right) d \tau d t-\int_{0}^{1}\left(H\left(u_{j}(\infty)\right)-H\left(u_{j}(-\infty)\right)\right) d t
$$


and so for the finite energy solution $u_{j}$, we derive

$$
\int\left|\frac{\partial u_{j}}{\partial \tau}\right|_{J_{0}}^{2}=\omega\left(\left[u_{j}\right]\right)-\int_{0}^{1}\left(H\left(u_{j}(\infty)\right)-H\left(u_{j}(-\infty)\right) d t \leq \omega\left(\left[u_{j}\right]\right)+\|H\| .\right.
$$

Due to the energy bound (10.1) and by a standard compactness argument, we may assume that the homotopy class $\left[u_{j}\right]=A$ fixed by choosing a subsequence if necessary. If $A=0$, then (10.3) implies

$$
\int\left|\frac{\partial u_{j}}{\partial \tau}\right|_{J_{0}}^{2} \leq\|H\| \leq \delta_{j}
$$

which contradicts the lower bound in (10.1) if $j$ is sufficiently large so that $\delta_{j}<\alpha_{1}$. Now assume that $A \neq 0$. In this case, there must be some $C>0$ and $\tau_{j} \in \mathbb{R}$ with

$$
\operatorname{diam}\left(t \mapsto u_{j}\left(t, \tau_{j}\right)\right) \geq C>0
$$

where $C$ is uniform over $j$. By translating $u_{j}$ along the direction of $\tau$, we may assume that $\tau_{j}=0$. Now if bubbling occurs, we just take the bubble to produce a non-constant $J_{0}$-holomorphic sphere. If bubbling does not occur, we take a local limit around $\tau=0$ using the energy bound (10.1), to produce a map $u: \mathbb{R} \times S^{1} \rightarrow M$ satisfying

$$
\frac{\partial u}{\partial \tau}+J_{0} \frac{\partial u}{\partial t}=0
$$

Furthermore if there occurs no bubbling and so if the sequence converges uniformly to the local limit around $\tau=0$, then the local limit cannot be constant because of (10.4) and so it must be a non-constant $J_{0}$-holomorphic cylinder with the energy bound

$$
\frac{1}{2} \int|D u|_{J_{0}}^{2} \leq A\left(\omega ; J_{0}\right)-\alpha_{2}<\infty .
$$

By the removable singularity theorem, $u$ can be compactified to a $J_{0}$-holomorphic sphere that is non-constant.

Therefore whether the sequence bubbles off or not, we have

$$
\underset{j}{\limsup } \int\left|\frac{\partial u_{j}}{\partial \tau}\right|_{J_{0}}^{2} \geq A\left(\omega ; J_{0}\right)
$$

This then contradicts the upper bound of (10.1). This finishes the proof of the alternative (9.1)-(9.2). Again by arguing by contradiction, we derive (9.3) from (10.4) and (9.4) from (10.3). This finishes the proof.

Now we consider the case with $k=3$ and give the proof of Proposition 9.3. The main idea of the proof for this case is essentially the same as that of $k=2$, except that we need set-up the appropriate Hamiltonian fibration in the proof. In the proof, we are content to give the proof of Proposition 9.3 and refrain from trying to provide the optimal form of the exposition on the various computations related to the curvature and the Hofer type quantities. We will postpone an optimal form of the exposition to [Oh10].

Proof of Proposition 9.3. For given $H=\left(H_{1}, H_{2}, H_{3}\right)$ with $H_{1} \# H_{2}=H_{3}$, we choose a Hamiltonian fibration $P \rightarrow \Sigma$ with connection $\nabla$ whose monodromy at 
the ends given by the $H_{i}$ for $i=1,2,3$ as constructed in section 8. (See [Oh9] for more details.)

In the minimal metric representation as in section 8 , we have the formula of the curvature two form

$$
K(s(\tau, t)) d \tau \wedge d t
$$

of $\nabla$ where $K$ is the function

$$
K(s(\tau, t))=\sum_{i=1}^{2} \rho_{-}^{\prime}(\tau) H_{i}(t, u(\tau, t))-\rho_{+}^{\prime}(\tau)\left(H_{1} \# H_{3}\right)(t, u(\tau, t))
$$

on each $\Sigma_{i}$ where $\Phi \circ s(\tau, t)=(\tau, t, u(\tau, t))$ in the trivialization $\Phi$. (See [section 3 , Oh9] for the derivation of this formula, more specifically the formula (3.16) of [Oh9].) We note that the curvature is concentrated near the transition regions of the cut-off functions and in particular compactly supported. Furthermore we have the integral bound for the curvature integral

$$
-E^{+}(H) \leq \int K \circ s \leq E^{-}(H) .
$$

and the $L^{\infty}$-bound for $K$

$$
-E_{\infty}^{+}(H) \leq K(s(z)) \leq E_{\infty}^{-}(H)
$$

where

$$
\begin{aligned}
& E_{\infty}^{-}(H):=\max \left\{-\min _{t, x} H_{1}(t, x),-\min _{t, x} H_{2}(t, x) ; \max _{t, x} H_{3}(t, x)\right\} \\
& E_{\infty}^{+}(H):=\max \left\{\max _{t, x} H_{1}(t, x), \max _{t, x} H_{2}(t, x) ;-\min _{t, x} H_{3}(t, x)\right\} \\
& E_{\infty}(H):=E_{\infty}^{-}(H)+E_{\infty}^{+}(H) .
\end{aligned}
$$

We note that for the triple $H=\left(H_{1}, H_{2} ; H_{1} \# H_{2}\right)$ it was shown in [GLS], [En1], [Oh9] that the coupling two form $\omega_{P}$ of the connection $\nabla$ has the property that

$$
\Omega_{P, \lambda}=\omega_{P}+\lambda \omega_{\Sigma}
$$

is non-degenerate for all $\lambda \in[\delta, \infty)$, provided $E_{\infty}(H)<\delta$. Let $\widetilde{J}$ be an $(H, J)$ compatible almost complex structure which is also compatible to the symplectic form $\Omega_{P, \lambda}$. For the simplicity of notation, we denote $|\cdot|=|\cdot|_{\widetilde{J}}$. For a $\widetilde{J}$-holomorphic section, we have the identity

$$
\frac{1}{2} \int|D s|^{2}=\int s^{*}\left(\Omega_{P, \lambda}\right)=\int s^{*} \omega_{P}+\lambda
$$

We decompose $D s=(D s)^{v}+(D s)^{h}$ into vertical and horizontal parts and write

$$
|D s|^{2}=\left|(D s)^{v}\right|^{2}+\left|(D s)^{h}\right|^{2}+2\left\langle(D s)^{v},(D s)^{h}\right\rangle .
$$

Now it is straightforward to prove

$$
\left|(D s)^{h}\right|^{2} d \tau \wedge d t=2\left(K(s) d \tau \wedge d t+\lambda \omega_{\Sigma}\right)
$$


by evaluating

$$
\begin{aligned}
\sum_{i=1}^{2}\left|(D s)^{h}\left(e_{i}\right)\right|^{2} & =\sum_{i=1}^{2} \Omega_{P, \lambda}\left((D s)^{h}\left(e_{i}\right), \widetilde{J}(D s)^{h}\left(e_{i}\right)\right) \\
& =\sum_{i=1}^{2}\left(\omega_{P}+\lambda \omega_{\Sigma}\right)\left((D s)^{h}\left(e_{i}\right), \widetilde{J}(D s)^{h}\left(e_{i}\right)\right)
\end{aligned}
$$

for an orthonormal frame $\left\{e_{1}, e_{2}\right\}=\left\{\frac{\partial}{\partial \tau}, \frac{\partial}{\partial t}\right\}$ of $T \Sigma$. We refer to [Oh9] for detailed derivation of these formulae.

Combining (10.9)-(10.10), we have derived

$$
\begin{aligned}
\frac{1}{2} \int\left|(D s)^{v}\right|^{2} & =\int s^{*} \omega_{P}+\lambda-\left(\int K(s) d \tau \wedge d t+\int \lambda \omega_{\Sigma}\right) \\
& =\int s^{*} \omega_{P}-\int K(s)
\end{aligned}
$$

which is equivalent to the formula [(4.13), Oh9]. We now divide our discussion into two.

Firstly, if $\int s^{*} \omega_{P}=0$, we derive

$$
\frac{1}{2} \int\left|(D s)^{v}\right|^{2} \leq E(H)
$$

from (10.6) and (10.11). Therefore

$$
\frac{1}{2} \int\left|(D s)^{v}\right|^{2} \leq \delta
$$

Combining (10.11)-(10.13), we have established

$$
\int|D s|^{2} \leq \alpha_{1}
$$

if $\delta>0$ is chosen sufficiently small.

Secondly, if $\int s^{*} \omega_{P} \neq 0$, then $\operatorname{pr}_{\Sigma} \circ s: \Sigma \rightarrow \Sigma$ has degree one and the fiber homotopy class $[u]$ of $s$ satisfies

$$
[u]=: A \neq 0 \in \pi_{2}(M) .
$$

Furthermore noting that as $\|H\|_{C^{1}} \rightarrow 0$, the connection can be made closer and closer to the trivial connection in the trivial fibration $P=\Sigma \times M$ and the $(H, J)$ compatible $\widetilde{J}$ also converges to the product almost complex structure $j \oplus J_{0}$ and hence the image of any $\widetilde{J}$-holomorphic section cannot be completely contained in the neighborhood of one of the obvious horizontal sections

$$
\Sigma \times\{q\}
$$


for any one fixed $q \in M$. Now consider a sequence $H_{j}$ with $\left\|H_{j}\right\|_{C^{1}} \rightarrow 0$, and $\left(H_{j}, J\right)$-compatible almost complex structure $\widetilde{J}_{j}$, and let $s_{j}$ be a sequence of $\widetilde{J}_{j}$ holomorphic sections in the fixed fiber class (10.14). In other words, if we write

$$
\Phi \circ s_{j}(z)=\left(z, u_{j}(z)\right)
$$

in the trivialization $\Phi: P \rightarrow \Sigma \times M$, then we have $\left[u_{j}\right]=A \neq 0$. Since we assume $A \neq 0$, there is a constant $C>0$ such that

$$
\operatorname{diam}\left(u_{j}\right) \geq C>0
$$

for all sufficiently large $j$ after choosing a subsequence. By applying a suitable conformal transformation on the domain, either by taking a bubble if bubble occurs or by choosing a limit when bubbling does not occur, we can produce at least one non-constant $J_{0}$-holomorphic map

$$
u_{\infty}: S^{2} \rightarrow M
$$

out of the $u_{j}$ 's as in the case $k=2$ before. Furthermore we also have the energy bound

$$
\limsup _{j \rightarrow \infty} \int\left|\left(D s_{j}\right)^{v}\right|_{\widetilde{J}_{j}}^{2} \geq \int\left|D u_{\infty}\right|_{J_{0}}^{2}
$$

Therefore we have

$$
\int\left|D s_{j}\right|^{2} \geq \int\left|\left(D s_{j}\right)^{v}\right|^{2} \geq \int\left|D u_{\infty}\right|_{J_{0}}^{2} \geq A\left(\omega ; J_{0}\right)-\alpha_{2}
$$

for a sufficiently large $j$. This finishes the proof of Proposition 9.1.

Remark 10.1. In the above proof of Proposition 9.3, the readers might be wondering why we are short of stating

"By the Gromov compactness theorem, the sequence $s_{j}$ converges to

$$
s_{h}+\sum_{k=1}^{n} w_{k}, \quad n \neq 0
$$

as $j \rightarrow \infty$ or $\left\|H_{j}\right\| \rightarrow 0$, where $s_{h}$ is an obvious horizontal section and each $w_{j}$ is a $J_{0}$-holomorphic sphere into a fiber $(M, \omega)$ of $P=\Sigma \times M . "$

The reason is such a convergence result fails in general for two reasons: First unless we specify how the limiting sequence $H_{j}$ converges to 0 , the sequence $s_{j}$ cannot have any limit in any reasonable topology. This is because the case $H=0$ is a singular situation in the study of the Floer moduli space $\mathcal{M}(H, \widetilde{J} ; \widehat{z})$. Secondly even if we specify a good sequence, e.g., consider the 'adiabatic' sequence

$$
H_{1, j}=\epsilon_{j} f_{1}, H_{2, j}=\epsilon_{j} f_{2}, H_{3, j}=\epsilon_{j} f_{3}
$$

for Morse functions $f_{1}, f_{2}, f_{3}$ with the same sequence $\epsilon_{j} \rightarrow 0$, we still have to deal with the degenerate limit, i.e. the limit that contains components of Hausdorff dimension one as studied in section 8 . What we proved in the proof is that we can always produce at least one non-constant $J_{0}$-holomorphic sphere as $j \rightarrow \infty$ without using such a strong convergence result, when the homotopy class of the $s_{j}$ is not trivial (in the fiber direction). 
Remark 10.2. One can easily see that there are many rooms for improvements in the above proof and in the statements of the results in section 9. We summarize several points of improvement:

(1) The statement of Proposition 9.4 can be further improved using more systematic usage of Hamiltonian fibrations and associated Hamiltonian connections in the spirit of [En1] and [Oh9]. (See the proof of Proposition 9.4 above for some flavor thereof.)

(2) All the arguments used in section 9 and 10 can be generalized to the case where $\phi$ is engulfable. This requires a more systematic discussion on the local Floer complexes and more precise estimates involving the curvature of the Hamiltonian fibration.

(3) The inequalities (9.26), (9.27) are obviously not optimal.

In the sequel [Oh10], we will further elaborate the above points and derive various consequences of them in relation to Hofer's geometry of the Hamiltonian diffeomorphism group.

\section{Appendix : Construction of flat connections}

In this appendix, we will construct a flat Hamiltonian connection associated to the triple (8.11)

$$
H_{1}=H, H_{2}=\widetilde{H} \#(\epsilon f), H_{3}=\epsilon f .
$$

We refer to [Oh9] for a detailed calculation of the curvature associated to a deformed mapping cylinder. In our case, such a flat connection in our minimal area metric representation of the fibration $P \rightarrow \Sigma$ can be constructed gluing three twoparameter family

$$
\phi_{i}:(s, t) \in[0,1] \times[0,1] \rightarrow \operatorname{Ham}(M, \omega)
$$

of Hamiltonian diffeomorphisms and then elongating the family in the $s$-direction via a cut-off function $\rho$ of the type (8.2). We now give the explicit formulae of them that glue smoothly along the boundary of $\Sigma_{i}$ according to the gluing rule (8.1). This flat connection has singularities at the two points $p, \bar{p}$ mentioned before. Without loss of any generality, we assume that $H$ is boundary flat, i.e., that there exists $\delta_{0}>0$ such that

$$
H_{t} \equiv 0 ; \quad t \in\left[0, \delta_{0}\right] \cup\left[1-\delta_{0}, 1\right] .
$$

Here come the formulae for $\phi_{i} \mathrm{~s}$ :

$$
\begin{aligned}
& \phi_{1}(s, t)= \begin{cases}i d & 0 \leq t \leq \frac{1-s}{2} \\
\phi_{H}^{\frac{t-\frac{1-s}{2}}{2}} & \frac{1-s}{2} \leq t \leq 1\end{cases} \\
& \phi_{2}(s, t)= \begin{cases}\frac{\frac{1+s}{2}-t}{\frac{1+s}{2}} & 0 \leq t \leq \frac{1-s}{2} \\
\phi_{H}^{\frac{1+s}{2}} & \frac{t-\frac{1-s}{2}}{2} \leq t \leq 1 \\
\phi_{H}^{\frac{1+s}{2}} \circ \phi_{\epsilon f}^{\frac{1+s}{2}} & 1-s\end{cases} \\
& \phi_{3}(s, t)= \begin{cases}\phi_{\epsilon f}^{\frac{t}{2}} & 0 \leq t \leq \frac{1+s}{2} \\
i d & \frac{1+s}{2} \leq t \leq 1 .\end{cases}
\end{aligned}
$$


Compatibility to the gluing rule (8.29) can be achieved by rescaling the domain of $\phi_{i}$ in the $s$-direction in the obvious way, and smoothness of the family away from $p, \bar{p}$ can be checked easily. Flatness of the associated connection obviously follows from the fact that it comes from the above smooth two parameter family of Hamiltonian diffeomorphisms. (See [Ba] for the relevant argument.)

We like to note that due to the boundary flatness assumption (A.1) on $H$ the associated connections converges to the trivial connection on $D^{2}\left(\delta_{0}\right) \backslash\{(0,0)\}$, or equivalently the associated Hamiltonians converges to the zero Hamiltonian on $D^{2}\left(\delta_{0}\right) \backslash\{(0,0)\}$ with respect to the standard metric of $S=\mathbb{R} \times S^{1}$.

Now we elongate the pants in the direction of $s$ using cut-off functions $\rho$ as defined in (8.2), which gives rise to a flat connection on $\Sigma$ that is smooth everywhere except two points $p, \bar{p}$. One important point to note here is that as $\epsilon \rightarrow 0$, the above flat connection on $\Sigma_{1} \cup \Sigma_{2}$ converges to the connection on the mapping cylinder induced by the Hamiltonian $H$ as described in subsection 8.2.

\section{References}

[Ba] Banyaga, A., Sur la structure du groupe des difféomorphismes qui préservent une forme symplectique, Comm. Math. Helv. 53 (1978), 174-227.

[BP] Bialy, M., Polterovich, L., Geodesics of Hofer's metric on the group of Hamiltonian diffeomorphisms, Duke J. Math. 76 (1994), 273-292.

[Ch] Chekanov, Y., Lagrangian intersections, symplectic energy and areas of holomorphic curves, Duke J. Math. 95 (1998), 213-226.

[DS] Dostoglou, D., Salamon, D., Self dual instantons and holomorphic curves, Ann. Math. 139 (1994), 581-640.

[En1] Entov, M., K-area, Hofer metric and geometry of conjugacy classes in Lie groups, Invent. Math. 146 (2001), 93-141.

[En2] Entov, M., Commutator length of symplectomorphisms, Comment. Math. Helv. 79 (2004), 58-104.

[EnP] Entov, M., Polterovich, L., Calabi quasimorphism and quantum homology, Int. Math. Res. Not. no 30 (2003), 1635-1676.

[Fl1] Floer, A., Morse theory for fixed points of symplectic diffeomorphisms, Bull. Amer. Math. Soc. (N.S.) 16 (1987), 279-281.

[F12] Floer, A., The unregularized gradient flow of the symplectic action, Comm. Pure Appl. Math. 43 (1988), 576-611.

[F13] Floer, A., Symplectic fixed points and holomorphic spheres, Commun. Math. Phys. 120 (1989), 575-611.

[FOh] Fukaya, K., Oh, Y.-G., Zero-loop open strings in the cotangent bundle and Morse homotopy, Asian J. Math. 1 (1997), 96-180.

[FOn] Fukaya, K., Ono, K., Arnold conjecture and Gromov-Witten invariants, Topology 38 (1999), 933-1048.

[Gr] Gromov, M., Pseudo-holomorphic curves in symplectic manifolds, Invent. Math. 82 (1985), 307-347.

[GLS] Guillemin, V., Lerman, E., Sternberg, S., Symplectic Fibrations and Multiplicity Diagrams, Cambridge University Press, 1996.

[Ho] Hofer, H., On the topological properties of symplectic maps, Proc. Royal Soc. Edinburgh 115 (1990), 25-38.

[HoS] Hofer, H., Salamon, D., Floer homology and Novikov rings, Floer Memorial Volume (Hofer, H. et al, eds.), Birkhaüser, 1995, pp. 483-524.

[K] Karcher, H., Riemannian center of mass and mollifier smoothing, Comm. Pure Appl. Math. 30 (1977), 509-541.

[LM] Lalonde, F. and McDuff, D., The geometry of symplectic energy, Ann. Math. 141 (1995), 349-371.

[La] Laudenbach, F., Engouffrement symplectique et intersections lagrangiennes, Comment. Math. Helv. 70 (1995), 558-614. 
[LT] Liu, G., Tian, G., Floer homology and Arnold's conjecture, J. Differ. Geom. 49 (1998), $1-74$.

[Mc] McDuff, D., Geometric variants of the Hofer norm, J. Symplectic Geom. 1 (2002), 197-252.

[Mi] Milnor, J., Lectures on the h-Cobordism Theorem, Princeton University Press, Princeton, 1965.

[Oh1] Oh, Y.-G., Removal of boundary singularities of pseudo-holomorphic curves with Lagrangian boundary conditions, Comm. Pure Appl. Math. 45 (1992), 121-139.

[Oh2] Oh, Y.-G., Floer cohomology, spectral sequences, and the Maslov class of Lagrangian embeddings, Internat. Math. Res. Notices 7 (1996), 305-346.

[Oh3] Oh, Y.-G., Symplectic topology as the geometry of action functional, I, Jour. Differ. Geom. 46 (1997), 499-577.

[Oh4] Oh, Y.-G., Symplectic topology as the geometry of action functional, II, Commun. Anal. Geom. 7 (1999), 1-55.

[Oh5] Oh, Y.-G., Chain level Floer theory and Hofer's geometry of the Hamiltonian diffeomorphism group, Asian J. Math. 6 (2002), 579-624, math.SG/0104243; Erratum 7 (2003), 447-448.

[Oh6] Oh, Y.-G., Normalization of the Hamiltonian and the action spectrum, J. Korean Math. Soc. 42 (2005), 65-83.

[Oh7] Oh, Y.-G., Spectral invariants and length minimizing property of Hamiltonian paths, Asian J. Math. (to appear), math.SG/0212337.

[Oh8] Oh, Y.-G., Construction of spectral invariants of Hamiltonian paths on closed symplectic manifolds, "The Breadth of Symplectic and Poisson Geometry ; Festschrift in Honor of Alan Weinstein", Progress in Mathematics, Vol. 232 (Marsden, Jerrold E.; Ratiu, Tudor S., ed.), Birkhäuser, 2004, pp. 525-570.

[Oh9] Oh, Y.-G., An existence theorem, with energy bounds, of Floer's perturbed Cauchy-Riemann equation with jumping discontinuity, preprint, math.SG/0207214.

[Oh10] Oh, Y.-G., Thick and thin decompositions of the Floer moduli spaces and their applications, in preparation.

[Os] Ostrover, Y., A comparison of Hofer's metrics on Hamiltonian diffeomorphisms and Lagrangian submanifolds, Comm. Contemp. Math. 5 (2003), 803-912.

[PSS] Piunikhin, S., Salamon, D., Schwarz, M., Symplectic Floer-Donaldson theory and quantum cohomology, Publ. Newton. Inst. (Thomas, C. B., eds.), vol. 8, Cambridge University Press, Cambridge, England, 1996, pp. 171-200.

[Po1] Polterovich, L., The Geometry of the Group of Symplectic Diffeomorphisms, Birkhäuser, 2001.

[Po2] Polterovich, L., private communication.

[Ra] Rabinowitz, P., Periodic solutions of Hamiltonian systems, Comm. Pure Appl. Math. 31 (1978), 157-184.

[Ru] Ruan, Y., Virtual neighborhood and pseudo-holomorphic curves, Turkish J. Math. 23 (1999), 161-231.

[SU] Sacks, J., Uhlenbeck, K., The existence of minimal immersions of 2 spheres, Ann. Math. 113 (1981), 1-24.

[SZ] Salamon, D., Zehnder, E., Morse theory for periodic solutions of Hamiltonian systems and the Maslov index, Comm. Pure Appl. Math. 45 (1992), 1303-1360.

[Sc] Schwarz, M., On the action spectrum for closed symplectically aspherical manifolds, Pacific J. Math. 193 (2000), 419-461.

[Se] Seidel, P., $\pi_{1}$ of symplectic diffeomorphism groups and invertibles in quantum homology rings, GAFA (1997), 1046-1095.

[St] Strebel, K., Quadratic Differentials, Springer Verlag, Berlin Heidelberg, 1984.

[V] Viterbo, C., Symplectic topology as the geometry of generating functions, Math. Ann. 292 (1992), 685-710.

[Z] Zwiebach, B., Closed string field theory: quantum action and the B-V master equation, Nucl. Phys. B 390 (1993), 33.

Department of Mathematics, University of Wisconsin, Madison, Wi 53706, USA \& Korea Institute for Advanced Study, Seoul, Korea; oh@math.wisc.edu 